\title{
Incretin-based drugs and the incidence of colorectal cancer in patients with type 2 diabetes
}

\author{
Devin Abrahami \\ Department of Experimental Medicine \\ McGill University, Montreal \\ August 2017
}

\begin{abstract}
A thesis submitted to McGill University in partial fulfillment of the requirements of the degree of Master of Science in Experimental Medicine
\end{abstract}

CDevin Abrahami, 2017 


\section{Abstract}

Background: The incretin-based drugs, glucagon-like peptide-1 (GLP-1) analogues and dipeptidylpeptidase-4 (DPP-4) inhibitors, are common second-to-third-line therapies used in the treatment of type 2 diabetes. The safety of these drugs has been controversial; both clinical trials and biological studies have published conflicting evidence on their association with colorectal cancer.

Objectives: To determine whether the use of incretin-based drugs is associated with incident colorectal cancer in patients with type 2 diabetes.

Patients and methods: Using data from the United Kingdom Clinical Practice Research Datalink, we identified a cohort of 112,040 patients newly treated with antidiabetic drugs between January 1, 2007 and March 31, 2015, followed until March 31, 2016. The time-varying use of GLP-1 analogues and DPP-4 inhibitors was compared with the use of sulfonylureas. All exposures were lagged by one year for latency purposes, and to minimize reverse causality and detection bias. Time-dependent Cox proportional hazards models were used to estimate hazard ratios (HRs) and 95\% confidence intervals (Cls) of incident colorectal cancer associated with the use of GLP-1 analogues and DPP-4 inhibitors overall, by cumulative duration of use, and time since initiation. All models were adjusted for a number of important confounders, including age, sex, smoking status, alcohol-related disorders and body mass index.

Results: During 388,619 person-years of follow-up, there were 733 incident colorectal cancer events, generating a crude incidence rate of $1.9(95 \% \mathrm{Cl}: 1.8$ to 2.0$)$ per 1000 person-years. Compared with the use of sulfonylureas, the use of GLP-1 analogues was not associated with 
the incidence of colorectal cancer (2.0 vs 1.6 per 1000 per year, respectively; HR: $1.03 .95 \% \mathrm{Cl}$ : 0.68 to 1.58). Furthermore, there was no evidence of a duration-response relation both in terms of cumulative duration of use ( $\leq 1$ year, HR: 0.68, 95\%: 0.32-1.46; 1.1-2 years, HR: 1.43, 95\% Cl: 0.80 to $2.59 ;>2$ years, $\mathrm{HR}: 1.07,95 \% \mathrm{Cl}: 0.50$ to 2.31 ) and time since initiation ( $\leq 2$ years, HR: $1.22,95 \%$ : 0.62 to $2.39 ;>2$ years, $\mathrm{HR}: 0.96,95 \% \mathrm{Cl}: 0.58-1.59)$. With respect to DPP4 inhibitors, there was no association either overall (1.9 vs 2.1 per 1000 per year, respectively; $\mathrm{HR}: 1.19 .95 \% \mathrm{Cl}: 0.94$ to 1.50 ), by cumulative duration of use ( $\leq 1$ year, $\mathrm{HR}: 1.41,95 \% \mathrm{Cl}: 0.99$ to 2.00 ; $1.1-2$ years, $\mathrm{HR}: 1.00,95 \% \mathrm{Cl}: 0.70$ to 1.44 ; > 2 years, $\mathrm{HR}: 1.20,95 \% \mathrm{Cl}: 0.86$ to 1.67$)$, or by time since initiation ( $\leq 2$ years, HR: $1.34,95 \%$ : 0.96 to $1.86 ;>2$ years, HR: $1.11,95 \% \mathrm{Cl}: 0.85$ to 1.46$)$.

Conclusions: The results of this large population-based study indicate that compared to the use of sulfonylurea, the use of the incretin-based drugs is not associated with the incidence of colorectal cancer among patients with type 2 diabetes. 


\section{Résumé}

Contexte : Les médicaments agissant sur les incrétines, le peptide-1 de type glucagon (GLP-1) et les inhibiteurs de la dipeptidyllpeptidase 4 (DPP-4) sont des traitements de deuxième et troisième intentions courants utilisés dans le traitement du diabète de type 2 . L'innocuité de ces médicaments est controversée; les études cliniques et les études biologiques ont mené à la publication de preuves contradictoires sur leur lien avec le cancer colorectal.

Objectifs : Déterminer si l'utilisation de médicaments agissant sur les incrétines est associée à l'apparition d'un cancer colorectal chez des patients atteints de diabète de type 2.

Patients et méthodes : À l'aide des données du Clinical Practice Research Datalink du RoyaumeUni, nous avons identifié une cohorte de 112040 patients qui ont reçu un traitement à l'aide d'antidiabétiques entre le $1^{\text {er }}$ janvier 2007 et le 31 mars 2015, et qui ont été suivis jusqu'au 31 mars 2016. L'utilisation variable dans le temps d'analogues du GLP-1 et d'inhibiteurs de la DPP4 a été comparée à l'utilisation des sulfonylurées. Toutes les expositions ont été décalées d'une année comme période de latence et pour minimiser le biais de détection et de causalité inverse. Les modèles à risques proportionnels de Cox liés au temps ont été utilisés pour estimer les rapports de risque (RR) et les intervalles de confiance (IC) à $95 \%$ portant sur l'apparition du cancer colorectal associée globalement à l'utilisation d'analogues du GLP-1 et d'inhibiteurs de la DPP-4, en fonction de la durée cumulée d'utilisation et du temps écoulé depuis le début du traitement. Tous les modèles ont été ajustés pour un certain nombre de variables confusionnelles importantes, y compris l'âge, le sexe, le tabagisme, les problèmes liés à la consommation d'alcool et l'indice de masse corporelle. 
Résultats : Au cours d’une période de 388619 années-personnes de suivi, il y a eu 733 événements d'apparition de cancer colorectal se traduisant par un taux d'incidence brut de 1,9 (IC $95 \%$ : 1,8 à 2,0) par 1000 années-personnes. Par rapport à l'utilisation des sulfonylurées, l'utilisation d'analogues du GLP-1 n'était pas associée à l'apparition de cancer colorectal (2,0 vs 1,6 pour 1000 par an, respectivement; RR : 1,03; IC $95 \%$ :0,68 à 1,58). Par ailleurs, aucune preuve ne démontrait qu'il existait un lien durée-effet à la fois en termes de durée cumulée d'utilisation ( $\leq 1$ année, RR : 0,68; IC $95 \%: 0,32$ à 1,46; 1,1 à 2 ans, RR : 1,43; IC $95 \%: 0,80$ à 2,59; > 2 ans, RR : 1,07; IC $95 \%: 0,50$ à 2,31) et de temps écoulé depuis le début du traitement ( $\leq 2$ ans, RR : 1,22; IC $95 \%: 0,62$ à 2,39; > 2 ans, RR : 0,96; IC $95 \%: 0,58-1,59)$. En ce qui concerne les inhibiteurs de la DPP-4, il n'y avait aucun lien, que ce soit globalement (1,9 vs 2,1 pour 1000 par an, respectivement; RR : 1,19; IC $95 \%$ : 0,94 à 1,50), en fonction de la durée cumulée d'utilisation ( $\leq 1$ année, RR : 1,41; IC $95 \%$ : 0,99 à 2,00; 1,1 à 2 ans, RR : 1,00; IC $95 \%$ : 0,70 à 1,44; > 2 ans, RR : 1,20; IC $95 \%$ : 0,86 à 1,67) ou en fonction du temps écoulé depuis le début du traitement ( $\leq 2$ ans, RR : 1,34; IC $95 \%: 0,96$ à 1,86; > 2 ans, RR : 1,11; IC $95 \%: 0,85$ à $1,46)$.

Conclusions : Les résultats de cette vaste étude populationnelle indiquent que, par rapport à l'utilisation des sulfonylurées, l'utilisation des médicaments agissant sur les incrétines n'était pas associée à l'apparition du cancer colorectal chez les patients atteints de diabète de type 2 . 


\section{Acknowledgements}

With the utmost gratitude, I would like to thank my two supervisors, Dr. Laurent Azoulay and Dr. Michael Pollak. Through the support and guidance of Dr. Azoulay, I have realized a passion for pharmacoepidemiology and advancing research. He has motivated me to think critically, become a better researcher and set high expectations of myself. Thank you for taking on a mentorship role far exceeding the responsibilities of a traditional supervisor. Dr. Pollak, thank you for providing me with your expertise on cancer. You offered interesting perspectives on the biology of cancer and mechanistic roles that allowed me to better shape my thesis and broaden my overall understanding of the field. I feel very fortunate to have had two dedicated supervisors. Hui Yin, thank you for all your statistical expertise. Not only did you construct the cohort and assist with the analysis, but you happily answered any questions I had about statistics and modelling. The patience you showed me was truly appreciated. Dr. Oriana Yu, thank you for your clinical expertise on diabetes and the unique insights you provided during analysis. You have all contributing to an enriching and positive experience, and for that I thank you. 


\section{Contribution of authors}

Devin Abrahami drafted the thesis and corresponding manuscript. All authors contributed to the study concept and design. Devin Abrahami, Hui Yin and Dr. Laurent Azoulay contributed to the statistical analyses. Drs. Michael Pollak and Oriana Yu contributed to the interpretation of results and provided expertise on the biologic plausibility. All authors critically revised the manuscript for important intellectual content. Dr. Azoulay acquired the data, supervised the study and is the guarantor. 


\section{Table of contents}

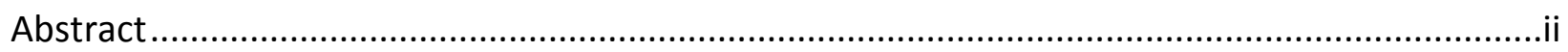

Résumé

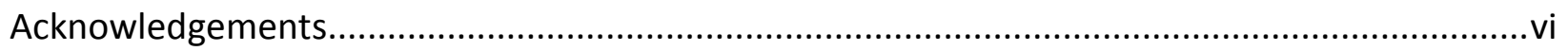

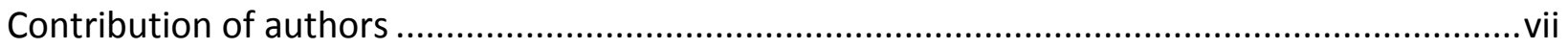

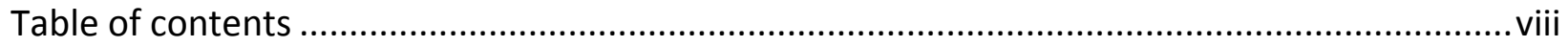

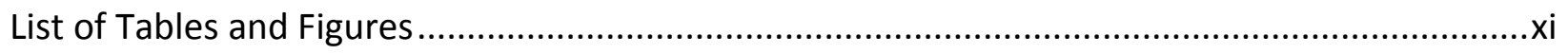

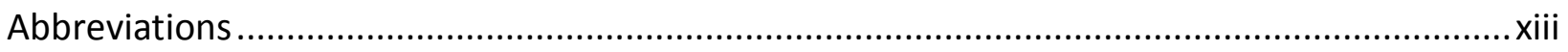

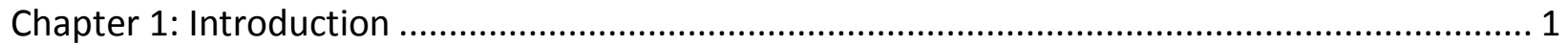

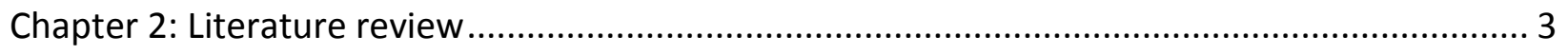

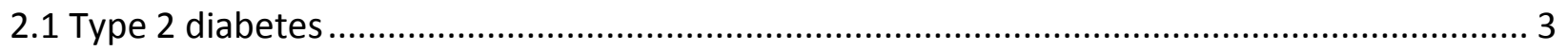

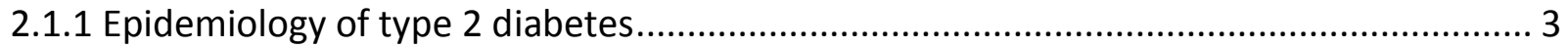

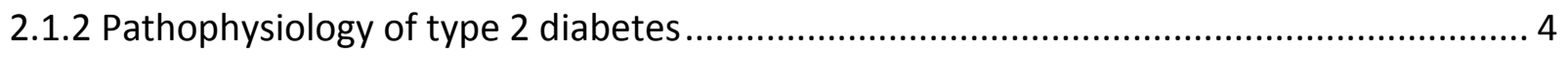

2.1.3 Clinical management of type 2 diabetes............................................................. 5

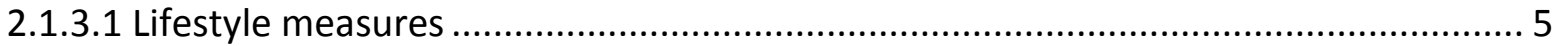

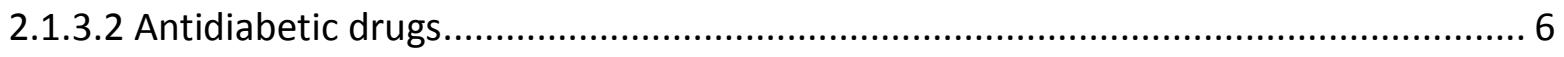

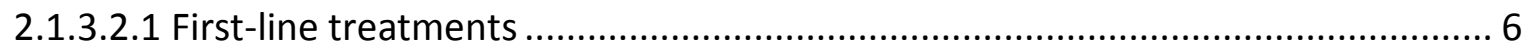

2.1.3.2.2 Second-to-third-line treatments............................................................ 7

2.1.3.2.3 Last-line treatment ....................................................................... 11

2.2 Association between type 2 diabetes and cancer incidence ......................................... 13

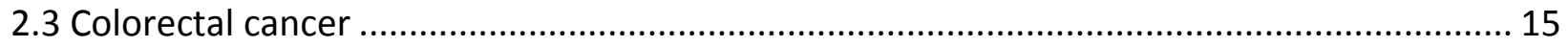

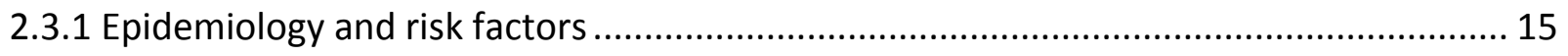

2.3.2 Association between type 2 diabetes and colorectal cancer .................................... 16

2.4 Colorectal cancer and the incretin-based drugs ........................................................... 18

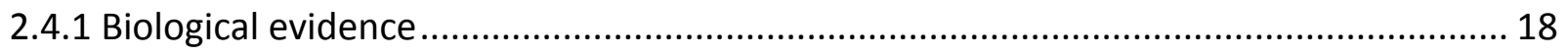

2.4.1.1 Glucagon-like peptide-1 analogues ................................................................. 18

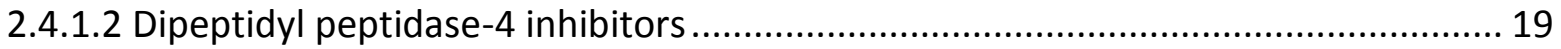

2.4.2 Safety reviews: United States Food and Drug Administration and European Medicines

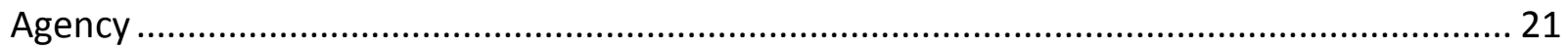




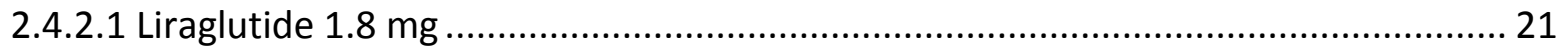

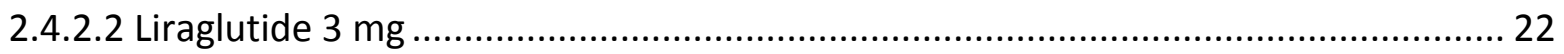

2.4.3 Post-marketing randomized controlled trials .......................................................... 23

2.4.3.1 Glucagon-like peptide-1 analogues ........................................................................... 24

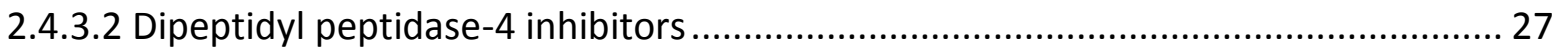

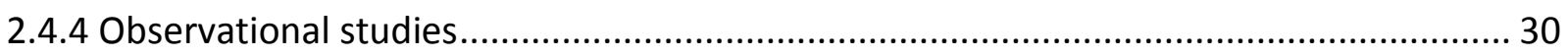

Chapter 3: Objectives and hypotheses .............................................................................. 33

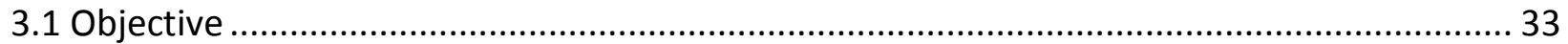

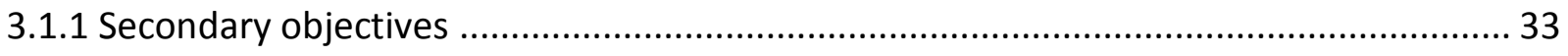

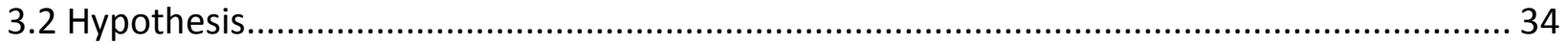

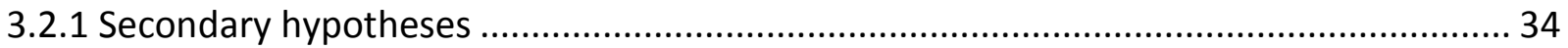

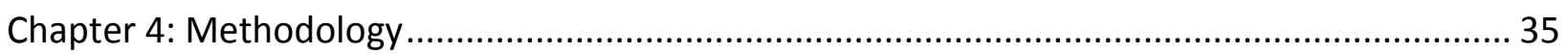

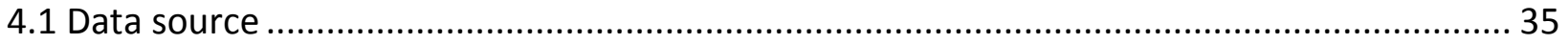

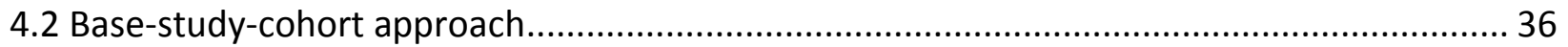

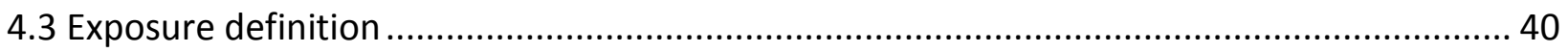

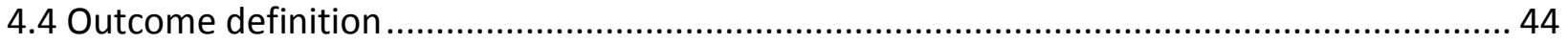

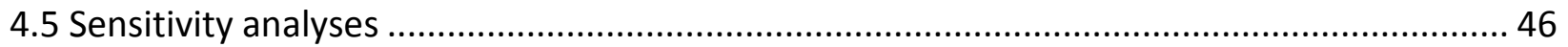

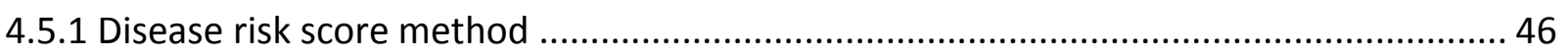

4.5.2 Marginal structural modelling ..................................................................................... 46

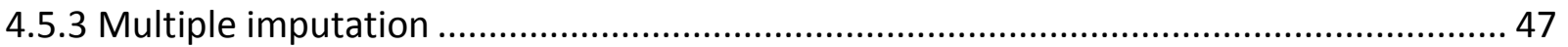

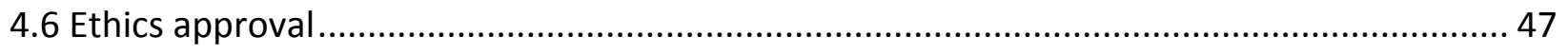

Chapter 5: Incretin-based drugs and the incidence of colorectal cancer in patients with type 2 diabetes: population-based cohort study .............................................................................. 48

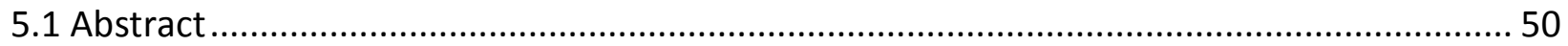

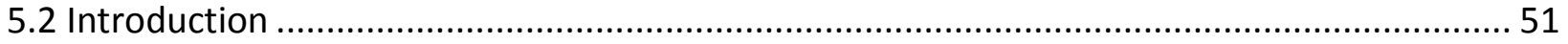

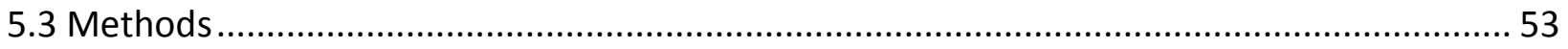

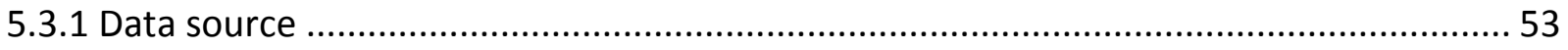

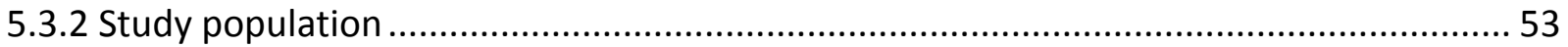

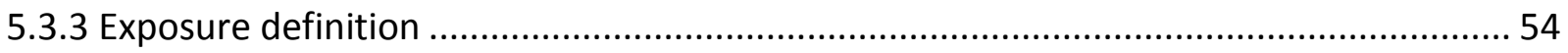

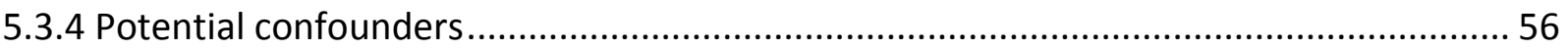

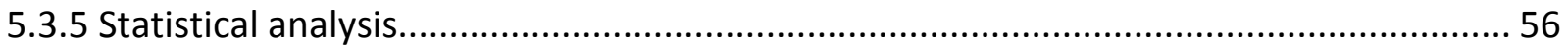




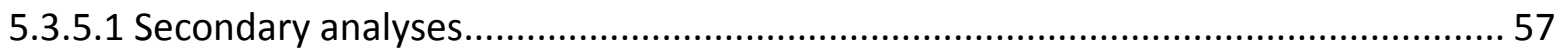

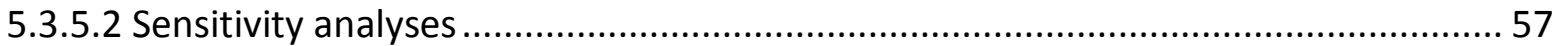

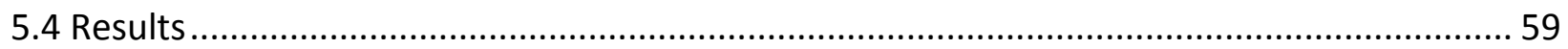

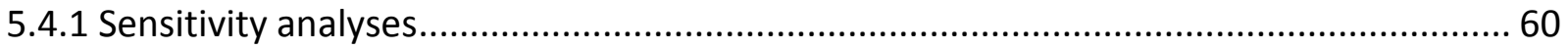

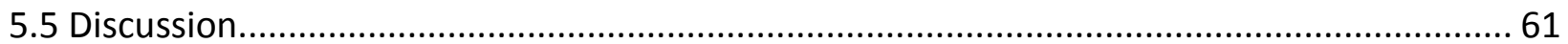

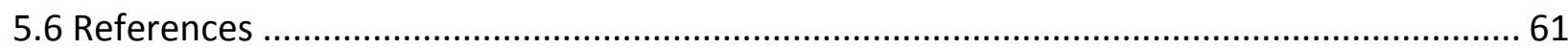

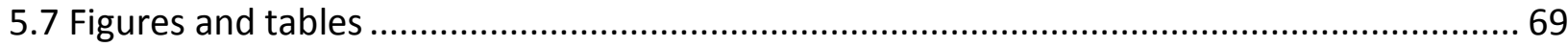

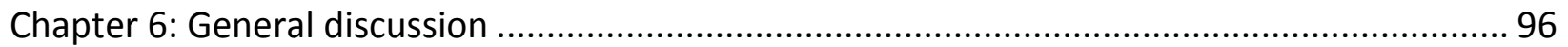

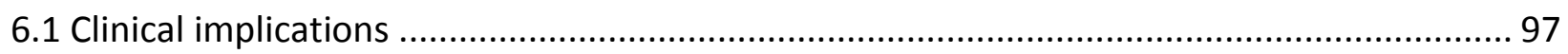

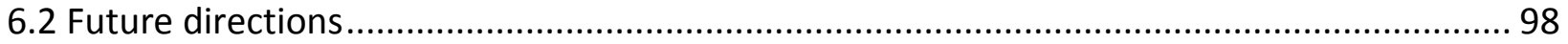

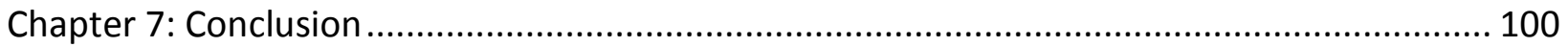

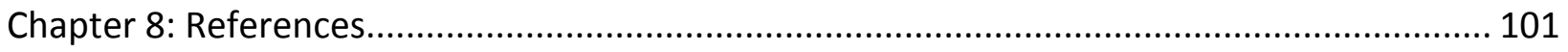




\section{List of Tables and Figures}

\section{Figures}

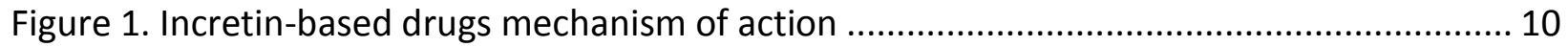

Figure 2. Forest plot: type 2 diabetes and cancer incidence ............................................. 14

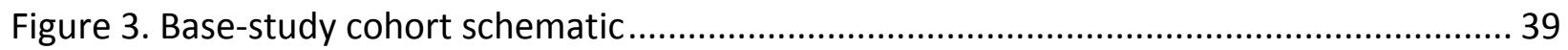

Figure 4. Exposure definition: single drug exposure ......................................................... 41

Figure 5. Exposure definition: multiple drug exposures......................................................... 43

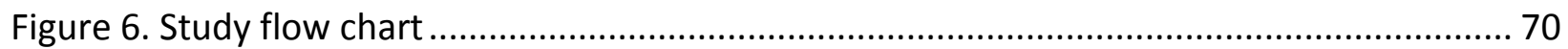

\section{Tables}

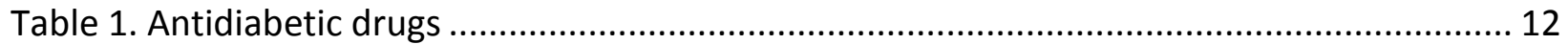

Table 2. Post-marketing randomized controlled trials: glucagon-like peptide-1 analogues........ 27

Table 3. Post-marketing randomized controlled trials: dipeptidyl peptidase-4 inhibitors .......... 30

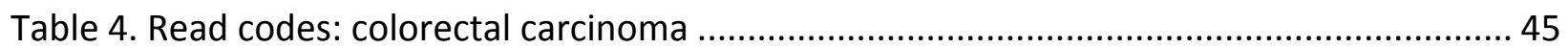

Table 5. Baseline Demographic and Clinical Characteristics of the Cohort and Stratified by Drug Use at Cohort Entry................................................................................................ 71

Table 6. Crude and Adjusted HRs for the Association Between the Use of GLP-1 Analogues and

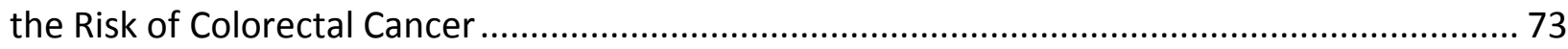

Table 7. Crude and Adjusted HRs for the Association Between the Use of DPP-4 Inhibitors and the Risk of Colorectal Cancer .................................................................................................... 74

\section{Supplementary Figures}

Supplementary Figure 1. Hierarchical exposure definition ................................................... 76

Supplementary Figure 2. Forest plot of primary and sensitivity analyses ............................. 77

\section{Supplementary Tables}

Supplementary Table 1. Crude and Adjusted HRs for the Association Between the Use of GLP-1 Analogues and the Risk of Colorectal Cancer (GLP-1 Type) ..................................................... 78 Supplementary Table 2. Crude and Adjusted HRs for the Association Between the Use of DPP-4 Inhibitors and the Risk of Colorectal Cancer (DPP-4 Type)................................................... 79 Supplementary Table 3. Crude and Adjusted HRs for the Association Between the Use of GLP-1 Analogues and the Risk of Colorectal Cancer (Colon Cancer and Rectal Cancer Specifically) ..... 80 Supplementary Table 4. Crude and Adjusted HRs for the Association Between the Use of DPP-4 Inhibitors and the Risk of Colorectal Cancer (Colon Cancer and Rectal Cancer Specifically) ....... 81 
Supplementary Table 5. Crude and Adjusted HRs for the Association Between the Use of GLP-1 Analogues and the Risk of Colorectal Cancer (2-year Lag Period)........................................... 82 Supplementary Table 6. Crude and Adjusted HRs for the Association Between the Use of DPP-4 Inhibitors and the Risk of Colorectal Cancer (2-year Lag Period). 83 Supplementary Table 7. Crude and Adjusted HRs for the Association Between the Use of GLP-1 Analogues and the Risk of Colorectal Cancer (No Lag Period) .................................................. 84 Supplementary Table 8. Crude and Adjusted HRs for the Association Between the Use of DPP-4 Inhibitors and the Risk of Colorectal Cancer (No Lag Period). 85 Supplementary Table 9. Crude and Adjusted HRs for the Association Between the Use of GLP-1 Analogues and the Risk of Colorectal Cancer (Stratified by Screening) ................................... 86 Supplementary Table 10. Crude and Adjusted HRs for the Association Between the Use of DPP-4 Inhibitors and the Risk of Colorectal Cancer (Stratified by Screening) 87 Supplementary Table 11. Crude and Adjusted HRs for the Association Between the Use of GLP-1 Analogues and the Risk of Malignant Colorectal Cancer 88 Supplementary Table 12. Crude and Adjusted HRs for the Association Between the Use of DPP-4 Inhibitors and the Risk of Malignant Colorectal Cancer 89 Supplementary Table 13. Crude and Adjusted HRs for the Association Between the Use of GLP-1 Analogues and the Risk of Colorectal Cancer (Stratified by Disease Risk Score) ....................... 90 Supplementary Table 14. Crude and Adjusted HRs for the Association Between the Use of DPP-4 Inhibitors and the Risk of Colorectal Cancer (Stratified by Disease Risk Score)..... 91 Supplementary Table 15. Crude and Adjusted HRs for the Association Between the Use of GLP-1 Analogues and the Risk of Colorectal Cancer (Marginal Structure Model) 92 Supplementary Table 16. Crude and Adjusted HRs for the Association Between the Use of DPP-4 Inhibitors and the Risk of Colorectal Cancer (Marginal Structural Model) 93 Supplementary Table 17. Crude and Adjusted HRs for the Association Between the Use of GLP-1 Analogues and the Risk of Colorectal Cancer (Multiple imputation) ...................................... 94 Supplementary Table 18. Crude and Adjusted HRs for the Association Between the Use of DPP-4 Inhibitors and the Risk of Colorectal Cancer (Multiple imputation)...... 95 


\section{Abbreviations}

\begin{tabular}{|c|c|}
\hline AMP & Adenosine monophosphate \\
\hline ATP & Adenosine triphosphate \\
\hline BMI & Body mass index \\
\hline $\mathrm{Cl}$ & Confidence interval \\
\hline CPRD & Clinical practice research datalink \\
\hline DPP-4 & Dipeptidyl peptidase-4 \\
\hline DRS & Disease risk score \\
\hline EMA & European Medicines Agency \\
\hline ERK1/2 & Extracellular signal-related kinase \\
\hline FAP & Fibroblast activation protein \\
\hline FDA & Food and Drug Administration \\
\hline FGF7 & Fibroblast growth factor 7 \\
\hline FPG & Fasting plasma glucose \\
\hline GLP-1 & Glucagon-like peptide-1 \\
\hline GSK3 & Glycogen synthase kinase 3 \\
\hline $\mathrm{HbA1c}$ & Glycosylated hemoglobin level \\
\hline HR & Hazard ratio \\
\hline IPCWs & Inverse probability of censoring weights \\
\hline IPTWs & Inverse-probability-of-treatment weights \\
\hline OGTT & Oral glucose tolerance test \\
\hline RCTs & Randomized controlled trials \\
\hline SD & Standard deviation \\
\hline SGLT2 & Sodium-glucose co-transporter 2 \\
\hline TZD & Thiazolidinedione \\
\hline UK & United Kingdom \\
\hline US & Unites States \\
\hline
\end{tabular}




\section{Chapter 1: Introduction}

Type 2 diabetes is a common metabolic disorder affecting $9.3 \%$ of Canadians ${ }^{1}$ and 1 in 11 adults worldwide. ${ }^{2}$ In addition to the micro- and macro-vascular complications of this disease, ${ }^{34}$ type 2 diabetes has been associated with an increased risk of several cancers ${ }^{5-14}-a$ phenomenon that has been studied since the late $1800 \mathrm{~s} .{ }^{15}$ Indeed, there is considerable evidence that type 2 diabetes is associated with an increased risk of colorectal, breast, bladder, liver, endometrium, pancreas, kidney cancers, ${ }^{5-12}$ while being inversely associated with prostate cancer. ${ }^{1314}$ The relationship between type 2 diabetes and the incidence of cancer is not entirely understood, but may be explained by the mitogenic effects of insulin, ${ }^{510}$ and/or by shared common risk factors such as obesity, diet and smoking. ${ }^{7} 1012$

Over the years, there has been an increased interest in determining whether antidiabetic drugs could influence the incidence of cancer in patients with type 2 diabetes. For example, the use of metformin has been shown to be associated with a decreased risk of several cancers in several observational studies, including cancers of the breast, ${ }^{16}$ pancreatic, ${ }^{17}$ liver, ${ }^{17} 18$ lung, ${ }^{18}$ and colorectal. ${ }^{18}$ However, as previously reviewed, the majority of these studies had important methodological shortcomings that likely exaggerated metformin's purported benefits. ${ }^{19}$ The evidence on other antidiabetic drugs is also mixed; use of sulfonylureas has been associated with both increased and decreased overall cancer incidence, ${ }^{2021}$ as has the use of thiazolidinediones (TZDs). ${ }^{22-24}$ Finally, insulins such as the longacting analogue, glargine, has been associated with an increased risk of breast cancer. ${ }^{25}$ 
Given this backdrop, there is a need to assess whether newly-marketed drugs could also influence the incidence of cancer in patients with type 2 diabetes. This includes incretin-based drugs, a group of drugs that have stirred controversy soon after their introduction on the market. ${ }^{26}$ Indeed, there were initial concerns that these drugs may be associated with an increased risk of pancreatic cancer, ${ }^{27} 28$ although these were not corroborated in subsequent large observational studies. ${ }^{29}$ Recently, new safety concerns have emerged from randomized controlled trials (RCTs) with respect to other malignancies, such as breast ${ }^{30}$ and colorectal cancer. ${ }^{31-34}$ With respect to the latter, only one observational study has been conducted to assess this association. ${ }^{35}$ While that study did not report an increased risk of colorectal cancer with incretin-based drugs, it had certain methodological shortcomings such as short duration of follow-up. ${ }^{35}$ Thus, given the limited evidence on this safety question, there is an important need to determine whether the use of incretin-based drugs is associated with an increased risk of colorectal cancer in the natural setting of clinical practice. Such findings would be of interest to physicians, patients, and regulatory agencies, and may impact clinical practice and the management of type 2 diabetes. 


\section{Chapter 2: Literature review}

The following chapter is divided into four sections. The first section provides an overview of type 2 diabetes, including its epidemiology, pathophysiology, and different treatment options. The second section describes the association between type 2 diabetes and cancer incidence. The third section provides background information on colorectal cancer and describes its association with type 2 diabetes. Finally, the last section details what is known on the association between incretin-based drugs and colorectal cancer, reviewing previous research on this association.

\subsection{Type 2 diabetes}

\subsubsection{Epidemiology of type 2 diabetes}

According to the international diabetes federation, diabetes affects more than $\mathbf{4 0 0}$ million people globally, whereby $90 \%$ of these diagnoses are type 2 diabetes. ${ }^{2}$ As of 2016 , there were 11 million people living with diabetes and prediabetes in Canada, with approximately 3.5 million prevalent cases. ${ }^{1}$ Type 2 diabetes is considered one of the fastest growing diseases in Canada, and has a yearly incidence of 60,000 cases per year. ${ }^{36}$ As a highly prevalent disease, it is associated with an economic burden of 16.9 billion dollars per year. ${ }^{37}$ Though the exact cause of type 2 diabetes is unknown, it is thought to be a combination of genetic and lifestyle factors; major risk factors include obesity, sedentary behaviour, and smoking. ${ }^{38}$ Type 2 diabetes is associated with several negative consequences, include macro-vascular complications (including stroke, heart failure and coronaropathy) ${ }^{3}$ and micro-vascular complications 
(nephropathy, retinopathy, and neuropathy). ${ }^{4}$ These chronic complications may contribute to an increase in premature death, as well as a decrease in the quality of life among those with type 2 diabetes. ${ }^{39}$

\subsubsection{Pathophysiology of type 2 diabetes}

Type 2 diabetes is a metabolic disorder characterised by two defects. ${ }^{4041}$ The first is increased insulin resistance, whereby the traditional insulin-target tissues (muscle, fat and liver) fail to respond adequately to normal insulin levels over time. ${ }^{42}$ The second is beta cell dysfunction, resulting in decreased insulin secretion from the pancreatic beta cells. ${ }^{42}$ Initially, pancreatic beta cells compensate in response to tissue insulin resistance by producing elevated amounts of insulin. ${ }^{43}$ Eventually, this compensation fails, leading to a persistent state of hyperglycemia and a diagnosis of type 2 diabetes. ${ }^{40-43}$

According to the American Diabetic Association 1997 guidelines and the World Health Organization (WHO) National diabetic group of 2006, type 2 diabetes is diagnosed after an elevated glucose reading accompanying symptoms of the disease (polyuria, polydipsia, polyphagia and weight loss), or by two elevated glucose readings on separate occasions without symptoms ${ }^{44}$ The laboratory reading may be in terms of fasting plasma glucose (FPG), with abnormal levels above $126 \mathrm{mg} / \mathrm{dL}$, or with an oral glucose tolerance test (OGTT), whereby abnormal levels are above $200 \mathrm{mg} / \mathrm{dL}$ two hours after a $75 \mathrm{~g}$ dose of glucose. ${ }^{45} \mathrm{As}$ of 2009 , an additional diagnostic criterion was added, in that a glycosylated hemoglobin level (HbA1c) above $6.5 \%$ is indicative of diabetes. ${ }^{45}$ Testing on HbA1c levels is considered practical, as 
individuals are not required to fast as in FPG and OGTT. ${ }^{45} 46$ Finally, $\mathrm{HbA} 1 \mathrm{c}$ levels provide a history of glycaemia over the prior 3 months, rather than at a single point in time. ${ }^{46}$

\subsubsection{Clinical management of type 2 diabetes}

Type 2 diabetes is a highly dynamic disease with many available treatment options. Typical management of this disease includes lifestyle measures as well as pharmacological drugs. The specific course of treatment is a per patient decision, and depends on personal risk factors, family history, and presence or absence of diabetic complications. ${ }^{47}$

\subsubsection{Lifestyle measures}

One of the most significant risk factors for type 2 diabetes is obesity and a sedentary lifestyle. ${ }^{38}$ As such, the first-line of treatment for type 2 diabetes is diet and exercise. ${ }^{44} 47$ Exercise has been shown to increase insulin sensitivity and improve glycemic control. ${ }^{47}$ Other lifestyle modifications may prevent the progression and development of type 2 diabetes, such as limiting alcohol intake, abstaining from smoking and maintaining a healthy body mass index (BMI) below $25 \mathrm{~kg} / \mathrm{m}^{2}$ through a proper diet. ${ }^{44} 4849$ When lifestyle modifications no longer adequately control blood glucose levels, several antidiabetic drugs may be used as treatment; either alone or in combination with other glucose-lowering drugs. 


\subsubsection{Antidiabetic drugs}

There are many different classes of antidiabetic drugs (Table 1). This section will provide details on all available drugs and their mechanisms of action. Type 2 diabetes is a highly dynamic disease, requiring constant monitoring of patient care. If $\mathrm{HbA1c}$ levels remain above target for 3 months, a new drug is prescribed either alone or in combination with the previous antidiabetic drug. ${ }^{42}$

\subsection{First-line treatments}

The biguanide drug class, including phenformin, buformin and metformin, was first introduced in Europe in 1959, followed by approval in Canada in $1972 .{ }^{50}$ Use of metformin quickly supplanted phenformin and buformin, as the latter drugs were removed from the market due to associations with lactic acidosis. ${ }^{50} 51$ As a result of this controversy, the US food and drug administration (FDA) was hesitant to approve the biguanide class; metformin was approved for use in 1995. ${ }^{51}$ This approval coincided with results from the United Kingdom (UK) Prospective Diabetes Study, an RCT from 1977-1997 which showed that metformin was superior to sulfonylureas and insulin in preventing hypoglycemic attacks and diabetes-related complications. ${ }^{52}$ Since then, metformin has been widely accepted as the first-line treatment for type 2 diabetes. ${ }^{42} 474952$ Metformin is also indicated for polycystic ovary syndrome. ${ }^{51}$

Metformin exerts its antidiabetic effects by supressing hepatic glucose production and decreasing insulin resistance. ${ }^{49}$ Other mechanisms of action include fatty acid oxidation, activation of adenosine monophosphate (AMP)-activated protein kinase and decreased absorption of glucose from the gastrointestinal tract. ${ }^{44}$ As the biguanide drug class does not 
directly stimulate insulin release, these drugs are associated with a low risk of hypoglycemia. ${ }^{44}$

49 Metformin is generally considered to be well tolerated, and may be associated with weight loss in obese patients. ${ }^{47}$ The most common side effects are gastrointestinal problems, and they may cause lactic acidosis in patients with renal disease. ${ }^{44} 4749$ However, the incidence of lactic acidosis among users of metformin is less than 1 per 10,000 person-years, which is 10 to 20 times less than that of the other biguanide drugs. ${ }^{51}$ As such, metformin can be used as a firstline treatment to safely lower $\mathrm{HbA} 1 \mathrm{c}$ levels by $1-2 \% .^{53}$

\subsection{Second-to-third-line treatments}

The first-generation sulfonylureas were approved in 1956 in Europe. ${ }^{53}$ In 1984, more potent second-generation sulfonylureas were approved for use in the US. ${ }^{53}$ Sulfonylureas are insulin secretagogues, and act by binding to potassium adenosine triphosphate (ATP) channels in the beta cell plasma membrane. ${ }^{44} 49$ Through binding, potassium channels close and calcium channels open, allowing cytoplasmic calcium levels to rise which stimulate insulin release. ${ }^{49}$ These drugs have been shown to effectively reduce $\mathrm{HbA1c}$ levels by $1-2 \% .{ }^{53}$ However, as sulfonylureas directly stimulate insulin secretion in a glucose-independent fashion, they are associated with an increased risk of hypoglycemia. ${ }^{44} 4749$ These drugs have also been associated with an increased risk of cardiovascular events and mortality. ${ }^{54}$

First approved in the Unites States in 1997, meglitinides are non-sulfonylurea insulin secretagogues that reduce $\mathrm{HbA} 1 \mathrm{c}$ levels by $1-1.5 \% .{ }^{53}$ Compared to sulfonylureas, meglitinides bind to a different binding site on the potassium ATP channel with a weaker affinity, leading to a shorter half-life and lower risk of hypoglacemia. ${ }^{44}$ These drugs are not commonly prescribed, 
but may be used for those with irregular eating habits or those with late postprandial hypoglycemia when prescribed sulfonylureas. ${ }^{47}$ Though the risk of hypoglycemia is lower than that of sulfonylureas, it remains the most common adverse event. ${ }^{53}$

$\alpha$-glucosidase inhibitors are an oral class of antidiabetic drugs first approved by the FDA in $1995 .{ }^{53}$ They exert their effects by inhibiting the $\alpha$-glucosidase enzyme in the small intestine.$^{53}$ This delays carbohydrate absorption, which minimizes the rise in blood glucose level after a meal, effectively reducing $\mathrm{HbA} 1 \mathrm{c}$ levels by $0.5 \% .{ }^{44}$ These drugs are frequently associated with gastrointestinal side effects, and thus they are not commonly prescribed..$^{39}$

A highly controversial class is the TZD class. Troglitazone was the first drug in this class to be approved by the FDA in 1997, followed by pioglitazone and rosiglitazone in $1999 .{ }^{53}$ These insulin sensitizers exert their effects by binding to the peroxisome proliferator activator receptor- $\gamma$ and activating it, thus increasing glucose uptake of adipose, muscle and liver tissues. ${ }^{47}$ TZDs were recommended for use in those with renal impairments, were well tolerated in older adults compared to metformin ${ }^{44}$ and were found to lower HbA1c levels by 1 $1.5 \% .{ }^{53}$

Every individual TZD drug has been associated with a different controversy. In 2000, troglitazone was withdrawn from the market after being found to cause liver damage..$^{53}$ The other TZDs were used effectively for over a decade, but are no longer commonly prescribed. In 2010, the FDA restricted the use of rosiglitazone, ${ }^{53}$ as it was shown to be associated with adverse cardiovascular events. ${ }^{55}$ The third drug in this class, pioglitazone, was found to be associated with bladder cancer, and thus is limited in its use. ${ }^{24}$ Due to their associated controversies, there is a gap in the market left by the decreased use of TZDs. Patients who are 
not well managed on metformin or sulfonylurea, but do not require the use of insulin, may benefit from newer second-to-third-line treatments.

The incretin-based drug class, which is comprised of glucagon-like peptide-1 (GLP-1) analogues and dipeptidyl peptidase-4 (DPP-4) inhibitors, are a newer group of antidiabetic drugs, first approved in the United States (US) and the UK in 2005 and 2007, respectively. ${ }^{53} 56$ These drugs take advantage of the incretin effect, whereby oral glucose stimulates more insulin release than intravenous glucose. ${ }^{4757}$ In patients with type 2 diabetes, this effect is lost. ${ }^{4757}$ Thus, the incretin-based drugs are used to improve glycemic control by targeting this system; GLP-1 analogues effectively reduce HbA1c levels by $1 \%$, while DPP-4 inhibitors decrease levels by $0.8 \% .^{53}$

The mechanism of action of the incretin-based drugs is illustrated in Figure 1 (reprinted with permission from the British Journal of Cardiology). ${ }^{58} \mathrm{GLP}-1$ analogues are injectable drugs that reversibly bind to the GLP-1 receptor to activate it, triggering insulin release from pancreatic beta cells after glucose tngestion. ${ }^{56}$ DPP-4 inhibitors are oral drugs that act by inhibiting the DPP-4 enzyme, which is normally responsible for the rapid degradation of the incretin hormones. ${ }^{56}$ Once inactive, endogenous levels of incretin hormones rise, and are readily available to stimulate insulin secretion glucose dependently. ${ }^{5657}$ This glucose-dependent mechanism accounts for their lower risk of hypoglycemia, compared with other antidiabetic drugs. ${ }^{475657}$ Finally, these drugs are commonly prescribed due to their favourable effects on body weight; GLP-1 analogues have been shown to have weight lowering effects, while DPP-4 inhibitors are weight neutral. ${ }^{5657}$ The incretin-based drugs are effective and have favourable side effects, but they are not without controversy. When the drugs were first released, they 
were reports of acute pancreatitis, ${ }^{27} 28$ and have more recently been associated with certain malignancies (pancreas and breast). ${ }^{29} 3059$

\section{Figure 1. Incretin-based drugs mechanism of action}

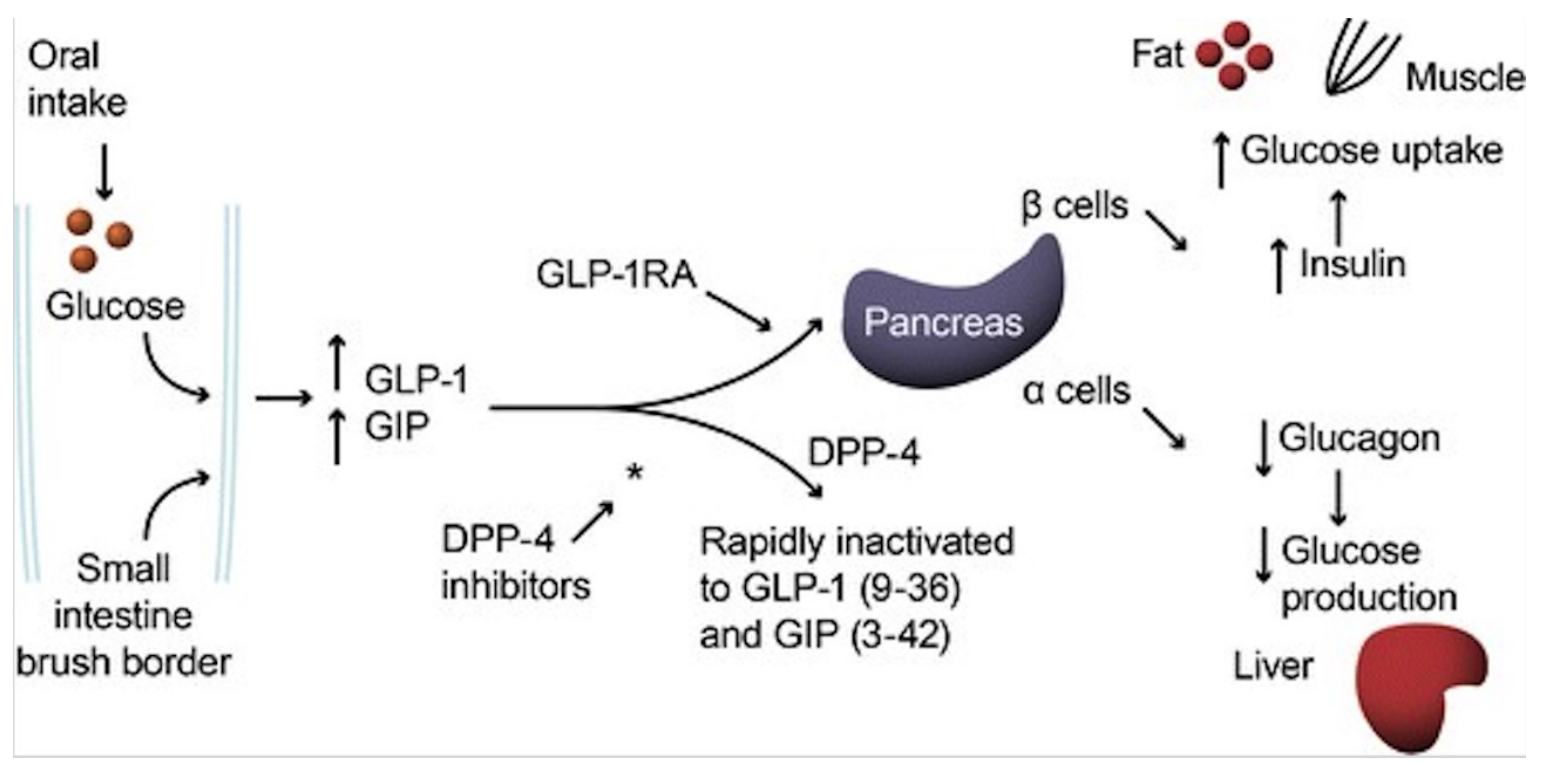

Reprinted with permission from the British Journal of Cardiology ${ }^{58}$

The newest class of antidiabetic drugs are sodium-glucose co-transporter 2 (SGLT2) inhibitors, first approved in Europe in 2012 and the Unites States in $2013 .{ }^{60}$ These drugs increase urinary glucose excretion by blocking glucose reabsorption in the proximal renal tubule. ${ }^{47}$ As these drugs lower glucose insulin independently, they may be used in advanced stage of disease when pancreatic beta cell function is lost. ${ }^{47}$ These drugs effectively lower $\mathrm{HbA} 1 \mathrm{c}$ levels by $0.5-0.8 \%$, and show promising potential for the future of diabetes care. ${ }^{60}$ However, the long-term effects of these drugs are unknown, and cannot be effectively studied at this time. Currently, the most common side effect associated with the use of SGLT2 inhibitors are urinary tract infections.$^{47}$ However, there are increasing concerns that some drugs of this 
class may cause ketoacidosis and lower limb amputations. ${ }^{61-63}$ Long-term RCT data and observational studies are needed to further study the safety of these drugs.

\subsection{Last-line treatment}

Insulin therapies are typically used in patients with severe hyperglycemia, or when oral antidiabetic drugs fail to maintain adequate glucose control. ${ }^{49}$ Patients may be treated with basal insulin, a long-acting insulin that controls blood glucose between meals, if some beta cell function remains. ${ }^{44}$ Patients with impaired beta cell function may require bolus insulin, a more rapid-action dose taken before meals. ${ }^{44} \mathrm{~A}$ well-known adverse event associated with insulin therapy is hypoglycemia; a condition that is more frequently associated with bolus insulin as it has a shorter duration of action than basal insulin. ${ }^{44}$

Insulin analogues were developed as an alternative to traditional insulin therapies to lower the incidence of hypoglacemia. ${ }^{64}$ The first short-acting insulin analogue (lispro) was approved by the FDA in 1996, followed by the approval of long-acting insulin analogues (glargine) in $2000 .{ }^{64}$ Short-acting analogues begin to work 15 minutes after injection and are quickly absorbed, while long-acting drugs can maintain basal insulin levels for about 24 hours. ${ }^{65}$ Long-acting analogues have a lower risk of hypoglycemia, but this class as a whole is not without risk. Treatment with any type of insulin is associated with a risk of hypoglycemia, which is a risk factor for cardiovascular disease. ${ }^{49}$ Finally, these therapies may cause weight gain in obese patients. ${ }^{49}$ 
Table 1. Antidiabetic drugs

\begin{tabular}{lcc}
\hline Class & Mechanism of Action & Administration \\
\hline Biguanide & Decrease insulin resistance & Oral \\
Sulfonylurea & Stimulate insulin secretion & Oral \\
Meglitinide & Stimulate insulin secretion & Oral \\
$\begin{array}{l}\alpha \text {-glucosidase } \\
\text { inhibitor }\end{array}$ & Oral \\
Thiazolidinedione & Decrease carbohydrate absorption insulin resistance & Oral \\
Incretin-based drug: & Stimulate insulin secretion \\
GLP-1 analogue & & Injectable \\
Incretin-based drug: & Stimulate insulin secretion & \\
DPP-4 inhibitor & & Oral \\
SGLT2 inhibitor & Increase urinary glucose excretion & Oral \\
Insulin therapy & Increase insulin levels & Injectable \\
\hline Abbreviations: SGLT2, sodium-glucose co-transporter 2; GLP-1, glucagon-like peptide-1; DPP-4, dipeptidyl peptidase-4
\end{tabular}

Overall, all antidiabetic drugs have been associated with certain adverse events, and each drug has its own history and controversies. There is not one ideal drug with a perfect safety profile. Patients with type 2 diabetes require constant monitoring to ensure adequate glycemic targets and to assess the incidence of related adverse events. The next section focuses on malignancies as an adverse event among patients with type 2 diabetes. 


\subsection{Association between type 2 diabetes and cancer incidence}

Type 2 diabetes is associated with both macrovascular and microvascular complications. ${ }^{34}$ These complications result from the failure of traditional insulin uptake tissues (muscle, fat and liver) to respond to adequate levels of insulin over time. ${ }^{42}$ Another complication of type 2 diabetes is an increased risk of some cancers, ${ }^{5-122425}$ mediated by nontraditional insulin target tissues (e.g. breast and colorectal). These non-traditional tissues are thought to be less resistant to insulin, and are thereby hyper-stimulated by antidiabetic drugs, which may lead to mitogenic growth. ${ }^{66}$ Finally, increased insulin receptor content is a characteristic of certain cancer tissues, which supports the hypothesis of the role of insulin receptors in the biology of tumours. ${ }^{67} 68$

Several observational studies have reported an association between type 2 diabetes and cancer incidence; all reporting an increased risk of several cancers, with the exception of prostate cancer where a decreased incidence has been reported..$^{5-14}$ Figure 2, adapted from a meta-analysis on the association between type 2 diabetes and cancer, presents a summary of all associations. ${ }^{69}$ Observational studies have purported that the increase in cancer incidence may be due to shared risk factors like obesity, ${ }^{71012}$ or by the mitogenic effects of insulin. ${ }^{510}$ The increased risk may also depend on diabetes severity and disease management. ${ }^{10}$ With respect to colorectal cancer incidence among patient with type 2 diabetes, whereby patients are at an increased risk of $30 \%$, physical activity and BMI are the most important shared risk factors. ${ }^{6}$ The next section of this thesis will focus on colorectal cancer. 
Figure 2. Forest plot: type $\mathbf{2}$ diabetes and cancer incidence

\section{Analysis}

Prostate cancer incidence Lung cancer incidence Gastric cancer incidence Total cancer incidence Thyroid cancer incidence Ovarian cancer incidence Breast cancer incidence Colorectal cancer incidence Multiple myeloma incidence Non-Hodgkin's lymphoma incidence Leukemia incidence Esophageal cancer incidence Bladder cancer incidence Kidney cancer incidence Gallbladder cancer incidence ECC incidence Pancreatic cancer incidence ICC incidence Endometrial cancer incidence HCC incidence

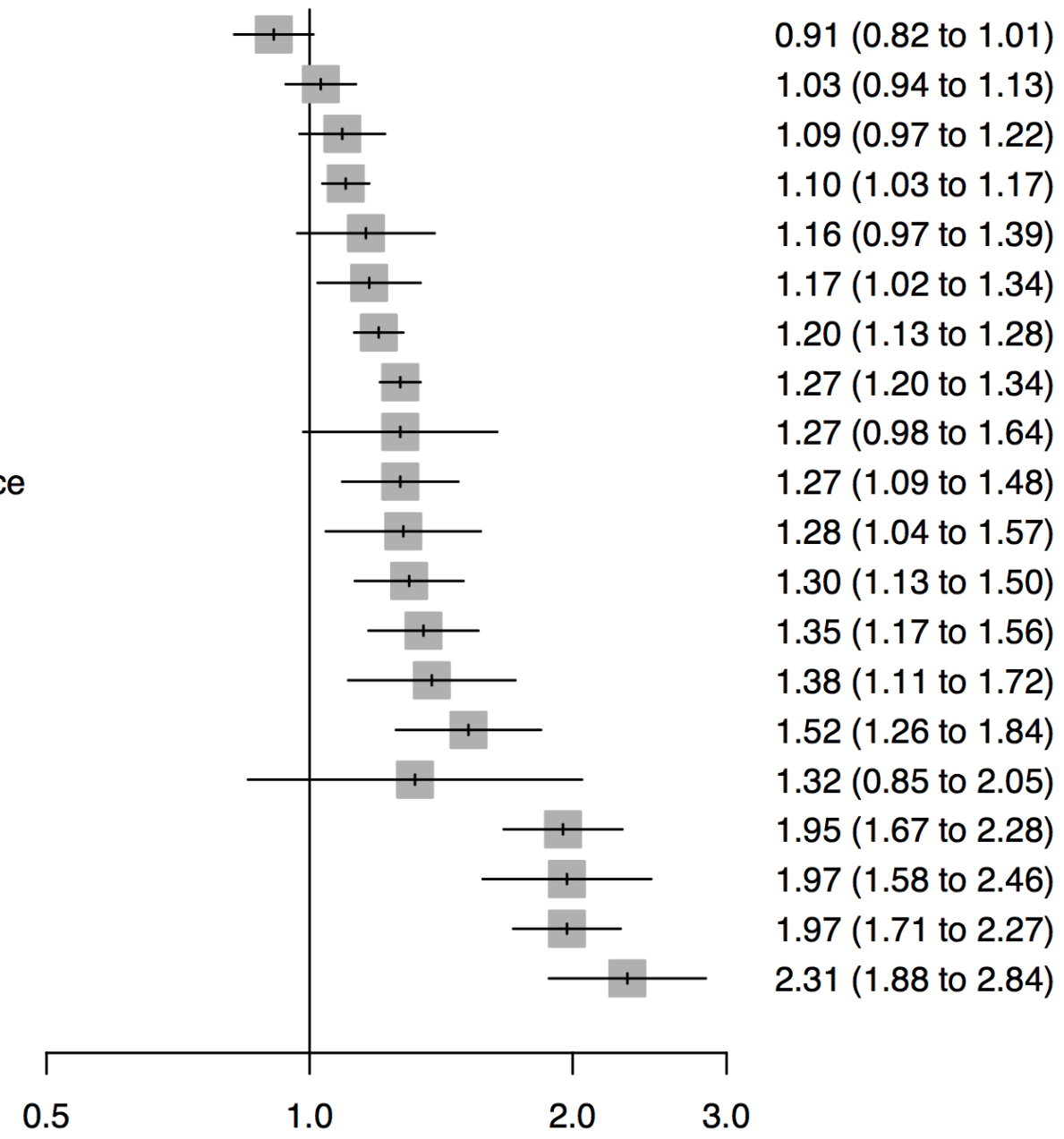

Abbreviations: ECC, extrahepatic cholangiocarcinoma; ICC, intrahepatic cholangiocarcinoma; HCC, hepatocellular carcinoma; HR, hazard ratio; Cl, confidence interval 


\subsection{Colorectal cancer}

\subsubsection{Epidemiology and risk factors}

Colorectal cancer is the second most commonly diagnosed cancer in Canada, responsible for $13 \%$ of new cancer diagnoses in $2016 .{ }^{70}$ Colorectal cancer is typically diagnosed through screening in the form of a colonoscopy or fecal occult blood testing. Through screening programs, there has been a temporal decline in colorectal cancer incidence, as screening allows for an increased detection of precancerous polyps. ${ }^{7172}$ About $50 \%$ of the reduction in incidence can be attributed to colorectal screening programs. ${ }^{73}$ In addition to an increase in screening, improvements to cancer care in high-income countries have contributed to decreasing trends in colorectal cancer mortality. ${ }^{74}$ However, colorectal cancer remains the second and third leading cause of death from cancer among Canadian men and women, respectively. ${ }^{70}$ As a prevalent disease, it is associated with a high economic burden; the management of this disease can cost an average of $\$ 20,000$ to $\$ 40,000$ per patient. ${ }^{75}$

Colorectal cancer typically develops from benign polyps lining the wall of the bowel. ${ }^{72}$ Through genetic mutations, loss of tumour suppression genes and activation of tumour promoting genes, these polyps increase in size and form a large cell mass, progressing to cancer over time. ${ }^{72}$ Coupled with these genetic factors are several modifiable and unmodifiable risk factors. Modifiable risk factors include physical inactivity, obesity, heavy alcohol and tobacco consumption, ${ }^{71}$ and a diet high in meat. ${ }^{72}$ Unmodifiable risk factors include age, whereby the likelihood of diagnosis increases after the age of 40 , as well as family history. ${ }^{71}$ Finally, about $3 \%$ of patients with colorectal cancer have a previous diagnosis of Lynch syndrome, the most common hereditary predisposition to colorectal cancer. ${ }^{76}$ 


\subsubsection{Association between type 2 diabetes and colorectal cancer}

As previously discussed, patients with type 2 diabetes are at an increased risk of colorectal cancer. ${ }^{6877}$ Due to many shared risk factors between the two aforementioned diseases, including physical inactivity and obesity, type 2 diabetes is often considered a risk factor for the development of colorectal cancer in of itself. ${ }^{6}$ This association is not the result of confounding, as studies controlling for physical inactivity and BMI as potential confounders report a significant positive association between colorectal cancer and type 2 diabetes (relative risk:1.34, 95\% confidence interval (CI): 1.20 to 1.49$).{ }^{6}$

A possible mechanism explaining the association between type 2 diabetes and colorectal cancer is that the hyperinsulinemia associated with early stage diabetes may promote colon carcinogenesis through the stimulation of insulin-like growth factor-I receptors. ${ }^{6}$ 877 Insulin is a growth factor for both colonic mucosal and cancer cells in vitro, and a colon tumour promoter in vivo. ${ }^{8}$ Thus, high levels of insulin may increase the risk of colorectal cancer. ${ }^{6877}$ As hyperinsulinemia is associated with early stage disease only, increased colorectal cancer incidence lessens with prolonged disease according to this mechanism. ${ }^{8}$ Other mechanisms explaining this association include a prolonged exposure to carcinogens in the bowel as a result of the slower bowel transit times among patients with type 2 diabetes. ${ }^{68}$ Patients with type 2 diabetes also have increased levels of fecal bile acids, resulting from their elevated glucose levels, which have been shown to be colorectal tumour promoting in animal studies. ${ }^{68}$

The association between type 2 diabetes and colorectal cancer may be modified by choice of antidiabetic drugs. Though the association with metformin has not been consistently 
observed, ${ }^{18} 78$ TZDs have been associated with a decreased risk of colorectal cancer. ${ }^{23}$ Evidence on the use of sulfonylureas is less conclusive. Their use has been associated with both an increased and decreased risk of overall cancer, ${ }^{2021}$ but there is no site specific association upon stratification. ${ }^{20}$ The next section of this thesis will focus on the incretin-based drugs and their potential association with colorectal cancer. 


\subsection{Colorectal cancer and the incretin-based drugs}

The incretin-based drugs, GLP-1 analogues and DPP-4 inhibitors, are a relatively new class of antidiabetic drugs, approved around a decade ago. ${ }^{79} 80$ As such, there is limited information on their long term safety. To date, associations between these drugs and some malignancies have been explored, ${ }^{30} 81$ however the information on colorectal cancer is limited. Assessing possible associations with long term adverse events is an important safety concern, as these drugs are becoming increasingly popular. This section will review what is currently known on the association between the incretin-based drugs and colorectal cancer.

\subsubsection{Biological evidence}

\subsubsection{Glucagon-like peptide-1 analogues}

The evidence on the safety of GLP-1 analogues with respect to colorectal cancer incidence is both limited and conflicting. On the one hand, there is a possible biological mechanism explaining how GLP-1 analogues may increase the proliferation of colorectal cancer. In this study, ApcMin/+ mice (colorectal mouse mutants) were treated with Exendin-4, a GLP-1 analogue. ${ }^{82}$ The treatment of Exendin-4 led to an increase in the number and size of polyps in the small bowel of these mice. ${ }^{82}$ Interestingly, this effect was not observed in Glp1r-l-:ApcMin/+ mice (GLP-1 receptor knockout mice), thus illustrating the importance of the

GLP-1 receptor. ${ }^{82}$ Finally, Exendin-4 also increased the expression of fibroblast growth factor 7 (FGF7) among ApcMin/+ mice, thus increasing intestinal growth. ${ }^{82}$ Therefore, GLP-1 analogues may enhance the growth of the small and large bowel via FGF7, increasing gut growth and crypt 
fission through the prolonged activation of the GLP-1 receptor. ${ }^{82}$ It is therefore possible that the interaction between GLP-1 analogues and FGF7 may promote gut hyperplasia and colon tumorigenesis.

However, there is conflicting evidence that treatment with Exendin-4 has anti-tumour properties in vitro, whereby Exendin-4 may alter cell morphology, initiate apoptosis and prevent proliferation of CT26 colon cancer cells. ${ }^{83}$ This effect is mediated by increased levels of cyclic AMP, suppression of glycogen synthase kinase 3 (GSK3) and extracellular signal-related kinase (ERK1/2), and reduction of cell viability. ${ }^{83}$ Inhibiting GSK3 and ERK1/2 reduces cell growth and promotes apoptosis; these kinases are targets for anti-cancer therapies. ${ }^{83}$ Exendin4 was also shown to have anti-tumour properties in vivo, whereby twice daily treatment induced apoptosis in CT26 tumorous BALB/c mice..$^{83}$ Finally, exenatide, another GLP-1 analogue, was also shown to have tumour-protecting effects in diabetic mice induced with colorectal cancer (1,2-dimethylhydrazine ). ${ }^{84}$ In this study, the cancerous mice had increased levels of angiogenic markers and cell proliferation compared with control mice, but these markers were downregulated upon treatment with Exenatide. ${ }^{84}$ Thus, exenatide's anti-angiogenic and antiproliferative mechanisms of action may contribute to observed chemo-preventative effects. ${ }^{84}$ Overall, the results of these two studies support anti-tumour effects of GLP-1 analogues.

\subsubsection{Dipeptidyl peptidase-4 inhibitors}

The biological evidence on the association between the use of DPP-4 inhibitors and colorectal cancer is limited, with in vitro and in vivo studies publishing conflicting results. There is some evidence that long term inhibition of the DPP-4 enzyme by DPP-4 inhibitors may lead to 
immune dysregulation and increased cancer risk. ${ }^{85}$ The DPP-4 enzyme regulates many T-cell functions, so its prolonged inhibition may compromise immunity and allow for the development and progression of cancer cells. ${ }^{85}$ There is also evidence that the use of DPP-4 inhibitors may support the metastasis of colon cancer cells. ${ }^{86}$ In vitro, the combined effects of DPP-4 inhibitors and GLP-2 (an incretin hormone) increased the proliferation and migratory activity of the HT29 colon cancer cell line ${ }^{86}$ The combination of GLP-2 and a DPP-4 inhibitor shortened the doubling time of HT29 cells compared with untreated control cells. ${ }^{86}$ In a repeated experiment using SW480 colon cancer cells, which display a lower proliferation rate than HT29, the addition of GLP-2 caused a decreased doubling time of SW480 cells compared with untreated cells. ${ }^{86}$ Adding a DPP-4 inhibitor did not further shorten the doubling time of these cells.$^{86} \mathrm{~A}$ possible hypothesis explaining this increased risk relates to the extended halflife of GLP-2 by the inhibition of the DPP-4 enzyme. GLP-2 has been associated with increased cell proliferation and migration; these tumour promoting effects may be enhanced by an increased half-life. ${ }^{86}$ As type 2 diabetes is a chronic disease, this effect is expected to exponentiate over time. ${ }^{86}$

In contrast, there is competing evidence showing a protective effect of these drugs. Treatment of the HT-29 cell line (colorectal) with DPP-4 inhibitors (sitagliptin and vildagliptin) showed decreasing cell viability at increasing drug concentrations, illustrating anticancer and cytotoxic activity in vitro. ${ }^{87}$ Similar effects were observed in vivo, whereby $1,2-$ dimethylhydrazine-induced rats (colonic cancer) treated with sitagliptin had significantly lower precancerous colorectal lesions and lower reactive oxygen species in the blood compared with control rats ${ }^{88} \mathrm{~A}$ possible explanation for this protective effect is the association between 
Vildagliptin and fibroblast activation protein (FAP), a protein overexpressed in cancer cells. ${ }^{87}$ In addition to its antidiabetic role, Vildagliptin has been shown to inhibit FAP and decrease the proliferation of lung cancer. ${ }^{89}$ As FAP is also overexpressed in colon cancer, this same mechanism may explain the observed protective role of DPP-4 inhibitors on colon cancer. ${ }^{87} 88$

\subsubsection{Safety reviews: United States Food and Drug Administration and European}

\section{Medicines Agency}

The US FDA and European Medicines Agency (EMA) independently review drug safety before market release based on data from RCTs. Their reviews of liraglutide, a GLP-1 analogue, highlighted some differences between treatment and control groups. Liraglutide exists in two formulations: $1.8 \mathrm{mg}$ as a diabetic dose and $3 \mathrm{mg}$ for weight management. Each dose will be discussed separately in this section.

\subsubsection{Liraglutide $1.8 \mathrm{mg}$}

In assessing the frequency of neoplasms among users of liraglutide $1.8 \mathrm{mg}$, the FDA compiled data from all completed phase 2 (intermediate-term) and phase 3 (long-term) blinded and open-label RCTs. ${ }^{34}$ In these trials, more subjects treated with liraglutide (78 subjects with 84 events [1.8\%]; 26.9 events per 1,000 person-years) than in the comparator group ( 29 subjects with 31 events [1.2\%]; 19.5 events per 1,000 person-years) reported total neoplasms. ${ }^{34}$ With respect to malignant colon cancer, there was no imbalance between the liraglutide group and the comparator group ( 2 subjects with 2 events [0.05\%]; 0.6 events per 1000 person-years and 1 subject with 1 event [0.04\%]; 0.6 events per 1000 person-years, respectively). ${ }^{34}$ However, 
more subjects in the liraglutide group reported malignant rectal cancer ( 2 subjects with 2 events [0.05\%]; 0.6 events per 1000 person-years) than in the control group (0 events reported). ${ }^{34}$

The EMA reported on clinical safety based on pooled data from 38 complete RCTs. ${ }^{90} \mathrm{~A}$ total of 32 malignant events were reported, with an observed imbalance between treatment and control groups (24 events, 8.9 per 1,000 person-years and 8 events, 5.3 events per 1,000 person-years, respectively). ${ }^{90}$ Aside from an imbalance of prostate and thyroid related neoplasms, all other site-specific rates were comparable between treatment and control groups. ${ }^{90}$ Similarly to the FDA, the EMA did not report an imbalance on the number of colon events. ${ }^{90}$ However, the EMA did not report an imbalance of rectal events ${ }^{90}$ as seen in the FDA report. $^{34}$

\subsubsection{Liraglutide $3 \mathrm{mg}$}

In assessing the safety of liraglutide $3 \mathrm{mg}$, the FDA reviewed data on all complete double-blind, randomized controlled weight loss trials, including one phase 2 and four phase 3 trials. A total of 3872 obese (BMI of $\geq 30 \mathrm{~kg} / \mathrm{m}^{2}$ ) or overweight (BMI of $\geq 27 \mathrm{~kg} / \mathrm{m}^{2}$ ) participants with at least one weight-related comorbidity (pre-diabetes, type 2 diabetes, hypertension, dyslipidemia, or obstructive sleep apnea) were studied. ${ }^{31}$ In these trials, more patients treated with liraglutide $3 \mathrm{mg}$ had colorectal cancer (2 events [0.06\%]; 0.4 events per 1000 person-years) compared with patients treated with placebo (no events reported). ${ }^{31}$ Finally, more patients treated with liraglutide had benign colorectal neoplasm compared with placebo (17 subjects 
with 17 events [0.52\%], 3.8 events per 1,000 person-years and 4 subjects with 4 events [0.22\%], 1.7 events per 1,000 person-years, respectively). ${ }^{31}$

The EMA reviewed the same 5 weight loss RCTs as the FDA. ${ }^{91}$ However, the EMA did not report an observed imbalance of colorectal events between treatment groups, only specifying that malignant colorectal neoplasms occurred at a very low rate. ${ }^{91}$ Moreover, they did not observe an imbalance in the number of benign colorectal neoplasms in the liraglutide group (11 subjects with 11 events [0.3\%]; 4 events per 1,000 person-years) versus placebo group (4 subjects with 4 events [0.2\%]; 3 events per 1,000 person-years). ${ }^{91}$

Overall, the reporting of the FDA and EMA was not entirely concordant; the FDA reported more details on imbalances with low number of events compared with the EMA. Due to the limited number of malignant colorectal events and short duration of follow-up (5 weeks - 56 weeks), ${ }^{31} 349091$ both agencies reported that continued monitoring of GLP-1 analogues with respect to neoplasms is required. Thus, the safety of the incretin-based drug class continued to be explored in post-marketing RCTs with longer follow-up time, discussed in the next section.

\subsubsection{Post-marketing randomized controlled trials}

Post-marketing RCTs gather additional information about drug efficacy after the drug has been approved by the FDA or EMA. There have been several post-marketing RCTs on the incretin-based drugs, mainly assessing their efficacy with respect to cardiovascular outcomes. This section will explore the primary findings of each RCT, as well as the findings on neoplasms. 


\subsubsection{Glucagon-like peptide-1 analogues}

This section will review the findings from four post-marketing RCTs on GLP-1 analogues that have been published to date (Table 2). The first trial is the Evaluation of Lixisenatide in Acute Coronary Syndrome (ELIXA) trial, which was designed to assess the safety of lixisenatide on cardiovascular outcomes. Participants with type 2 diabetes with a recent acute coronary syndrome were randomized 1:1 in a double-blind, placebo-controlled trial. ${ }^{92}$ The RCT included 6098 patients from 49 countries; 3034 randomized to lixisenatide versus 3034 randomized to placebo..$^{22}$ Over a median follow-up of 2.1 years, the primary endpoint (death from cardiovascular causes, nonfatal myocardial infarction, nonfatal stroke, or hospitalization for unstable angina) occurred in equal rates between treatment and control groups (406 events [13.4\%] and 399 events [13.2\%], respectively). With respect to neoplasms, there was no imbalance in the number of events in the treatment group compared with the control group (72 events [2.4\%] and 61 events [2.0\%], respectively)..$^{92}$ In terms of site specific cancers, there was no record of colorectal neoplasms; thus no imbalance of colorectal events was observed between treatment groups in the ELIXA trial. ${ }^{92}$

The Trial to Evaluate Cardiovascular and Other Long-term Outcomes with Semaglutide in Subjects with Type 2 Diabetes (SUSTAIN-6) was a non-inferiority trial designed to assess cardiovascular safety among patients with type 2 diabetes. This randomized, double-blind, parallel-group trial involved assessing the safety of $0.5 \mathrm{mg}$ once-weekly semaglutide versus placebo, and $1.0 \mathrm{mg}$ once-weekly semaglutide versus placebo. ${ }^{93}$ This RCT randomized 3297 participants 1:1:1:1 to the $0.5 \mathrm{mg}(\mathrm{n}=826)$ dose of semaglutide or volume matched placebo $(n=824)$, and to the $1.0 \mathrm{mg}(\mathrm{n}=822)$ dose of semaglutide or volume matched placebo $(\mathrm{n}=825) .{ }^{93}$ 
The primary endpoint was death from cardiovascular causes or nonfatal myocardial infarction or stroke, which occurred in 108 participants in the semaglutide group [6.6\%] and 146 participants in the placebo group [8.9\%]. ${ }^{93}$ The observed hazard ratio (HR: $0.74,95 \% \mathrm{Cl}: 0.58$ to 0.95) confirmed the non-inferiority of semaglutide compared with placebo, with no difference between the two doses. ${ }^{93}$ In assessing other long-term outcomes, the rates of malignant neoplasms were similar across treatment and control groups (66 events [4.0\%] and 70 events [4.2\%], respectively), but were higher among the $1.0 \mathrm{mg}$ dose of semaglutide (40 events [4.9\%]) then the $0.5 \mathrm{mg}$ dose (26 events [3.1\%]). ${ }^{93}$ Though there was a numerical imbalance in the number of events of colorectal cancer among treatment and control groups (4 events [0.2\%] and 8 events [0.5\%], respectively), this difference was not significant. ${ }^{93}$

The Liraglutide Effect and Action in Diabetes: Evaluation of Cardiovascular Outcome Results (LEADER) trial is the longest post-marketing trial to date, accruing a median of 3.8 years of follow-up. ${ }^{94}$ This double-blind, placebo-controlled trial was designed to assess the cardiovascular safety of the $1.8 \mathrm{mg}$ dose of liraglutide among participants with type 2 diabetes at high risk for cardiovascular disease. ${ }^{94}$ The primary outcome (death from cardiovascular disease, nonfatal myocardial infarction or nonfatal stroke) was assessed among 9340 patients randomized 1:1 to liraglutide $(n=4668)$ or placebo $(n=4672) \cdot{ }^{94}$ Indeed, the primary outcome occurred at a significantly lower rate (HR: $0.87,95 \% \mathrm{Cl}: 0.78$ to 0.97$)$ among the treatment group, with a lower event rate among those randomized to liraglutide (608 events, [13.0\%]) than in the placebo group (694 events [14.9\%]). ${ }^{94}$ This trial also assessed other adverse events, and found similar rates of malignant neoplasms in treatment (296 events [6.3\%]) and control groups (279 events [6.0\%]).${ }^{94}$ When looking at site specific cancers, there was no imbalance in 
the number of colorectal events among treatment and control groups ( 28 events [0.6\%] in each group)..$^{94}$

The last post-marketing trial of interest was designed to assess the safety of liraglutide 3 mg for weight loss among non-diabetic overweight or obese participants. The SCALE trial was designed as a placebo-controlled trial, whereby 3731 participants were randomized 2:1 to liraglutide injection or placebo. ${ }^{95}$ After 56 weeks, body weight was measured to determine weight change from baseline, and all other adverse events were recorded. ${ }^{95}$ Patients randomized to liraglutide had a significant reduction in weight loss compared to placebo; the liraglutide users had lost a mean ( \pm standard deviation (SD)) of $8.0 \pm 6.7 \%$ of their body weight while the patients randomized to placebo who had lost a mean of $2.6 \pm 5.7 \%$ of their body weight. ${ }^{95}$ The incidence of neoplasms was similar in treatment (1.9 events per 100 personyears) and placebo groups (2.4 events per 100 person-years), and there was no specification with regards to colorectal cancer. ${ }^{95}$ 
Table 2. Post-marketing randomized controlled trials: glucagon-like peptide-1 analogues

\begin{tabular}{|c|c|c|c|c|}
\hline Trial & Drug & $\begin{array}{c}\text { Number of } \\
\text { patients }\end{array}$ & $\begin{array}{l}\text { Median follow- } \\
\text { up time (years) }\end{array}$ & $\begin{array}{l}\text { Colorectal } \\
\text { events (n) }\end{array}$ \\
\hline \multirow[t]{2}{*}{ ELIXA } & Lixisenatide & 3034 lixisenatide & 2.1 & None reported \\
\hline & & 3034 placebo & & \\
\hline \multirow[t]{3}{*}{ SUSTAIN-6 } & Semaglutide & 1648 & 2.1 & Treatment: 4 \\
\hline & & semaglutide & & Placebo: 8 \\
\hline & & 1649 placebo & & \\
\hline \multirow[t]{2}{*}{ LEADER } & Liraglutide $^{a}$ & 4668 liraglutide & 3.8 & Treatment: 28 \\
\hline & & 4672 placebo & & Placebo: 28 \\
\hline \multirow[t]{2}{*}{ SCALE } & Liraglutide $^{b}$ & 2487 liraglutide & 1.1 & None reported \\
\hline & & 1244 placebo & & \\
\hline
\end{tabular}

\footnotetext{
${ }^{a}$ diabetic dose $(1.8 \mathrm{mg})$

${ }^{\mathrm{b}}$ weight management $(3 \mathrm{mg})$
}

Reviewing the post-marketing trials on GLP-1 analogues showed conflicting findings.

Though there was a numerical imbalance in the number of colorectal events in the SUSTAIN-6 trial, this imbalance was not statistically significant. ${ }^{93}$ Furthermore, the remaining postmarketing trials on GLP-1 analogues did not report an imbalance of colorectal events. ${ }^{92} 9495$ RCTs are neither designed nor powered to assess cancer incidence, so further studies are required to assess the true nature of this association.

\subsubsection{Dipeptidyl peptidase-4 inhibitors}

There are three post-marketing RCTs assessing the safety of DPP-4 inhibitors (Table 2). The first is the Examination of Cardiovascular Outcomes with Alogliptin versus Standard of Care 
(EXAMINE) trial, which was designed to assess risk of adverse cardiovascular events among users of Alogliptin. In this randomized double-blind trial, 5380 patients with type 2 diabetes were randomized 1:1 to alogliptin or placebo, and followed for a median of 1.5 years. ${ }^{96}$ The primary endpoint (death from cardiovascular cause or nonfatal myocardial infarction or stroke), occurred in similar rates between the treatment and control groups ( $11.3 \%$ and $11.8 \%$, respectively).${ }^{96}$ Furthermore, users of alogliptin had similar rates of other serious adverse events (33.6\%) (including cancer) compared with placebo (35.5\%), whereby no site specific rates were reported. ${ }^{96}$ Therefore, there was no imbalance in the number of colorectal events among users of alogliptin and placebo in the EXAMINE trial. ${ }^{96}$

The Trial Evaluating Cardiovascular Outcomes with Sitagliptin (TECOS) was designed to assess the safety of sitagliptin with respect to cardiovascular outcomes. In this trial, 14,671 patients with type 2 diabetes and established cardiovascular disease were randomized 1:1 to sitagliptin $(n=7332)$ or placebo $(n=7339) .{ }^{97}$ Over a median follow-up of 3.0 years, participants were followed to assess the primary adverse event (cardiovascular death, nonfatal myocardial infarction or stroke, or hospitalization for unstable angina) as well as a number of other adverse events, including cancer. ${ }^{97}$ Overall, there was no difference in rates of the primary outcome between treatment (839 events [11.4\%], 4.06 per 100 person-years) and control groups (851 events [11.6\%], 4.17 per 100 person-years). ${ }^{97}$ There was also no significant difference between the two groups in terms of cancer incidence (268 events [3.7\%], 1.25 per 100 person-years versus 290 events [4.0\%], 1.37 per 100 person-years, respectively), with no instances of colorectal cancer reported. ${ }^{97}$ 
The Saxagliptin Assessment of Vascular Outcomes Recorded in Patients with Diabetes Mellitus (SAVOR)-Thrombolysis in Myocardial Infarction (TIMI) 53 trial of participants with type 2 diabetes was designed to assess the cardiovascular safety of saxagliptin. After this study was published, a post-hoc analysis was conducted to explore the possible association between saxagliptin use and cancer; made possible because of the length of follow-up (median 2.1 years)..$^{32}$ Overall, 688 participants (4.1\%) reported at least one cancer event, with no difference in rates between treatment and placebo groups (362 events [4.3\%] and 326 events [3.8\%], respectively). ${ }^{32}$ However, there was a statistically significant difference between treatment and control groups in terms of colon cancer (16 events versus 31 events respectively, HR: 0.51, 95\% $\mathrm{Cl}: 0.27=0.92) .{ }^{32}$ This study was the first to report a possible protective effect of saxagliptin, whereby its use was associated with a $49 \%$ decreased risk of colon cancer. ${ }^{32}$

In contrast, the results of a pooled analysis of sitagliptin trials did not show a decreased risk of colon cancer, ${ }^{33}$ as observed in the SAVOR-TIMI 53 RCT analysis. ${ }^{32}$ In this analysis, data from a total of 14,611 participants from 25 RCTs were examined to determine if rates of adverse events were higher among treatment or control groups. ${ }^{33}$ This section will focus on rates of neoplasms, and site specific rates of colon and rectal cancers. Overall, the incidence rate of any malignant, benign or unspecified neoplasms was slightly higher among the treatment group compared with the control group (incidence rate 2.0 per 100 person years and 1.5 per 100 person years, respectively). ${ }^{33}$ Site specifically, the incidence rate of colon cancer was higher among the treatment group (0.09 per 100 person-years), compared with the placebo group (0.04 per 100-person years), but there was no difference in the incidence rate of rectal cancer (0.02 per 100 person-years in both groups) between the two groups. ${ }^{33}$ 
Table 3. Post-marketing randomized controlled trials: dipeptidyl peptidase-4 inhibitors

\begin{tabular}{|c|c|c|c|c|}
\hline Trial & Drug & $\begin{array}{c}\text { Number of } \\
\text { patients }\end{array}$ & $\begin{array}{l}\text { Median follow- } \\
\text { up time (years) }\end{array}$ & $\begin{array}{l}\text { Colorectal } \\
\text { events }(n)\end{array}$ \\
\hline EXAMINE & Alogliptin & $\begin{array}{l}2701 \text { alogliptin } \\
2679 \text { placebo }\end{array}$ & 1.5 years & None reported \\
\hline TECOS & Sitagliptin & $\begin{array}{l}7332 \text { sitagliptin } \\
7339 \text { placebo }\end{array}$ & 3.0 years & None reported \\
\hline SAVOR-TIMI $53^{\text {a }}$ & Saxagliptin & $\begin{array}{c}8280 \text { saxagliptin } \\
8212 \text { placebo }\end{array}$ & 2.1 & $\begin{array}{c}\text { Treatment: } 16 \\
\text { Placebo: } 31\end{array}$ \\
\hline
\end{tabular}

a imbalance of colon events observed in post-hoc analysis

Overall, data from post-marketing RCTs on the safety of DPP-4 inhibitors and their posthoc analyses have been contradictory. Though an analysis of the SAVOR-TIMI 53 RCT showed a significant protective effect associated with use of saxagliptin, ${ }^{32}$ the pooled analysis described above showed an increase in incidence among users of sitalgiptin. ${ }^{33}$ The remaining trials did not present any imbalances. ${ }^{96} 97$ These discordant results highlight the need for observational studies, properly designed to asses cancer incidence, to study this important association.

\subsubsection{Observational studies}

To date, there has been one observational study assessing the association between the use of the incretin-based drugs and colorectal cancer. ${ }^{35}$ Using US Medicare data, the authors identified three cohorts of second-line drugs: DPP-4 inhibitors versus TZDs, DPP-4 inhibitors versus sulfonylureas and GLP-1 analogues versus long-acting insulin. ${ }^{35}$ To be included in a cohort, patients must not have been treated with either of the antidiabetic drugs under 
comparison in the 12 months prior to initial prescription (6 months for the GLP-1 analogue analysis due to small sample size). ${ }^{35}$ Finally, all patients were required to have a second prescription within 30 days (90 for injections) to increase the probability that patients actually took the drugs. ${ }^{35}$ In an as treated definition, exposure was defined from 6 months after the time of the second prescription until 6 months after stopping, switching or augmenting the drug. ${ }^{35}$ Within this period, an incidence of colorectal cancer would be defined as exposed. ${ }^{35}$ To control for confounding, propensity score weights were used. ${ }^{35}$

Overall, this study reported no association between the use of the incretin-based drugs and incident colorectal cancer. ${ }^{35}$ The median duration of treatment among the DPP-4 inhibitor cohorts was 0.7 to 0.9 years. ${ }^{35}$ Compared with TZDs, there was no association between the use of DPP-4 inhibitors and colorectal cancer (adjusted HR: $1.19,95 \% \mathrm{Cl}: 0.88$ to 1.71 ). ${ }^{35}$ There was also no association compared with sulfonylureas (adjusted HR: $0.98,95 \% \mathrm{Cl}: 0.74$ to 1.30 ). ${ }^{35}$ Among a median duration of use of 0.8 to 1.2 years in the GLP-1 analogue cohort, their use was not associated with the incidence of colorectal cancer compared with the use of long-acting insulin (adjusted HR: $0.82,95 \% \mathrm{Cl}: 0.42$ to 1.58$).{ }^{35}$

This study has some limitations, and as such its findings must be interpreted with caution. Most importantly, it had a short duration of follow-up ( 0.7 to 1.2 years)..$^{35}$ This length of time may be too short to assess cancer incidence. Additionally, the database used in this study does not include information on smoking, alcohol use and BMI, and thus the authors were not able to adjust for these potential confounders. ${ }^{35}$ Finally, the as-treated definition used in the primary analysis is inappropriate to assess cancer incidence. An as treated analysis assumes the effect of the drug immediately reverses upon discontinuation, whereas an 
intention to treat analysis assumes irreversibility. For cancer outcomes, the latter appears to be the more appropriate assumption.

To conclude, the available evidence on the association between the use of the incretinbased drugs and incident colorectal cancer is contradictory. As the incretin-based drugs are a popular antidiabetic drugs, assessing this association is an important safety concern. The remainder of this thesis will outline how I studied this association using a large populationbased cohort study. 


\section{Chapter 3: Objectives and hypotheses}

\subsection{Objective}

The main objective of this thesis is to determine whether the use of the incretin-based drugs GLP-1 analogues and DPP-4 inhibitors, is associated with incident colorectal cancer in patients with type 2 diabetes.

\subsubsection{Secondary objectives}

This thesis has four secondary objectives:

1) To determine whether there is a duration-response relation between cumulative duration of drug use ( $\leq 1$ year, 1.1-2 years and $>2$ years) and the incidence of colorectal cancer.

2) To determine if there is an association between time since drug initiation ( $\leq 2$ years and $>2$ years) and incident colorectal cancer.

3) To determine if there is an association between the most common individual drug types within each incretin-based drug class (GLP-1 analogues: exenatide and liraglutide; DPP-4 inhibitors: sitagliptin, saxagliptin) and colorectal cancer.

4) To determine if there is an association between the use of incretin-based drugs and colon and rectal cancer, separately. 


\subsection{Hypothesis}

The primary hypothesis is that there is no association between the use of the incretinbased drugs and incident colorectal cancer.

\subsubsection{Secondary hypotheses}

1) There is no duration response relationship between use of incretin-based drugs and the incidence of colorectal cancer.

2) There is no association between the time since initiation of incretin-based drugs and incident colorectal cancer.

3) There is no association by commonly prescribed individual drug types.

4) There is no association when stratifying on colon versus rectal cancer. 


\section{Chapter 4: Methodology}

The methodology for this project is described in detail in the manuscript (Chapter 5). In this section, some items of the methodology are described in a more detail. These include additional information on the data source, a description and explanation for the use of the base-study-cohort approach, and a more detailed presentation of the definition of the exposure and outcome. Finally, a description of the disease risk score method, marginal structural modelling and multiple imputation will be presented, which are used in sensitivity analyses.

\subsection{Data source}

We used the UK Clinical Practice Research Datalink (CPRD) as the data source for this study. The CPRD was established in 1987 as the Value Added Medical Products dataset. ${ }^{98}$ In 1993 it expanded to the General Practice Research Database, before becoming the CPRD in $2012 .{ }^{98}$ The CPRD is a continuously growing primary care dataset, whereby patient records from general practices are updated monthly. ${ }^{98}$ The CPRD is linkable to a number of secondary care datasets, including hospitalization data from Hospital Episode Statistics repository, mortality data from the Office for National Statistics, and cancer registration data from the National Cancer Intelligence Network. ${ }^{98}$

The CPRD is considered one of the largest longitudinal databases around the world, encompassing data from over 15 million patients from 700 general practices around the UK. ${ }^{98}$ Recorded data in the CPRD includes information on demographics, diagnoses, prescriptions and referrals. The representativeness of the CPRD has been studied by the comparing the CPRD 
population to other validated sources. Compared to the 2011 census, CPRD patients are similar to the general population in terms of age, sex and ethnicity, and are comparable for BMI distribution when compared to the Health Survey for England. ${ }^{98}$ Further, data have been validated in a number of different studies, whereby high positive predictive values and high sensitivity are indicative of valid and complete data; ${ }^{98-100}$ diabetes recording has a sensitivity in excess of $90 \% .{ }^{99}$ Finally, cancer diagnoses are highly concordant $(83.3 \%)$ with the UK National Cancer Data Repository, and completeness of case ascertainment has been as high as $98 \%$ for colorectal cancer. ${ }^{101}$

Due to the validity and quality of data, the CPRD has been used as a data source in a number of observational studies to study the safety of antidiabetic drugs on a number of different outcomes. ${ }^{20242930}$ The CPRD has also been used to assess the risk of colorectal cancer associated with the use of different drugs. ${ }^{102} 103$ Finally, the CPRD has been used to assess the safety of antidiabetic drugs on incident colorectal cancer. ${ }^{78} 104105$ Thus, the CPRD was deemed to be an appropriate data source to address our important safety question.

\subsection{Base-study-cohort approach}

The study population was defined using a base-study-cohort approach, described in detail in Chapter 5. In short, we identified two cohorts; the base cohort represented those newly-treated with antidiabetic drugs from 1988 and onwards, and the study cohort was comprised of new users of antidiabetic drug in or after 2007 (the year the first incretin-based drug entered the UK market). The date of the new antidiabetic prescription is the date of cohort entry. We used data from the study cohort for all analyses. 
The base-study cohort approach is a favourable design for a number of reasons. Without the base cohort, we would be restricted to data from 2007 and onward, which would severely limit the size of our cohort and the type of patients included. As the incretin-based drugs are not indicated as a first-line treatment, users typically represent those with long-term disease. Thus, using the base-study approach allows for the identification of new users of incretin-based drugs based on their previous drug history (included in the base cohort). The base cohort also allows for a more accurate calculation of the duration of treated diabetes, an important proxy for disease severity. Lastly, all newly-treated patients represent users who are not well managed, likely to have similar disease severities thereby minimizing potential confounding by indication.

This design is shown schematically in Figure 3. Patients $A$ and $B$ represent those who initiated a new class of antidiabetic drug in or after 2007 respectively, thus are part of the study cohort only. Patient $\mathrm{C}$ is part of the base cohort from the time of their first prescription, until their switch or add-on in 2007, from which point forward they are in the study cohort. Patient D represents someone who initiated a new antidiabetic drug, followed by a switch or add-on before the incretin-based drugs entered the market, and had a second switch after 2007. This patient only becomes part of the base cohort at the time of their second switch or add-on. Finally, patients $\mathrm{E}$ and $\mathrm{F}$ do not initiate a new class of antidiabetic drug in or after 2007, and thus do not go into the study cohort.

The base-cohort study design has been successfully used in a number of observational studies assessing the safety of antidiabetic drugs on cancer incidence. Some examples include the use of glyburide and overall cancer risk, ${ }^{20}$ the use of the incretin-based drugs and pancreatic 
cancer, ${ }^{29}$ the use of the incretin-based drugs and breast cancer ${ }^{30}$ and the use of pioglitazone and bladder cancer..$^{24}$ Thus, this study design was appropriate to assess the association between the use of the incretin-based drugs and colorectal cancer. 
Figure 3. Base-study cohort schematic
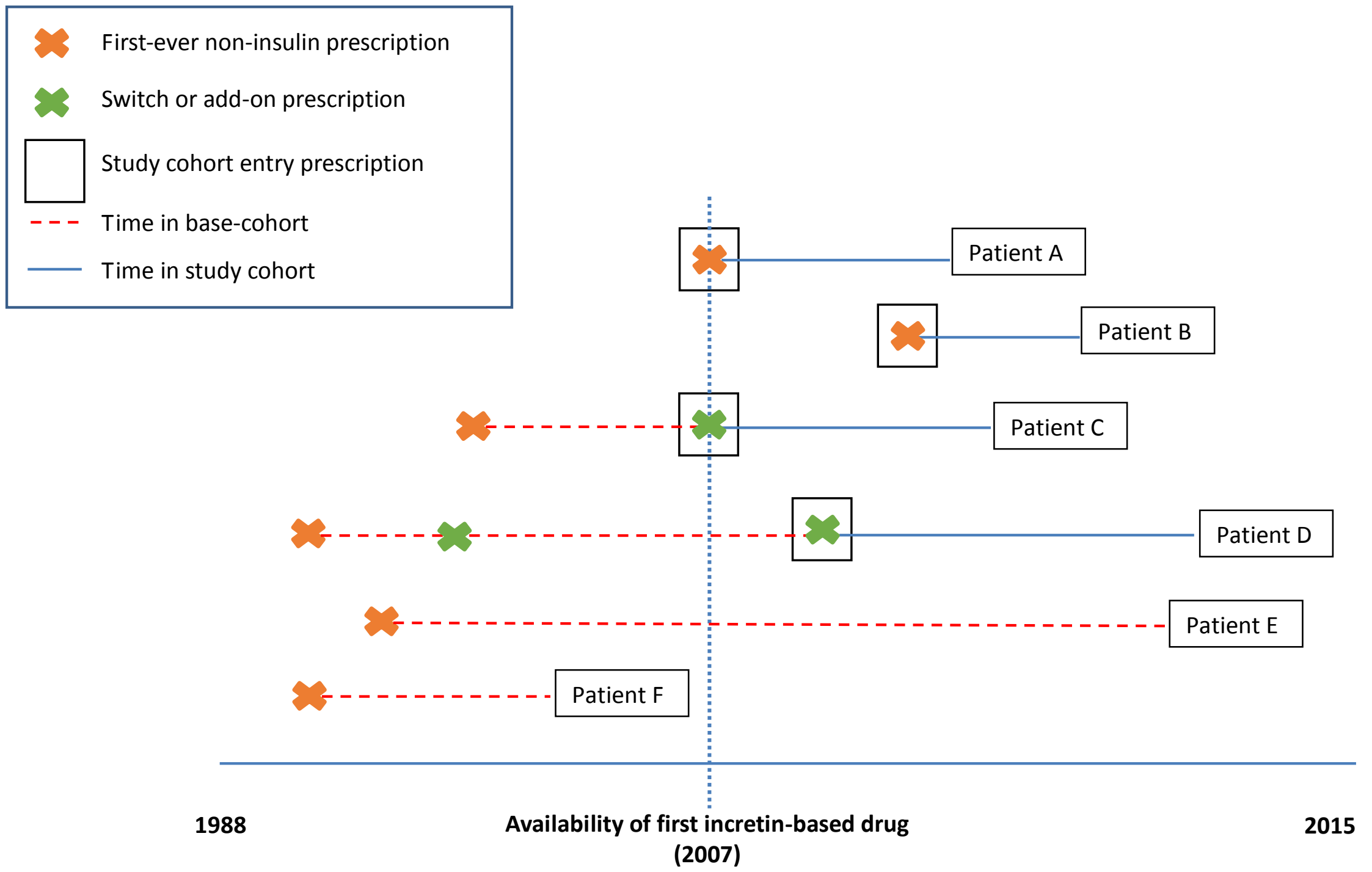


\subsection{Exposure definition}

Exposure to GLP-1 analogues and DPP-4 inhibitors was defined in a hierarchical, timedependent fashion, presented in detail in Chapter 5. As described above, cohort entry is the date of a new antidiabetic prescription, whereby patients are considered exposed to the drug after a 1-year lag period. This lag period is necessary for latency, and to minimize reverse causality and detection bias. As the outcome was the incidence of colorectal cancer, we are interested in the ever use of the drug, analogous to an intention to treat approach. The reference category for all analyses was the use of sulfonylureas, a clinically relevant second-tothird-line antidiabetic drug. This definition will be presented in Figures $\mathbf{4}$ and $\mathbf{5}$ for exposure to GLP-1 analogues only (the same definition is used for the separate analysis of DPP-4 inhibitors).

In Figure 4, participants are exposed to a single drug only. Patient $A$ is exposed to the drug of interest, GLP-1 analogues, but contributes person time to the unexposed category until 1-year after the initial prescription. From that point forward, this person contributes persontime to the exposed group, thus an event occurring in this period would be an exposed event. Patient B is exposed to sulfonylureas only, the reference category. After the 1-year lag period, the patient continues to be unexposed to the drug of interest, thus an event occurring in this time would be classified as unexposed. 


\section{Figure 4. Exposure definition: single drug exposure}

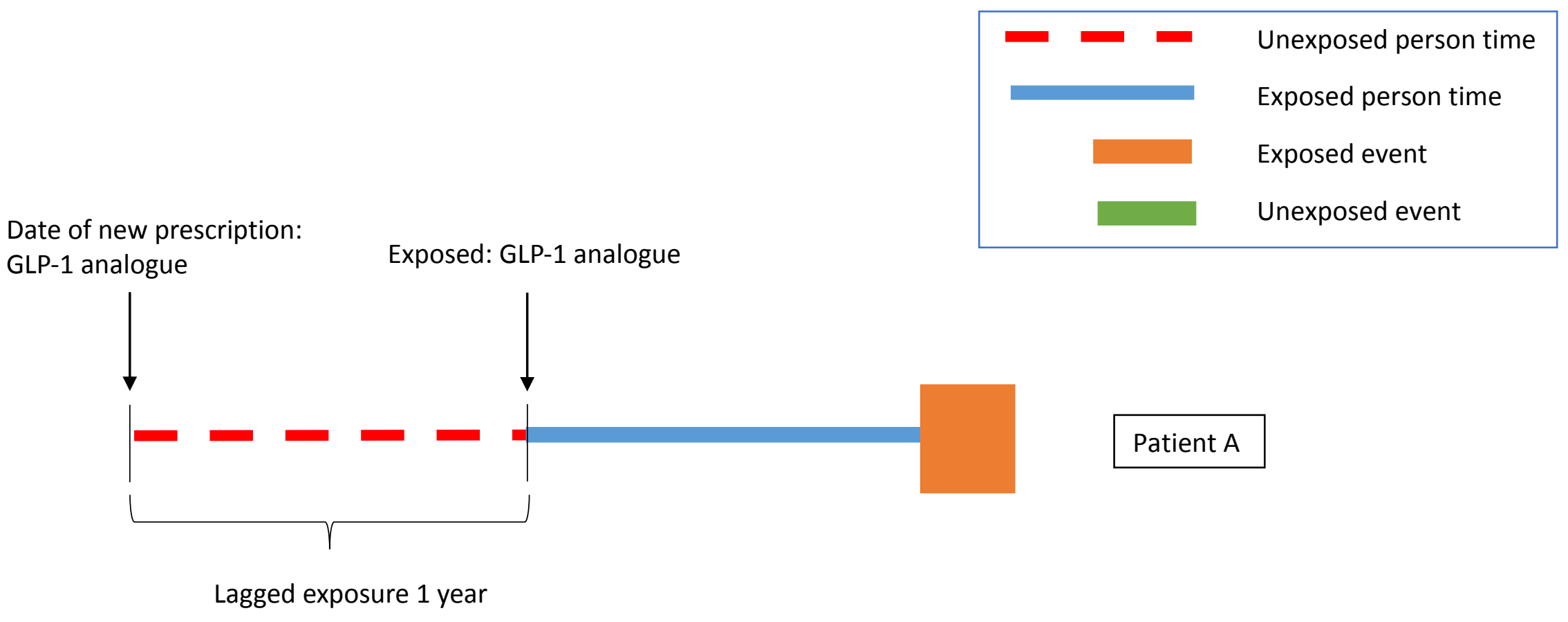

Date of new prescription:

Sulfonylurea

Exposed: Sulfonylurea

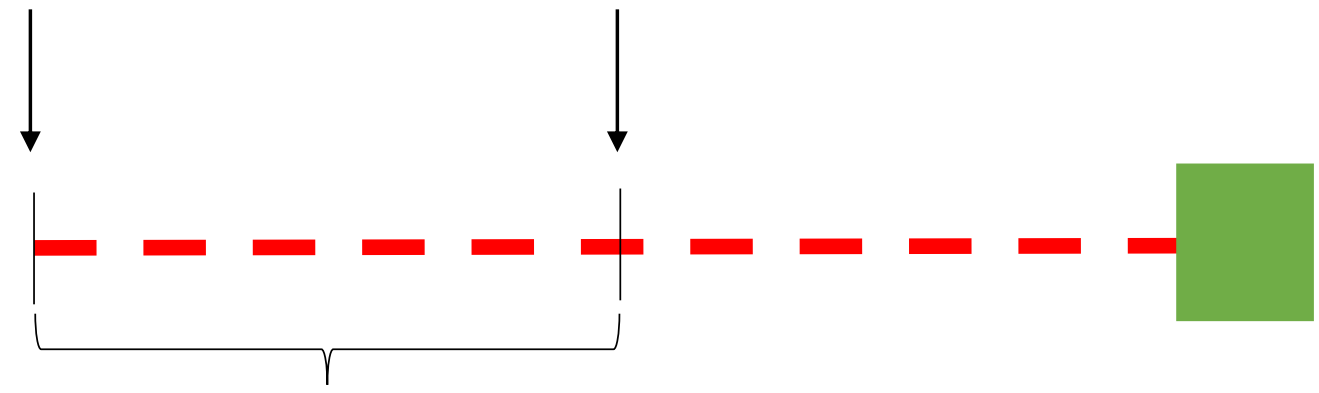

Patient B

Lagged exposure 1 year 
Figure 5 represents some more complex exposure patterns, whereby patients have a switch or add-on in their treatment history. Patient $A$ is considered unexposed to all drugs until 1-year after their initial exposure; at this time they become exposed to the reference category, sulfonylureas. After some time, this patient is prescribed the drug of interest, GLP-1 analogues, as an add-on to treatment or a switch. This patient has a colorectal event within the first year of exposure to GLP-1 analogues, and as such this event is classified as unexposed. This example illustrates the importance of the lag period, which occurs after each new prescription. Patient B highlights the hierarchical definition, whereby exposure is defined as the use of GLP-1 analogues, then use of sulfonylureas and then use of all other antidiabetic drugs. As usual, the patient is considered exposed to the drug of interest after the 1-year lag period. After some time, the patient has a new prescription for sulfonylureas. Even after the lag period, this patient is considered exposed to the primary drug of interest (and not to sulfonylureas), as use of GLP-1 analogue is the first level of the hierarchy. Thus, once exposed to GLP-1 analogues, patients are considered exposed for the remainder of the study period (intention to treat). Therefore patient B contributes an exposed event. 
Figure 5. Exposure definition: multiple drug exposures

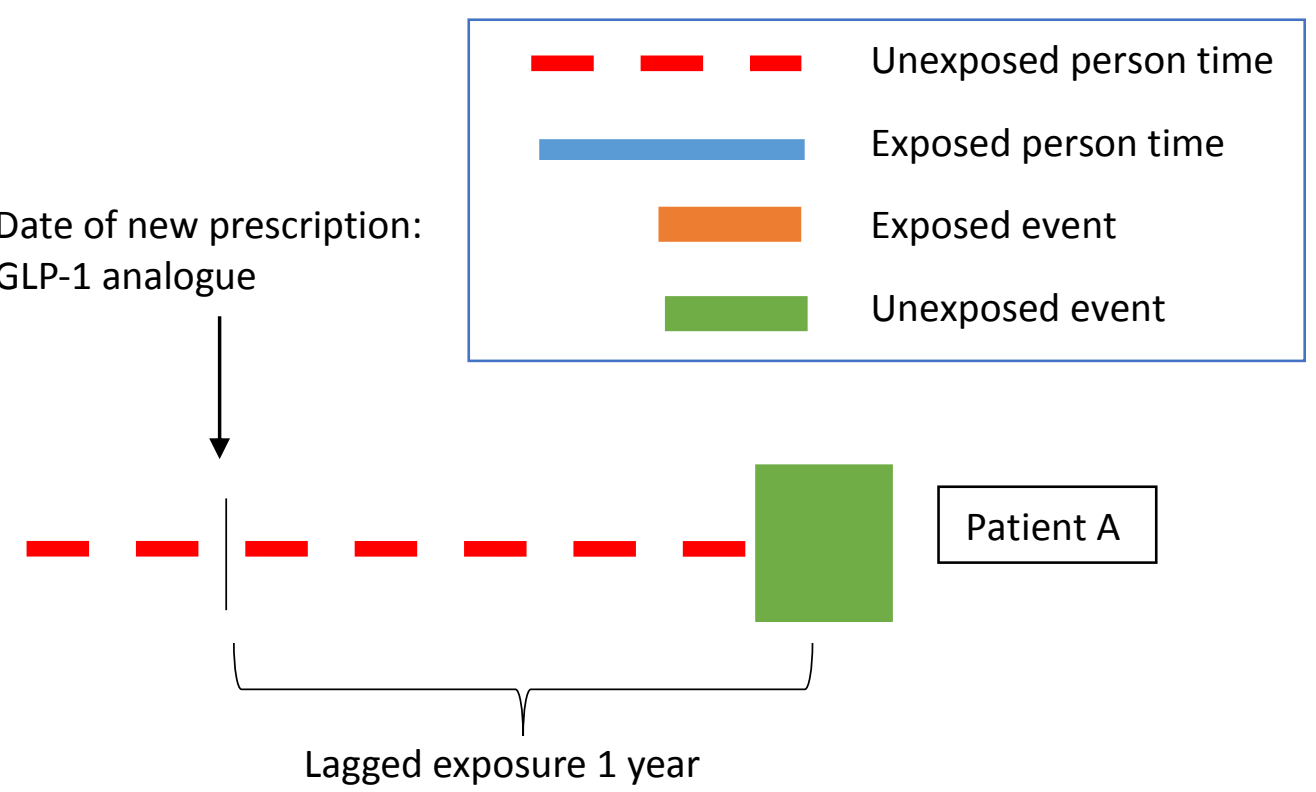

Date of new prescription:

GLP-1 agonist

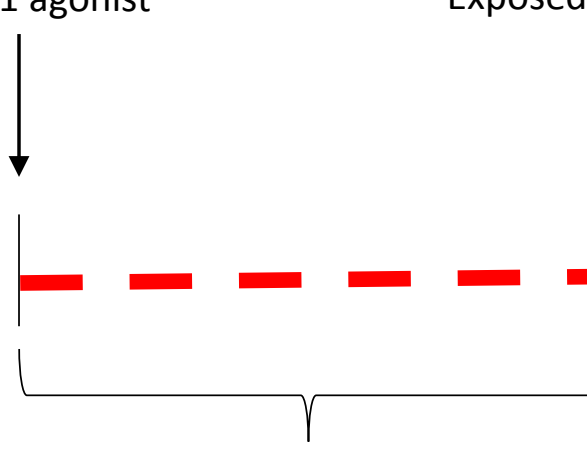

Lagged exposure 1 year
Exposed: sulfonylurea

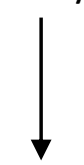

Date of new prescription:

sulfonylurea

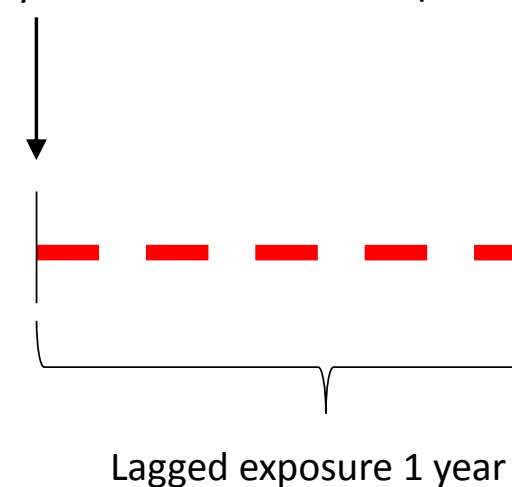

Lagged exposure 1 year

Date of new prescription:

sulfonylurea

Patient B 


\subsection{Outcome definition}

Patients in the study cohort were followed until a first-ever diagnosis of colorectal cancer, or censored upon death from any cause, end of registration with the general practice or end of the study period, whichever came first. We identified cases of colorectal cancer according to read codes, which are used in the CPRD to record medical diagnoses and procedures (Table 4). Read codes for colorectal cancer have been previously validated compared to the UK cancer registry, which is considered the gold standard for cancer recordings. ${ }^{106}$ Compared to the cancer registry, the CPRD is associated with a high sensitivity and specificity (92\% and 99\%, respectively) and with a high positive predictive value (98\%); the CPRD captured 1732 of 1882 colorectal cancer diagnoses. ${ }^{106}$ Thus, the read code classification of colorectal cancer diagnoses used in the CPRD is highly valid. 
Table 4. Read codes: colorectal carcinoma

\begin{tabular}{|c|c|}
\hline Readcode & Readterm \\
\hline B13..00 & Malignant neoplasm of colon \\
\hline B141.00 & Malignant neoplasm of rectum \\
\hline B133.00 & Malignant neoplasm of sigmoid colon \\
\hline B134.00 & Malignant neoplasm of caecum \\
\hline B141.12 & Rectal carcinoma \\
\hline B131.00 & Malignant neoplasm of transverse colon \\
\hline B141.11 & Carcinoma of rectum \\
\hline B130.00 & Malignant neoplasm of hepatic flexure of colon \\
\hline B13z.11 & Colonic cancer \\
\hline B132.00 & Malignant neoplasm of descending colon \\
\hline B136.00 & Malignant neoplasm of ascending colon \\
\hline B902500 & Neoplasm of uncertain behaviour of rectum \\
\hline B137.00 & Malignant neoplasm of splenic flexure of colon \\
\hline B902400 & Neoplasm of uncertain behaviour of colon \\
\hline B134.11 & Carcinoma of caecum \\
\hline B140.00 & Malignant neoplasm of rectosigmoid junction \\
\hline B13z.00 & Malignant neoplasm of colon NOS \\
\hline B14..00 & Malignant neoplasm of rectum, rectosigmoid junction and anus \\
\hline B13y.00 & Malignant neoplasm of other specified sites of colon \\
\hline B14z.00 & Malignant neoplasm rectum, rectosigmoid junction and anus NOS \\
\hline B14y.00 & Malig neop other site rectum, rectosigmoid junction and anus \\
\hline B138.00 & Malignant neoplasm, overlapping lesion of colon \\
\hline B1z0.11 & Cancer of bowel \\
\hline B803.00 & Carcinoma in situ of colon \\
\hline B803400 & Carcinoma in situ of caecum \\
\hline B803300 & Carcinoma in situ of sigmoid colon \\
\hline B803700 & Carcinoma in situ of splenic flexure of colon \\
\hline B804000 & Carcinoma in situ of rectosigmoid junction \\
\hline B804100 & Carcinoma in situ of rectum \\
\hline B803600 & Carcinoma in situ of ascending colon \\
\hline $\mathrm{B} 803 \mathrm{zO0}$ & Carcinoma in situ of colon NOS \\
\hline B803100 & Carcinoma in situ of transverse colon \\
\hline $\mathrm{B} 804 \mathrm{zO0}$ & Carcinoma in situ of rectum or rectosigmoid junction NOS \\
\hline B803000 & Carcinoma in situ of hepatic flexure of colon \\
\hline B803200 & Carcinoma in situ of descending colon \\
\hline B804.00 & Carcinoma in situ of rectum and rectosigmoid junction \\
\hline B803800 & High grade dysplasia of colon \\
\hline
\end{tabular}




\subsection{Sensitivity analyses}

\subsubsection{Disease risk score method}

To address residual confounding, we employed a disease risk score (DRS) analysis, used as an alternative to the propensity score method. ${ }^{107} 108$ The DRS was used to estimate the probability of colorectal cancer conditional on being unexposed to GLP-1 analogues and DPP-4 inhibitors. This method involved fitting a Cox proportional hazards model including all potential confounders (listed in the manuscript, Chapter 5) and baseline exposure. Next, the DRS was calculated for each patient as the sum of the products of the regression coefficients and the individual covariate values, setting the exposure status to zero. Stratified on deciles, the DRS was used as a summary statistic in place of all individual potential confounders to estimate HRs of incident colorectal cancer associated with the use of GLP-1 analogues and DPP-4 inhibitors.

\subsubsection{Marginal structural modelling}

We repeated the analysis using a marginal structural Cox proportional hazards model to address potential residual time-dependent confounding over the 9.5-year follow-up period. This method is designed to adjust for time-dependent confounding associated with timevarying exposures. ${ }^{109} 110$ We fit two pooled logistic regression models to estimate the conditional probability of being exposed to GLP-1 analogues and DPP-4 inhibitors given previous treatment history at each 30-day intervals during follow-up; one for the numerator and one for the denominator of the stabilized inverse-probability-of-treatment weights (IPTWs). The numerator model included baseline covariates (listed in the manuscript, Chapter 5) and follow-up time. The denominator model included covariates measured at each time 
interval and follow-up time. In both models, follow-up was modelled using a restricted cubic spline with five knots to reduce bias due to model misspecification from linearity assumptions. ${ }^{111}$ We also estimated inverse probability of censoring weights (IPCWs) in a similar fashion. Stabilized IPTW and IPCW for each patient were computed using the predicted probabilities from both treatment and censoring models. We used the product of these stabilized IPTWs and IPCWs to reweigh the cohort, in which we estimated the HRs of colorectal cancer associated with the use of GLP-1 analogues and DPP-4 inhibitors, with $95 \% \mathrm{Cls}$ calculated using robust variance estimators. ${ }^{110}$

\subsubsection{Multiple imputation}

We repeated the primary analysis using multiple imputation to analyse incomplete data (i.e. missing values for HbA1c, BMI and smoking). ${ }^{112} 113$ This method is more efficient than a complete case analysis. ${ }^{114}$ To impute missing data, an ordinal regression model was used with explanatory variables and cumulative hazard, ${ }^{114}$ as well as the use of GLP-1 analogues or DPP-4 inhibitors at cohort entry. The model was also adjusted for all confounders listed in the manuscript. The results of ten imputations were combined using Rubin's rules. ${ }^{115}$

\subsection{Ethics approval}

This study was approved by the Independent Scientific Advisory Committee of the CPRD, and by the Research Ethics Board of the Jewish General Hospital, Montreal, Canada. 


\section{Chapter 5: Incretin-based drugs and the incidence of colorectal cancer in patients with type 2 diabetes: population-based cohort study}

This chapter presents a manuscript on the association between the use of the incretinbased drugs and incident colorectal cancer. First, background information and study rational is discussed. Next, the methodology outlines the data source, population, covariates and statistical models used. The results provide descriptive characteristics of the cohort, followed by results of the primary and secondary analyses. Finally, the discussion provides a critical analysis of the findings, including comparisons with previous literature, and strengths and limitations of this study. This manuscript has been accepted for publication in Epidemiology. 


\section{Original Research Article}

\section{Incretin-based drugs and the incidence of colorectal cancer in patients with type $\mathbf{2}$ diabetes}

Devin Abrahami ${ }^{1,2}$, Hui Yin ${ }^{1}$, Oriana H Y Yu ${ }^{1,3}$, Michael N Pollak ${ }^{4,5}$, Laurent Azoulay ${ }^{1,4,6}$

${ }^{1}$ Centre for Clinical Epidemiology, Lady Davis Institute, Jewish General Hospital, Montréal, Canada.

${ }^{2}$ Department of Medicine, Division of Experimental Medicine, McGill University, Montréal, Canada.

${ }^{3}$ Division of Endocrinology, Jewish General Hospital, Montreal, Canada.

${ }^{4}$ Gerald Bronfman Department of Oncology, McGill University, Montréal, Canada.

5 Segal Cancer Centre, Lady Davis Institute for Medical Research, Jewish General Hospital, Montreal, Canada

6 Department of Epidemiology, Biostatistics and Occupational Health, McGill University, Montréal, Canada.

Running title: Incretin-based drugs and colorectal cancer

\section{Correspondence:}

Dr Laurent Azoulay

Centre for Clinical Epidemiology, Lady Davis Institute, Jewish General Hospital

3755 Côte Sainte-Catherine, H-425.1

Montreal, Quebec, Canada, H3T 1E2

Tel: 514.340.8222 ext. 28396; Fax: 514.340.7564

Email: laurent.azoulay@mcgill.ca

Conflicts of interest: There is no conflict of interest to declare.

Financial Support: This study was funded by a Foundation Scheme Grant from the Canadian Institutes of Health Research.

Availability of data and code: No additional data are available since it is not permitted according to agreements with the data custodians.

Acknowledgements: Dr. Laurent Azoulay is the recipient of a Chercheur-Boursier career award from the Fonds de recherche du Québec - Santé and a William Dawson Scholar from McGill University. 


\subsection{Abstract}

Background: Evidence on the safety of the incretin-based drugs (glucagon-like peptide-1 [GLP1] analogues and dipeptidylpeptidase-4 [DPP-4] inhibitors) with respect to colorectal cancer is contradictory. The objective of this study was to determine whether use of incretin-based drugs is associated with the risk of incident colorectal cancer in patients with type 2 diabetes.

Methods: Using data from the United Kingdom Clinical Practice Research Datalink, we identified a cohort of 112,040 patients newly- treated with antidiabetic drugs between January 1, 2007 and March 31, 2015. Use of GLP-1 analogues and DPP-4 inhibitors was modelled as a time-varying variable and compared with the use of sulfonylureas. Exposures were lagged by one year for latency and to reduce reverse causality and detection bias. Time-dependent Cox proportional hazards models were used to estimate hazard ratios with $95 \%$ confidence intervals of incident colorectal cancer associated with the use of GLP-1 analogues and DPP-4 inhibitors overall, by cumulative duration of use, and by time since initiation.

Results: During 388,619 person-years of follow-up, there were 733 incident colorectal cancer events (incidence rate: 1.9 per 1,000 person-years). Use of GLP-1 analogues was not associated with colorectal cancer incidence (hazard ratio: 1.04, 95\% confidence interval: $0.68,1.58$ ), nor was use of DPP-4 inhibitors (hazard ratio: 1.19, 95\% confidence interval: $0.95,1.50$ ). There was no evidence of a duration-response relation for either drug.

Conclusions: The results of this large population-based study indicate that use of incretin-based drugs is not associated with colorectal cancer incidence among patients with type 2 diabetes. 


\subsection{Introduction}

Incretin-based drugs, which include glucagon-like peptide-1 (GLP-1) analogues and dipeptidyl peptidase-4 (DPP-4) inhibitors, are used as second-to-third line therapies in the management of type 2 diabetes. ${ }^{1}$ While these drugs lower glucose levels and reduce the risk of hypoglycemia compared to other antidiabetic drugs, ${ }^{2}$ there are concerns that their use may increase the risk of certain malignancies, including colorectal cancer. ${ }^{34}$

The current evidence associating the use of incretin-based drugs with the incidence of colorectal cancer is mixed. According to safety reviews of randomized controlled trials (RCTs) conducted by the US Food and Drug Administration and the European Medicines Agency, no imbalance of colon events was observed with the $1.8 \mathrm{mg}$ formulation of liraglutide (a GLP-1 analogue) compared with placebo. ${ }^{56}$ However, the Food and Drug Administration reported 2 rectal cancer events [0.05\%; 0.06 events per 100 person-years] among users of $1.8 \mathrm{mg}$ liraglutide, compared with no events in the placebo group. ${ }^{5}$ Furthermore, in the Food and Drug Administration's review of the $3 \mathrm{mg}$ formulation of liraglutide (used in weight management), 2 malignant colorectal events were observed in the treatment group [0.06\%; 0.04 events per 100 person-years], while no events were observed in the placebo group. ${ }^{7}$ The evidence continued to be mixed after the publication of large post-marketing RCTs of GLP-1 analogues and DPP-4 inhibitors. ${ }^{8-15}$ While the majority of these RCTs reported no associations with colorectal cancer, ${ }^{8-13}$ saxagliptin (a DPP-4 inhibitor) was associated with a decreased risk of colon cancer (HR: $0.51,95 \% \mathrm{Cl}: 0.27,0.92$ ) in a post-hoc analysis of the SAVOR-TIMI 53 trial. ${ }^{14}$ In contrast, in a pooled analysis of 25 RCTs of sitagliptin (a DPP-4 inhibitor), there was an imbalance in the incidence rate of colon cancer compared with placebo $(0.09 \%$ per year vs $0.04 \%$ per year, 
respectively). ${ }^{15}$ To date, only one observational study was conducted to assess this safety concern and did not observe an association between the use of incretin-based drugs and the incidence of colorectal cancer. ${ }^{16}$

The biological evidence on this potential association is limited, with few studies publishing contradictory findings. On the one hand, there is some evidence that the use of GLP1 analogues and DPP-4 inhibitors may increase the development of malignant colorectal neoplasms. ${ }^{17-19}$ On the other, there is competing evidence that these drugs may have anticancer properties in vitro. ${ }^{20-23}$ Thus, given the discordant information on the association between the use of incretin-based drugs and colorectal cancer incidence, there is an urgent need to assess their safety in the real-world setting. Therefore, the objective of this populationbased study was to determine whether the use of GLP-1 analogues and DPP-4 inhibitors is associated with the incidence of colorectal cancer in patients with type 2 diabetes. 


\subsection{Methods}

\subsubsection{Data source}

This study was conducted using the United Kingdom (UK) Clinical Practice Research Datalink. This database contains anonymized, longitudinal clinical records of over 15 million patients from approximately 700 general practices, and has been shown to be largely representative of the general UK population. ${ }^{24}$ Collected data include information on anthropometric and lifestyle variables, referrals, prescriptions and diagnoses; the data have been shown to be of high quality and validity. ${ }^{25}{ }^{26}$ Furthermore, colorectal cancer diagnoses in the Clinical Practice Research Datalink have been shown to be well recorded when compared with the UK National Cancer Data Repository. ${ }^{27}$

The study protocol was approved by the Independent Scientific Advisory Committee of the Clinical Practice Research Datalink (protocol number 16_264Mn) and by the Research Ethics Board of the Jewish General Hospital, Montreal, Canada.

\subsubsection{Study population}

We identified a base cohort of patients newly-treated with non-insulin antidiabetic drugs (including; metformin, sulfonylureas, prandial glucose regulators, thiazolidinediones, acarbose, DPP-4 inhibitors, GLP-1 analogues, and sodium-glucose co-transporter-2 inhibitors) between January 1, 1988 and March 31, 2015, with follow-up until March 31, 2016. Patients were required to be at least 40 years of age, and with at least one year of medical history in the Clinical Practice Research Datalink before their initial prescription. We excluded patients with advanced type 2 diabetes, identified on the basis of an insulin prescription written before their 
first non-insulin antidiabetic prescription. Female patients with a prior diagnosis of gestational diabetes or polycystic ovary syndrome were also excluded, as these are other indications for metformin.

Using the base cohort, we assembled a study cohort of patients who initiated a new class of an antidiabetic drug in or after 2007 (the year the first incretin-based drugs entered the UK market). ${ }^{2}$ New users included those newly-treated with an antidiabetic drug class (i.e. firstever antidiabetic prescription) as well as those who added-on or switched to an antidiabetic drug class not previously used in their treatment history. Cohort entry was defined by the date of this new antidiabetic drug prescription. We excluded all patients with a history of colorectal cancer (in situ and malignant) and Lynch syndrome ${ }^{28}$ at any time prior to cohort entry, as well as patients with less than one year of follow-up after cohort entry for latency purposes (this included patients diagnosed with colorectal cancer within one year of cohort entry).

All patients meeting the study inclusion criteria were followed starting one year after cohort entry (i.e. person-time at risk) until a first-ever diagnosis of colorectal cancer (in situ and malignant), or censored upon death from any cause, end of registration with the general practice, or the end of the study period (March 31, 2016), whichever occurred first.

\subsubsection{Exposure definition}

Exposure to the different antidiabetic drugs was modelled as a time-varying variable, allowing patients to transition between different exposure groups during the follow-up period. For the GLP-1 analogue analysis, exposure was defined according to the following hierarchical definition: use of GLP-1 analogues (alone or in combination with other antidiabetic drugs), then 
use of sulfonylureas (alone or in combination with other antidiabetic drugs), and finally use of all other antidiabetic drugs. All drug exposures were lagged by one year for latency purposes, and to reduce reverse causality and detection bias. Based on this exposure definition, patients were considered unexposed to the drug of interest until one year after treatment initiation, and considered exposed thereafter for the remainder of follow-up, analogous to an intention-totreat exposure definition. A similar exposure definition was used for the DPP-4 inhibitor analysis, where the following hierarchical exposure definition was used: DPP-4 inhibitors (alone or in combination with other antidiabetic drugs), then use of sulfonylureas (alone or in combination with other antidiabetic drugs), and finally use of all other antidiabetic drugs (Supplementary Figure 1). To avoid confounding by indication, ${ }^{29}$ the reference category for all analyses was the use of sulfonylureas, as these represent an alternative second-to-third line treatment option. We considered, and rejected, the use of metformin as the reference category, as metformin is typically prescribed as a first-line treatment and thus it is not used at the same stage of disease as the incretin-based drugs. ${ }^{30}$

We also defined the use of GLP-1 analogues and DPP-4 inhibitors in terms of cumulative duration of use and time since initiation, which were modeled as time-dependent variables. Cumulative duration of use was calculated by summing the durations associated with each prescription from cohort entry until the time of event (risk set). Time since initiation was defined as the time between the first ever prescription of a GLP-1 analogue or DPP-4 inhibitor and the risk set date. 


\subsubsection{Potential confounders}

The models were adjusted for the following potential confounders measured at cohort entry: age, sex, year of cohort entry, alcohol-related disorders (including alcoholism, alcoholic cirrhosis of the liver, alcoholic hepatitis and hepatic flexure), smoking status (current, former, never, unknown), body mass index (BMI) $\left(<25 \mathrm{~kg} / \mathrm{m}^{2}, 25-29 \mathrm{~kg} / \mathrm{m}^{2}, \geq 30 \mathrm{~kg} / \mathrm{m}^{2}\right.$, unknown), hemoglobin A1c (last laboratory result before cohort entry), duration of treated diabetes (defined as the time between first non-insulin prescription and cohort entry), previous cancer (other than non-melanoma skin cancer), inflammatory bowel disease (Crohn's disease and ulcerative colitis) and Charlson comorbidity score. We also adjusted the models for the presence of microvascular complications of diabetes (neuropathy, renal disease, retinopathy, and peripheral arteriopathy; measured at any time before cohort entry) and the number of unique antidiabetic drugs received in the year before cohort entry, both as proxies for diabetes severity. Models were adjusted for the total number of unique non-antidiabetic drugs in the year before cohort entry, as a general measure of comorbidity. ${ }^{31}$ Finally, models were adjusted for use of aspirin and statins at any time before cohort entry, as these drugs have been associated with a decreased risk of colorectal cancer in some studies. ${ }^{32} 33$

\subsubsection{Statistical analysis}

Crude incidence rates of colorectal cancer were calculated for the entire cohort, and for each exposure group. For the primary analysis, time-dependent Cox proportional hazards models were used to estimate hazard ratios (HRs) and 95\% confidence intervals (Cls) of incident colorectal cancer associated with the overall use of GLP-1 analogues and DPP-4 inhibitors 
compared with the use of sulfonylureas. All models were adjusted for the potential confounders listed previously.

\subsubsection{Secondary analyses}

We conducted four secondary analyses. First, we assessed whether there was a duration-response relation for GLP-1 analogue and DPP-4 inhibitor cumulative duration of use on the incidence of colorectal cancer. For this time-dependent analysis, HRs were estimated for three predefined duration categories: $\leq 1$ year, $1.1-2$ years and $>2$ years. Second, we assessed the association between time since initiation of GLP-1 analogues and DPP-4 inhibitors and colorectal cancer incidence ( $\leq 2$ years and $>2$ years). Third, we assessed the association with the most common individual drugs within each incretin-based drug class (GLP-1 analogues: exenatide and liraglutide; DPP-4 inhibitors: sitagliptin, saxagliptin). Finally, the analyses were repeated after stratifying on colon versus rectal cancer.

\subsubsection{Sensitivity analyses}

We conducted seven sensitivity analyses to assess the robustness of our findings. First, we repeated the primary analysis after increasing the exposure lag period to two years, as there are uncertainties related to the length of the latency time window. Conversely, to explore the possibility that the previously reported increased risk was due to a tumor promoter effect, we repeated the analyses by removing the lag period. Third, to assess possible detection bias of undiagnosed colorectal cancer, we stratified the cohort based on referrals to colonoscopy screening or fecal occult blood testing, measured in the 5 years before cohort entry. ${ }^{34}$ Fourth, 
to address the possibility of outcome misclassification, we repeated the analysis upon restricting to malignant colorectal cancer and censoring on in situ colorectal cancer diagnoses. In the last three sensitivity analyses, we addressed possible residual confounding by repeating the primary analyses using the disease risk score method, marginal structural models, and multiple imputation for variables with missing information (Chapter 4). All analyses were conducted with SAS version 9.4 (SAS institute, Cary, NC). 


\subsection{Results}

A total of 112,040 patients met the study inclusion criteria (Figure 6), and were followed for a mean (standard deviation [SD]) of 3.5 (2.2) years after completing the one-year postcohort entry latency period. During 388,619 person-years of follow-up, there were 733 incident colorectal cancer events, generating a crude incidence rate of $1.4(95 \% \mathrm{Cl}: 1.3,1.5)$ per 1000 person-years. Among these events, 715 (incidence rate 1.8, 95\% Cl: 1.7, 2.0 per 1000 personyears) were malignant versus 18 (incidence rate $0.05,95 \% \mathrm{Cl}: 0.03,0.07$ per 1000 person-years) in situ colorectal cancers. A total of 5724 (5.1\%) patients were prescribed GLP-1 analogues during the study period, and 22,276 (19.9\%) patients were prescribed DPP-4 inhibitors.

Table 5 presents baseline characteristics for the cohort overall, and stratified by use of GLP-1 analogues, DPP-4 inhibitors, and sulfonylureas at cohort entry. Compared with sulfonylurea users, GLP-1 analogue users were younger, more likely to have had alcohol-related disorders, and less likely to be current smokers. Additionally, GLP-1 analogue users were more likely to have a higher BMI, to have a higher hemoglobin A1c level, and were more likely to have neuropathy and retinopathy. Compared with sulfonylurea users, DPP-4 inhibitor users were older, more likely to have had alcohol-related disorders, and less likely to be current smokers. DPP-4 inhibitor users were more likely to have a higher BMI, to have a higher hemoglobin A1c level and a higher Charlson comorbidity score. DPP-4 inhibitor users were also more likely to have neuropathy and retinopathy.

The results of primary and secondary analyses are shown in Tables 6 and 7. Compared with the use of sulfonylureas, the use of GLP-1 analogues was not associated with the incidence of colorectal cancer (2.0 vs 1.6 per 1000 per year, respectively; HR: $1.04 .95 \% \mathrm{Cl}: 0.68,1.58)$. 
Furthermore, there was no evidence of a duration-response relation both in terms of cumulative duration of use ( $\leq 1$ year, HR: $0.68,95 \%$ : $0.32,1.46$; $1.1-2$ years, HR: $1.44,95 \% \mathrm{Cl}$ : $0.80,2.60$; > 2 years, HR: $1.08,95 \% \mathrm{Cl}: 0.50,2.31$ ) and time since initiation ( $\leq 2$ years, HR: 1.22 , 95\% Cl: 0.62, 2.40; > 2 years, HR: 0.96, 95\% Cl: 0.58, 1.59) (Table 6).

Compared with the use of sulfonylureas, the use of DPP-4 inhibitors was not associated with the incidence of colorectal cancer overall (1.9 vs 2.1 per 1000 per year, respectively; HR: $1.19,95 \% \mathrm{Cl}: 0.95,1.50)$, or by cumulative duration of use ( $\leq 1$ year, HR: $1.42,95 \% \mathrm{Cl}: 1.00$, 2.01; $1.1-2$ years, HR: $1.00,95 \% \mathrm{Cl}: 0.70,1.45 ;>2$ years, HR: $1.20,95 \% \mathrm{Cl}: 0.87,1.67)$, and time since initiation ( $\leq 2$ years, HR: $1.34,95 \%$ : 0.96, 1.86; > 2 years, HR: $1.12,95 \% \mathrm{Cl}: 0.85,1.47$ ) (Table 7). Similar findings were observed when analyses were repeated stratifying on individual drug type and when stratifying on colon versus rectal cancer (Supplementary Tables 1 to 4).

\subsubsection{Sensitivity analyses}

The results of the sensitivity analyses were consistent with those of the primary analysis (Supplementary Figure 2 and Supplementary Tables 5 to 18). For GLP-1 analogues, the adjusted HRs ranged between 0.91 and 1.85, while for DPP-4 inhibitors, the adjusted HRs ranged between 0.84 and 1.20 . 


\subsection{Discussion}

In this large population based cohort study with a mean follow-up of 3.5 years (after accounting for a one-year post-cohort entry latency period) and with up to 9.5 years of potential follow-up, the use of GLP-1 analogues and DPP-4 inhibitors was not associated with incident colorectal cancer, when compared with the use of sulfonylureas. Furthermore, there were no associations by cumulative duration of use or time since initiation and the findings remained highly consistent in sensitivity analyses that considered different sources of bias.

Overall, our findings are consistent with the vast majority of RCTs which showed no association between different incretin-based drugs and colorectal cancer incidence. ${ }^{8-13}$ Moreover, our findings provide some reassurance that the diabetic dose of liraglutide $(1.8 \mathrm{mg}$ ) is not associated with an increased risk of colorectal cancer. However, it is not possible to rule out a potential increased risk of colorectal cancer with higher doses of liraglutide, such as those used in weight management trials. Indeed, our study population did not include users of the 3 mg formulation, which is commonly used for treatment of obesity among non-diabetic patients. With respect to DPP-4 inhibitors, we did not observe any association with colorectal cancer incidence either overall or by individual drug types. This is inconsistent with prior RCTs that produced contradictory evidence; both decreased ${ }^{14}$ and increased incidences ${ }^{15}$ have been reported with saxagliptin and sitagliptin, respectively. However, these RCTs were not designed or powered to assess cancer incidence, and thus generated few events limiting the interpretation of their findings.

To our knowledge, only one observational study has been conducted to assess this possible association. ${ }^{16}$ Using US Medicare data from 2007 to 2013 and an as-treated exposure 
definition, there was no association between the use of DPP-4 inhibitors and incident colorectal cancer, regardless of whether these drugs were compared to thiazolidinediones or sulfonylureas (HR: $1.17,95 \% \mathrm{Cl}: 0.88,1.71$ and $\mathrm{HR}: 0.98,95 \% \mathrm{Cl}: 0.74,1.30$, respectively). ${ }^{16}$ Similar findings were observed for the use of GLP-1 analogues, when compared with the use of long-acting insulin (HR: $0.82,95 \% \mathrm{Cl}: 0.42,1.58) .{ }^{16}$ While our findings are confirmatory of the previous study, ${ }^{16}$ the latter was limited by its short duration of follow-up, which ranged between 0.7 and 1.2 years. Such short durations limit the interpretation of safety for outcomes such as colorectal cancer. ${ }^{8-16}$

The existing biological evidence on the relation between the use of incretin-based drugs and colorectal cancer is contradictory. ${ }^{17-23}$ While our study did not find an increase in the incidence of colorectal cancer with these drugs, it is not possible to completely rule out a tumor promoter effect with the use of GLP-1 analogues. Indeed, GLP-1 analogues may enhance the growth of the small and large bowel via fibroblast growth factor 7 (FGF7), and activation of the GLP-1 signaling pathway may promote gut growth and crypt fission. ${ }^{17}$ This effect may be masked by the possible anti-cancer properties of GLP-1 analogues observed in vitro, whereby increased activation of the GLP-1 receptor has been shown to alter cell morphology, induce apoptosis, and inhibit proliferation of colon cancer cells. ${ }^{2023}$

The evidence on DPP-4 inhibitors is lacking; these drugs have been shown to be cytotoxic agents against colon carcinoma cells and lower colon carcinogenesis in rat models. ${ }^{2122}$ However, there is competing evidence that long-term inhibition of the DPP-4 enzyme can lead to immune dysregulation and increased cancer risk, ${ }^{18}$ and that DPP-4 inhibitor use may support the metastasis of colon cancer cells. ${ }^{19}$ Overall, such experimental studies should be interpreted 
with caution, as animal models do not represent the complex pathophysiology of patients with type 2 diabetes, a population that is already at an increased risk of colorectal cancer. ${ }^{35}$ Overall, our findings suggest that the incretin-based drugs are likely to have neutral effects on colorectal carcinogenesis in the relative short-term, and thus future studies with longer follow-up will be needed to confirm our findings.

This study has several strengths. First, our study generated 388,619 person-years of follow-up, allowing for the identification of a substantial number of incident colorectal cancer events. Second, we used a new-user study design to reduce biases associated with the inclusion of prevalent users. ${ }^{36}$ Third, we used a time-dependent exposure definition that took into account the dynamic nature of the pharmacological management of type 2 diabetes, while eliminating the possibility of immortal time bias. ${ }^{37}$ Fourth, a lag period was used for latency purposes and to reduce detection bias and reverse causality. Fifth, all models were adjusted for a number of potential confounders including smoking status, alcohol-related disorders, and $\mathrm{BMI}$, which are all known risk factors of colorectal cancer. Finally, the results remained consistent across several sensitivity analyses, illustrating the robustness of our findings.

This study also has some limitations. Some exposure misclassification is possible as prescriptions in the Clinical Practice Research Datalink represent those written by general practitioners and not specialists. However, in the UK, general practitioners are responsible for maintaining the long-term care of patients with type 2 diabetes, and thus we expect such misclassification to have had an unimportant impact on our exposure definition. Although colorectal cancer diagnoses have been shown to be well recorded in the Clinical Practice Research Datalink, ${ }^{27}$ outcome misclassification remains possible. However, we expect this 
potential misclassification to be non-differential between the treatment groups. As with all observational studies, residual confounding from unmeasured variables (such as family history or race/ethnicity) is possible, although it is unclear how these unmeasured variables would influence the prescribing of incretin-based drugs. Furthermore, we obtained consistent results in sensitivity analyses using the disease risk score method and marginal structural models to control for time-dependent confounding. This study is also limited by its mean follow-up of 3.5 years after accounting for a one-year latency after cohort entry, which may be considered short to assess cancer incidence. However, the rational for conducting this study was based on signals from short duration RCTs (12 weeks to 2.1 years), ${ }^{14}{ }^{15}$ and thus our study had long enough follow-up to assess colorectal cancer incidence in the relative short-term. Furthermore, based on the upper limits of the Cls of the main analyses (1.58 for use of GLP-1 analogues and 1.50 for use of DPP-4 inhibitors), our study was sufficiently powered to rule out strong associations between the use of the incretin-based drugs and colorectal cancer; though the possibility of weaker associations remains possible. Finally, given the rarity of the outcome, some of the secondary analyses resulted in wide Cls.

In summary, the results of this large population-based study indicate that compared with the use of sulfonylureas, the use of incretin-based drugs is not associated with a substantial increase in the incidence, and may be unassociated with the incidence of colorectal cancer, among patients with type 2 diabetes. 


\subsection{References}

1. Drucker DJ, Nauck MA. The incretin system: glucagon-like peptide-1 receptor agonists and dipeptidyl peptidase-4 inhibitors in type 2 diabetes. The Lancet 2006;368(9548):1696705.

2. Nauck MA, Vilsbøll T, Gallwitz B, et al. Incretin-Based Therapies Viewpoints on the way to consensus. Diabetes Care 2009;32(suppl 2):S223-S31.

3. Halfdanarson TR, Pannala R. Incretins and risk of neoplasia. BMJ : British Medical Journal 2013;346.

4. Tseng C-H, Lee K-Y, Tseng F-H. An Updated Review on Cancer Risk Associated with Incretin Mimetics and Enhancers. Journal of Environmental Science and Health, Part C 2015;33(1):67-124.

5. Liraglutide (injection) for the Treatment of Patients with Type 2 Diabetes Food and Drug Administration.

6. Assessment Report for Victoza. European Medicines Agency.

7. Summary Review for Regulatory Action SAXENDA (liraglutide [rDNA origin] injection)

8. Green JB, Bethel MA, Armstrong PW, et al. Effect of sitagliptin on cardiovascular outcomes in type 2 diabetes. N Engl J Med 2015;373(3):232-42.

9. Marso SP, Daniels GH, Brown-Frandsen K, et al. Liraglutide and cardiovascular outcomes in type 2 diabetes. N Engl J Med 2016;375(4):311-22.

10. Pi-Sunyer X, Astrup A, Fujioka K, et al. A randomized, controlled trial of $3.0 \mathrm{mg}$ of liraglutide in weight management. N Engl J Med 2015;373(1):11-22. 
11. White WB, Cannon CP, Heller SR, et al. Alogliptin after acute coronary syndrome in patients with type 2 diabetes. N Engl J Med 2013;369(14):1327-35.

12. Marso SP, Bain SC, Consoli A, et al. Semaglutide and Cardiovascular Outcomes in Patients with Type 2 Diabetes. N Engl J Med 2016;375(19):1834-44.

13. Pfeffer MA, Claggett B, Diaz R, et al. Lixisenatide in Patients with Type 2 Diabetes and Acute Coronary Syndrome. N Engl J Med 2015;373(23):2247-57.

14. Leiter L, Teoh H, Mosenzon O, et al. Frequency of cancer events with saxagliptin in the SAVOR-TIMI 53 trial. Diabetes, Obesity and Metabolism 2016;18(2):186-90.

15. Engel SS, Round E, Golm GT, et al. Safety and tolerability of sitagliptin in type 2 diabetes: pooled analysis of 25 clinical studies. Diabetes Ther 2013;4(1):119-45.

16. Htoo PT, Buse JB, Gokhale M, et al. Effect of glucagon-like peptide-1 receptor agonists and dipeptidyl peptidase-4 inhibitors on colorectal cancer incidence and its precursors. Eur J Clin Pharmacol 2016;72(8):1013-23.

17. Koehler JA, Baggio LL, Yusta B, et al. GLP-1R agonists promote normal and neoplastic intestinal growth through mechanisms requiring Fgf7. Cell Metab 2015;21(3):379-91.

18. Stulc T, Sedo A. Inhibition of multifunctional dipeptidyl peptidase-IV: Is there a risk of oncological and immunological adverse effects? Diabetes Res Clin Pract 2010;88(2):12531.

19. Masur K, Schwartz F, Entschladen F, et al. DPPIV inhibitors extend GLP-2 mediated tumour promoting effects on intestinal cancer cells. Regul Pept 2006;137(3):147-55. 
20. Koehler JA, Kain T, Drucker DJ. Glucagon-like peptide-1 receptor activation inhibits growth and augments apoptosis in murine CT26 colon cancer cells. Endocrinology 2011;152(9):3362-72.

21. Amritha C, KumArAvelu P, ChellAthAi DD. Evaluation of Anti Cancer Effects of DPP-4 Inhibitors in Colon Cancer-An Invitro Study. Journal of clinical and diagnostic research: JCDR 2015;9(12):FC14.

22. Femia AP, Raimondi L, Maglieri G, et al. Long-term treatment with Sitagliptin, a dipeptidyl peptidase-4 inhibitor, reduces colon carcinogenesis and reactive oxygen species in 1,2dimethylhydrazine-induced rats. Int J Cancer 2013;133(10):2498-503.

23. Tawfik MK, Mohamed MI. Exenatide suppresses 1,2-dimethylhydrazine-induced colon cancer in diabetic mice: Effect on tumor angiogenesis and cell proliferation. Biomed Pharmacother 2016;82:106-16.

24. Herrett E, Gallagher AM, Bhaskaran K, et al. Data Resource Profile: Clinical Practice Research Datalink (CPRD). Int J Epidemiol 2015;44(3):827-36.

25. Jick SS, Kaye JA, Vasilakis-Scaramozza C, et al. Validity of the general practice research database. Pharmacotherapy 2003;23(5):686-9.

26. Lawrenson R, Williams T, Farmer R. Clinical information for research; the use of general practice databases. J Public Health Med 1999;21(3):299-304.

27. Boggon R, Staa TP, Chapman M, et al. Cancer recording and mortality in the General Practice Research Database and linked cancer registries. Pharmacoepidemiol Drug Saf 2013;22(2):168-75. 
28. Lynch H, Lynch P, Lanspa S, et al. Review of the Lynch syndrome: history, molecular genetics, screening, differential diagnosis, and medicolegal ramifications. Clin Genet $2009 ; 76(1): 1-18$

29. Suissa S, Azoulay L. Metformin and the Risk of Cancer: Time-related biases in observational studies. Diabetes Care 2012;35(12):2665-73.

30. Raz I. Guideline approach to therapy in patients with newly diagnosed type 2 diabetes. Diabetes Care 2013;36(Supplement 2):S139-S44.

31. Schneeweiss S, Seeger JD, Maclure M, et al. Performance of comorbidity scores to control for confounding in epidemiologic studies using claims data. Am J Epidemiol 2001;154(9):854-64.

32. Garcia-Albeniz X. Aspirin for the prevention of colorectal cancer. 2011;25(0):461-72.

33. Liu Y, Tang W, Wang J, et al. Association between statin use and colorectal cancer risk: a meta-analysis of 42 studies. Cancer Causes Control 2014;25(2):237-49.

34. Public Health England. NHS bowel cancer screening (BCSP) programme. Secondary Public Health England. NHS bowel cancer screening (BCSP) programme. https://www.gov.uk/topic/population-screening-programmes/bowel

35. Berster JM, Göke B. Type 2 diabetes mellitus as risk factor for colorectal cancer. Arch Physiol Biochem 2008;114(1):84-98.

36. Ray WA. Evaluating medication effects outside of clinical trials: new-user designs. Am J Epidemiol 2003;158(9):915-20.

37. Suissa S. Immortal time bias in pharmaco-epidemiology. Am J Epidemiol 2008;167(4):492-9. 


\subsection{Figures and tables}

FIGURE LEGEND

Figure 6 Study flow chart of patients included in the base and study cohorts. 


\section{Figure 6. Study flow chart}

\begin{tabular}{|c|c|c|c|c|}
\hline \multirow[t]{2}{*}{384,772} & \multicolumn{3}{|c|}{$\begin{array}{l}\text { Patients with a first-ever } \\
\text { prescription for a non-insulin anti- } \\
\text { diabetic drug between January 1, } \\
1988 \text { to March 31, } 2015\end{array}$} & \\
\hline & & 198,278 & $\begin{array}{l}\text { Excluded } \\
\begin{array}{l}39,628 \\
149,530 \\
57 \\
6484 \\
1872 \\
707\end{array}\end{array}$ & $\begin{array}{l}<40 \text { years of age } \\
<365 \text { days coverage in the database } \\
\text { Date inconsistencies } \\
\text { Insulin before first-ever non-insulin anti-diabetic drug } \\
\text { Women with diagnosed polycystic ovarian syndrome } \\
\text { Women with gestational diabetes in the year before first prescription }\end{array}$ \\
\hline \multirow[t]{2}{*}{186,494} & \multicolumn{2}{|c|}{$\begin{array}{l}\text { Patients included in the base- } \\
\text { cohort }\end{array}$} & & \\
\hline & - & 58,142 & $\begin{array}{l}\text { Excluded } \\
\mathbf{2 3 , 9 6 3} \\
\mathbf{3 4 , 1 7 9}\end{array}$ & $\begin{array}{l}\text { Died or left cohort before first incretin-based drug } \\
\text { entered the market } \\
\text { Never added-on or switched to new anti-diabetic drug } \\
\text { class after incretin-based drugs entered the market }\end{array}$ \\
\hline \multirow[t]{2}{*}{128,352} & \multicolumn{2}{|c|}{$\begin{array}{l}\text { Cohort of new-users or } \\
\text { switchers after incretin-based } \\
\text { drugs entered the market }\end{array}$} & & \\
\hline & & 16,312 & $\begin{array}{l}\text { Excluded } \\
0 \\
1468 \\
14,844\end{array}$ & $\begin{array}{l}\text { Previous lynch syndrome } \\
\text { Previous colorectal cancer } \\
\text { Less than } 1 \text { year of follow-up }\end{array}$ \\
\hline 112,040 & Study cohort & & & \\
\hline
\end{tabular}


Table 5. Baseline Demographic and Clinical Characteristics of the Cohort and Stratified by Drug Use at Cohort Entry

Use at Cohort Entry ${ }^{\text {a }}$

\section{Characteristic}

Total
Age, years (mean, SD)

Male, n (\%)

Year of cohort entry, $\mathrm{n}(\%)$

2007

2008

2009

2010

2011

2012

2013

2014

2015

Alcohol-related disorders, $\mathrm{n}(\%)$

Smoking status, $\mathrm{n}(\%)$

Current

Past

Never

Unknown

Body mass index, $\mathrm{n}(\%)$

$<25 \mathrm{~kg} / \mathrm{m}^{2}$

$25-30 \mathrm{~kg} / \mathrm{m}^{2}$

$\geq 30.0$

Unknown

Hemoglobin A1c, n (\%)

$\leq 7.0 \%$

$7.1 \%-8.0 \%$

$>8.0 \%$

Unknown

Duration of treated diabetes in years (mean, SD)

Cancer, $\mathrm{n}$ (\%)

Inflammatory bowel disease, $\mathrm{n}(\%)$

Charlson comorbidity score

0

$1-2$

$\geq 3$

Neuropathy, n (\%)

Renal disease, $\mathrm{n}(\%)$

Retinopathy, $\mathrm{n}(\%)$

Peripheral arteriopathy, $\mathrm{n}(\%)$

Aspirin use, $\mathrm{n}(\%)$

Statins use, $n(\%)$

Number of non-antidiabetic drugs, $\mathrm{n}(\%)$

Entire Cohort GLP-1 analogues DPP-4 inhibitors Sulfonylureas

\begin{tabular}{|c|c|c|c|}
\hline 112,040 & 1177 & 6002 & 18,513 \\
\hline $63.6(12)$ & $60.0(8.1)$ & $66.9(10)$ & $65.9(12)$ \\
\hline $65,166(58)$ & $666(57)$ & 3465 (58) & 10,953 (59) \\
\hline $18,439(17)$ & $51(4.3)$ & 115 (1.9) & $4362(24)$ \\
\hline $16,820(15)$ & $229(20)$ & 476 (7.9) & 3715 (20) \\
\hline $16,944(15)$ & $305(26)$ & $947(16)$ & 3076 (17) \\
\hline $15,696(14)$ & $257(22)$ & $1385(23)$ & 2347 (13) \\
\hline $13,097(12)$ & $153(13)$ & 1022 (17) & $1807(9.8)$ \\
\hline $11,741(11)$ & 105 (9) & $880(15)$ & $1362(7.4)$ \\
\hline $10,102(9.0)$ & $47(4.0)$ & 646 (11) & $1007(5.4)$ \\
\hline 7805 (7.0) & $\mathrm{S}^{\mathrm{b}}$ & $452(7.5)$ & 735 (4.0) \\
\hline $1396(1.2)$ & $\mathrm{S}^{\mathrm{b}}$ & 79 (1.3) & $102(0.5)$ \\
\hline $16,329(15)$ & $221(20)$ & 1158 (19) & 2775 (15) \\
\hline $17,183(15)$ & $142(12)$ & $758(13)$ & 2741 (15) \\
\hline 42,659 (38) & $\mathrm{S}^{\mathrm{b}}$ & $\mathrm{S}^{\mathrm{b}}$ & 7129 (39) \\
\hline $51,899(46)$ & $511(43)$ & $2788(47)$ & $8573(46)$ \\
\hline $299(0.3)$ & $\mathrm{S}^{\mathrm{b}}$ & $S^{b}$ & $70(0.4)$ \\
\hline $11,384(10)$ & $9(0.8)$ & $576(9.6)$ & 3183 (17) \\
\hline $33,976(30)$ & $81(6.9)$ & $1809(30)$ & $6216(34)$ \\
\hline $64,582(58)$ & $1087(92)$ & $3601(60)$ & 8691 (47) \\
\hline 2098 (1.9) & $0(0.0)$ & $16(0.3)$ & $423(2.3)$ \\
\hline $17,187(15)$ & $147(13)$ & $674(11)$ & $1972(11)$ \\
\hline $31,439(28)$ & $208(18)$ & $1941(32)$ & 4995 (27) \\
\hline $47,866(43)$ & 811 (69) & $3333(56)$ & $9298(50)$ \\
\hline $15,548(14)$ & $11(0.9)$ & $54(0.9)$ & $2248(12)$ \\
\hline $1.4(3.0)$ & $8.0(3.9)$ & 7.7 (3.9) & $2.9(3.2)$ \\
\hline $11,770(11)$ & $103(8.8)$ & 756 (13) & $2293(12)$ \\
\hline $1350(1.2)$ & $16(1.4)$ & $87(1.4)$ & $302(1.6)$ \\
\hline $42,139(38)$ & $309(26)$ & 1036 (17) & $6075(33)$ \\
\hline $52,390(47)$ & $603(51)$ & 3207 (53) & 8349 (45) \\
\hline $17,511(16)$ & $265(23)$ & 1759 (29) & $4089(22)$ \\
\hline $12,235(11)$ & $320(27)$ & $1528(26)$ & $2778(15)$ \\
\hline $18,438(17)$ & $249(21)$ & $1613(27)$ & $4583(25)$ \\
\hline $12,562(11)$ & $325(28)$ & $1881(31)$ & 2787 (15) \\
\hline 4365 (3.9) & $52(4.4)$ & $358(6.0)$ & $921(5.0)$ \\
\hline $52,018(46)$ & $808(69)$ & $4076(68)$ & $10,358(56)$ \\
\hline $80,644(72)$ & $1072(91)$ & 5464 (91) & 14,177 (77) \\
\hline
\end{tabular}




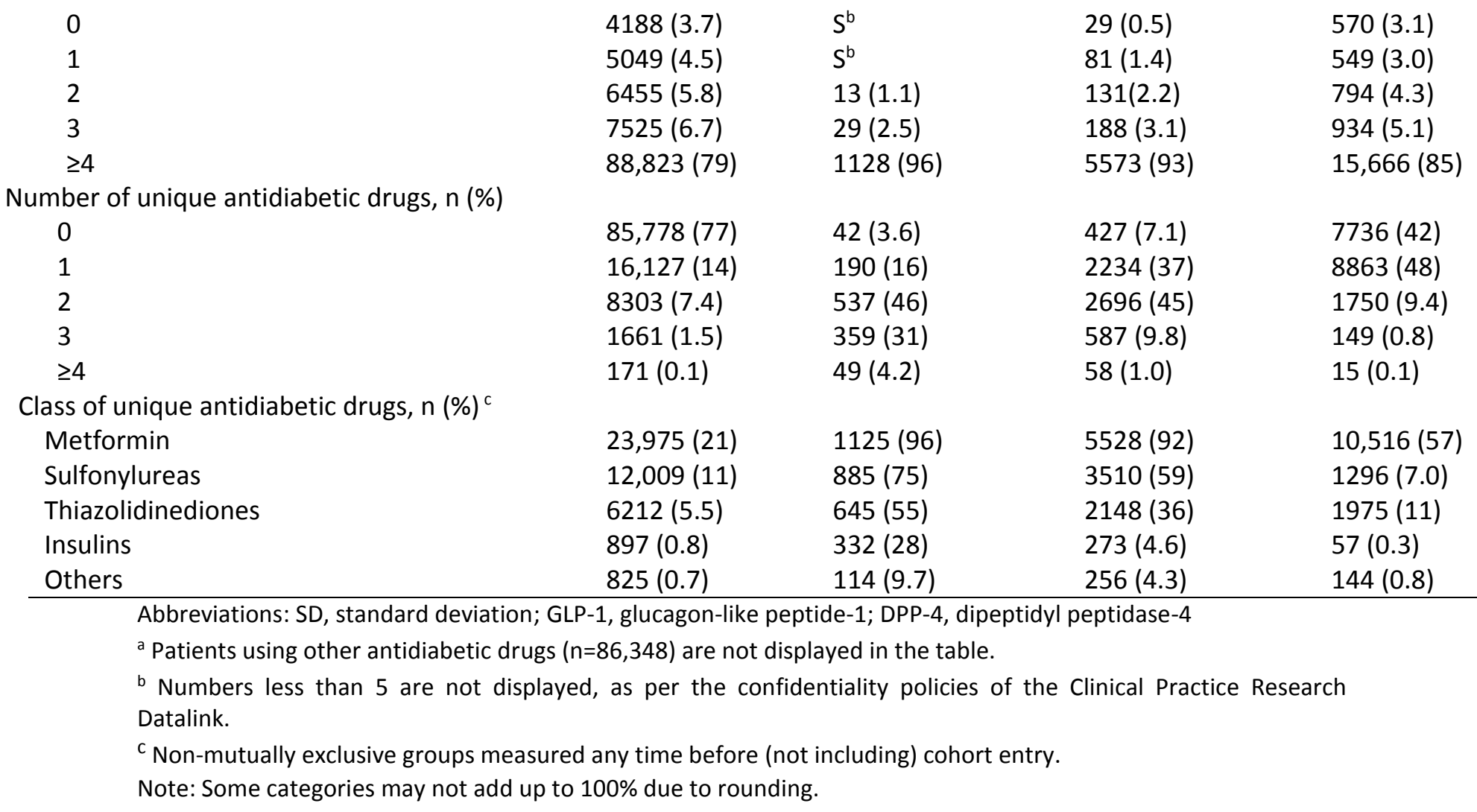




\begin{tabular}{|c|c|c|c|c|c|}
\hline Exposure $^{a}$ & Events & Person-years & Incidence rate ${ }^{b}(95 \% \mathrm{Cl})$ & Crude HR & Adjusted HR $(95 \% \mathrm{Cl})^{\mathrm{c}}$ \\
\hline Sulfonylureas & 302 & 151,949 & $1.99(1.77,2.22)$ & 1.00 & 1.00 [Reference] \\
\hline GLP-1 analogues & 26 & 16,135 & $1.61(1.05,2.36)$ & 0.78 & $1.04(0.68,1.58)$ \\
\hline \multicolumn{6}{|c|}{ Duration of GLP-1 analogue use, years } \\
\hline$\leq 1$ & 7 & 6504 & $1.08(0.43,2.22)$ & 0.53 & $0.68(0.32,1.46)$ \\
\hline $1.1-2$ & 12 & 5610 & $2.14(1.11,3.74)$ & 1.05 & $1.44(0.80,2.60)$ \\
\hline$>2$ & 7 & 4021 & $1.74(0.70,3.59)$ & 0.82 & $1.08(0.50,2.31)$ \\
\hline \multicolumn{6}{|c|}{ Time since first GLP-1 analogue use, years } \\
\hline$\leq 2$ & 9 & 5177 & $1.74(0.79,3.30)$ & 0.89 & $1.22(0.62,2.40)$ \\
\hline$>2$ & 17 & 10,958 & $1.55(0.90,2.48)$ & 0.73 & $0.96(0.58,1.59)$ \\
\hline
\end{tabular}

Abbreviations: HR, hazard ratio; $\mathrm{Cl}$, confidence interval; GLP-1, glucagon-like peptide-1

a Use of other antidiabetic drugs was considered in the model, but not presented in the table.

b Per 1000 Person-Years.

' Adjusted for age, sex, year of cohort entry, body mass index (BMI), smoking, alcohol-related disorders (including for example alcoholism, alcoholic cirrhosis of the liver, alcoholic hepatitis and hepatic flexure), hemoglobin A1c, duration of treated diabetes, previous cancer, presence of inflammatory bowel disease,

Charlson comorbidity score, neuropathy, renal disease, retinopathy, peripheral arteriopathy, aspirin, statins, the number of unique antidiabetic drugs and the total number of unique non-diabetic drugs in the year before cohort entry. 
Table 7. Crude and Adjusted HRs for the Association Between the Use of DPP-4 Inhibitors and the Risk of Colorectal Cancer

\begin{tabular}{|c|c|c|c|c|c|}
\hline Exposure $^{a}$ & Events & Person-years & Incidence rate ${ }^{b}(95 \% \mathrm{Cl})$ & Crude HR & Adjusted HR $(95 \% \mathrm{Cl})^{\mathrm{c}}$ \\
\hline Sulfonylureas & 241 & 127,443 & $1.89(1.66,2.15)$ & 1.00 & 1.00 [Reference] \\
\hline DPP-4 inhibitors & 117 & 56,613 & $2.07(1.71,2.48)$ & 1.06 & $1.19(0.95,1.50)$ \\
\hline \multicolumn{6}{|c|}{ Duration of DPP-4 inhibitor use, years } \\
\hline$\leq 1$ & 38 & 16,248 & $2.34(1.66,3.21)$ & 1.22 & $1.42(1.00,2.01)$ \\
\hline $1.1-2$ & 34 & 19,820 & $1.72(1.19,2.40)$ & 0.90 & $1.00(0.70,1.45)$ \\
\hline$>2$ & 45 & 20,545 & $2.19(1.60,2.93)$ & 1.10 & $1.20(0.87,1.67)$ \\
\hline \multicolumn{6}{|c|}{ Time since first DPP-4 inhibitor use, years } \\
\hline$\leq 2$ & 43 & 19,625 & $2.19(1.59,2.95)$ & 1.19 & $1.34(0.96,1.86)$ \\
\hline$>2$ & 74 & 36,988 & $2.00(1.57,2.51)$ & 1.00 & $1.12(0.85,1.47)$ \\
\hline
\end{tabular}

Abbreviations: HR, hazard ratio; $\mathrm{Cl}$, confidence interval; DPP-4, dipeptidyl peptidase-4

a Use of other anti-diabetic drugs was considered in the model, but not presented in the table.

b Per 1000 Person-Years.

c Adjusted for age, sex, year of cohort entry, body mass index (BMI), smoking, alcohol-related disorders (including for example alcoholism, alcoholic cirrhosis of the liver, alcoholic hepatitis and hepatic flexure), hemoglobin A1c, duration of treated diabetes, previous cancer, presence of inflammatory bowel disease,

Charlson comorbidity score, neuropathy, renal disease, retinopathy, peripheral arteriopathy, aspirin, statins, the number of unique antidiabetic drugs and the total number of unique non-diabetic drugs in the year before cohort entry.

haemoglobin A1c, duration of treated diabetes, previous cancer, presence of inflammatory bowel disease, Charlson comorbidity score, neuropathy, renal disease, retinopathy, peripheral arteriopathy, the number of unique antidiabetic drugs and the total number of unique non-diabetic drugs in the year before cohort entry. 
FIGURE LEGENDS

Supplementary Figure 1 Summary of the hierarchical exposure definition. The dashed lines represent the one-year lag period after each new antidiabetic prescription; patients were considered exposed to each new antidiabetic drug starting 1 year after initial prescription. The dotted line (in green) represents the hierarchical nature of the exposure definition, whereby patients were considered exposed to GLP-1 analogues (or DPP-4 inhibitors) until the end of followup, regardless of treatment discontinuation, switch or add-on of other antidiabetic drugs. Finally, each event date formed a risk set, where exposure to the different antidiabetic drugs was assessed at these time points.

In the scenario above, Patient A experienced the outcome after one year of follow-up (Risk set 1). At that point in time, this patient's exposure is compared with the exposure of Patient B (sulfonylurea), Patient C (GLP-1 analogue), Patient D (other) and Patient E (GLP-1 analogue). At risk set 3, Patient C, exposed to GLP-1 analogue, is compared to Patient D (sulfonylurea) and Patient E (GLP-1 analogue). The latter discontinued the use of a GLP-1 analogue at year 2 of the follow-up, but was considered exposed until the end of the follow-up.

Supplementary Figure 2 Forest plot summarizing the results of the primary analysis and sensitivity analyses. The results are displayed as adjusted hazard ratios and $95 \%$ confidence intervals for the association between the use of GLP-1 analogues and DPP-4 inhibitors and incident colorectal cancer. The reference category for all analyses was the use of sulfonylureas. 
Supplementary Figure 1. Hierarchical exposure definition

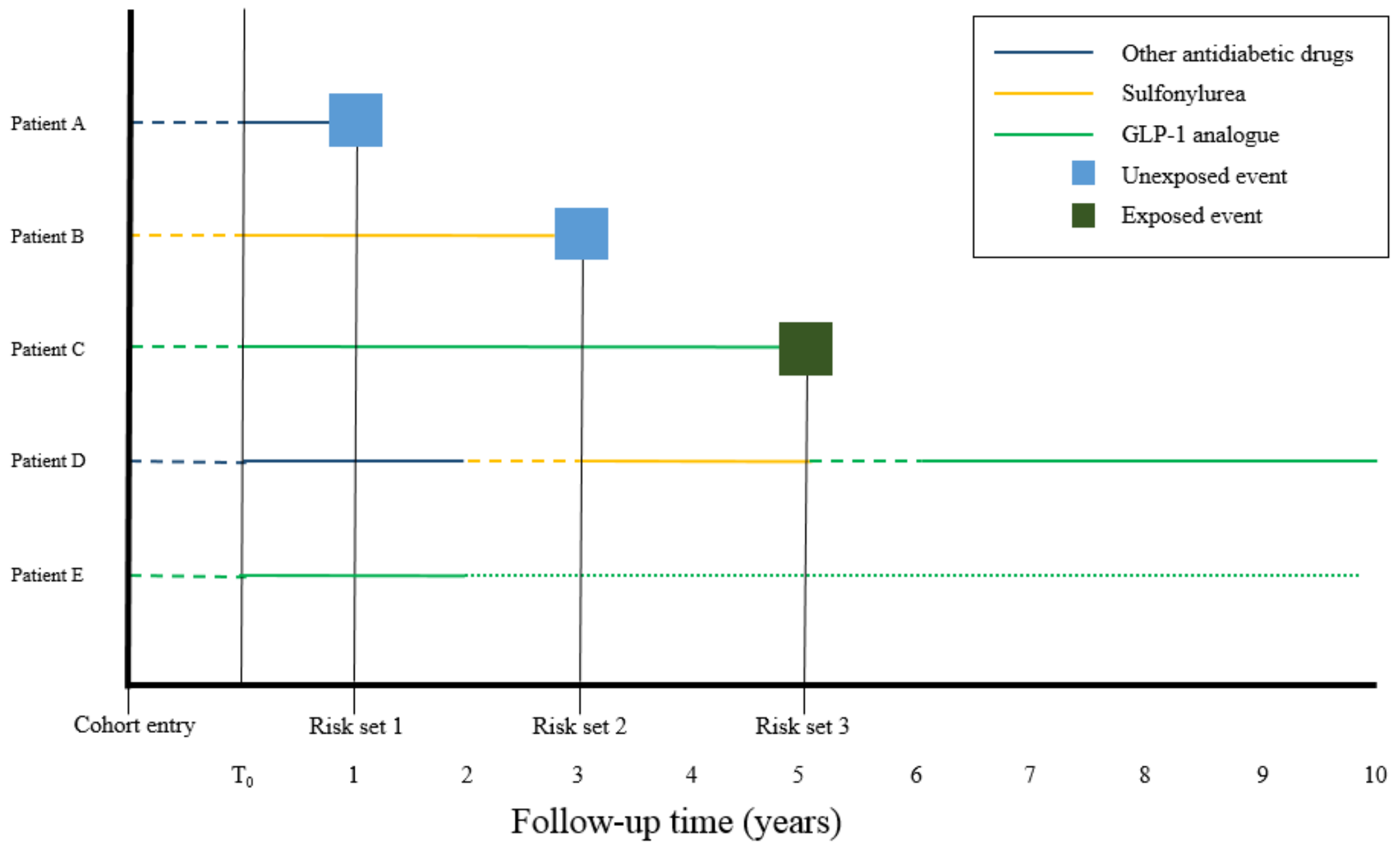


Supplementary Figure 2. Forest plot of primary and sensitivity analyses

Analysis

Primary analysis

GLP-1 analogues

DPP-4 inhibitors

2-year lag period

GLP-1 analogues

DPP-4 inhibitors

No lag period

GLP-1 analogues

DPP-4 inhibitors

Previous colorectal cancer screening

GLP-1 analogues

DPP-4 inhibitors

Disease risk score

GLP-1 analogues

DPP-4 inhibitors

Marginal structural model

GLP-1 analogues

DPP-4 inhibitors

Multiple imputation

GLP-1 analogues

DPP-4 inhibitors

Excluding in situ colorectal cancer

GLP-1 analogues

DPP-4 inhibitors
HR $\quad(95 \% \mathrm{Cl})$

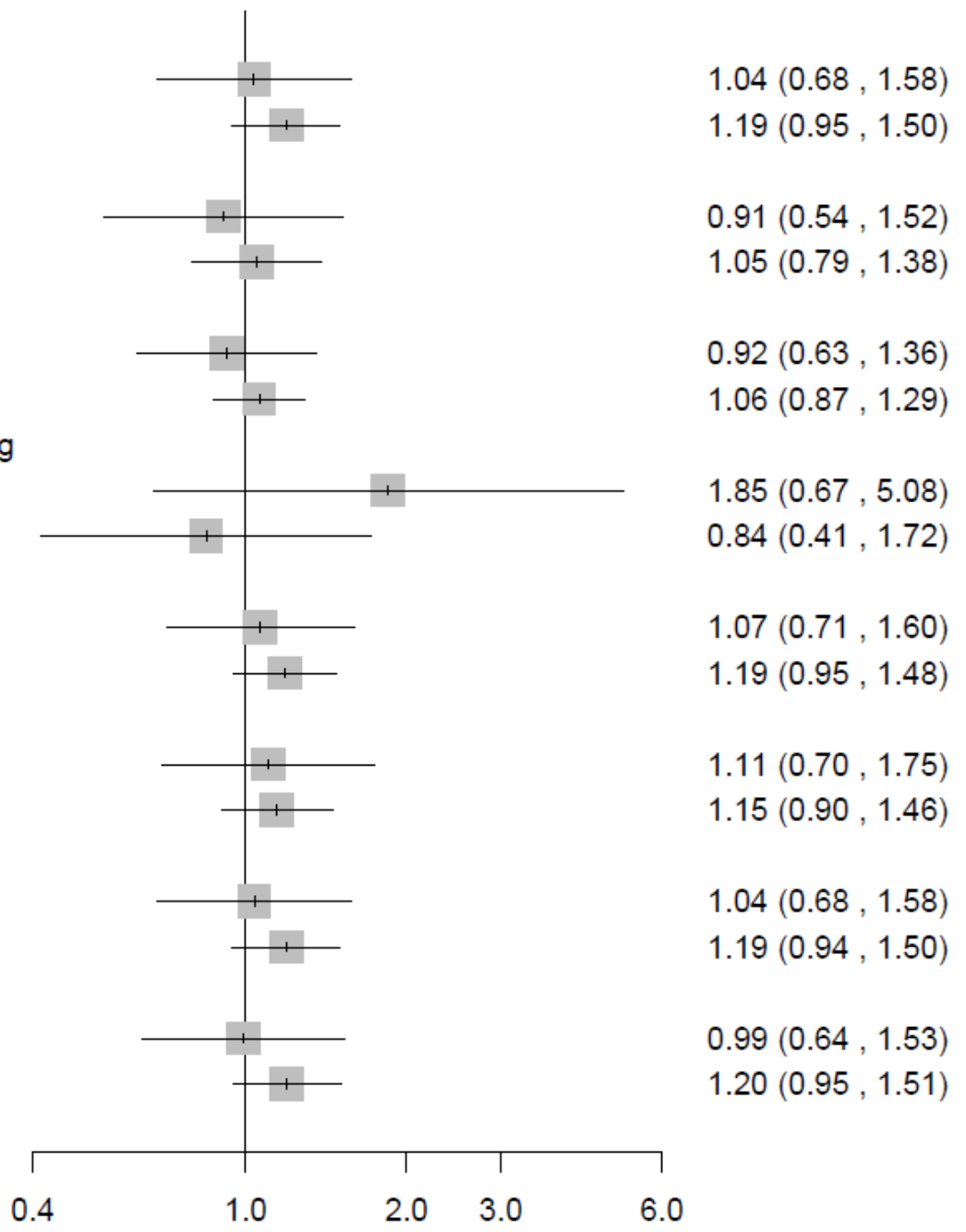




\begin{tabular}{|c|c|c|c|c|c|}
\hline Exposure $^{a}$ & Events & Person-years & $\begin{array}{l}\text { Incidence rate }{ }^{b} \\
(95 \% \mathrm{Cl})\end{array}$ & Crude HR & $\begin{array}{l}\text { Adjusted HR (95\% } \\
\mathrm{Cl})^{c}\end{array}$ \\
\hline Sulfonylureas & 302 & 151,949 & $1.99(1.77,2.22)$ & 1.00 & 1.00 [Reference] \\
\hline Exenatide only & 12 & 7222 & $1.66(0.86,2.90)$ & 0.82 & $1.03(0.57,1.87)$ \\
\hline Liraglutide only & 5 & 6349 & $0.79(0.26,1.84)$ & 0.38 & $0.54(0.22,1.32)$ \\
\hline Other GLP-1 analogues & 9 & 2563 & $3.51(1.61,6.67)$ & 1.66 & $2.25(1.14,4.43)$ \\
\hline
\end{tabular}

Abbreviations: $\mathrm{HR}$, hazard ratio; $\mathrm{Cl}$, confidence interval; GLP-1, glucagon-like peptide-1

${ }^{\text {a }}$ Use of other anti-diabetic agents types is considered in the model, but not presented in the table.

${ }^{b}$ Per 1000 Person-Years.

${ }^{\mathrm{C}}$ Adjusted for age, sex, year of cohort entry, body mass index (BMI), smoking, alcohol-related disorders (including for example alcoholism, alcoholic cirrhosis of the liver, alcoholic hepatitis and hepatic flexure), hemoglobin A1c, duration of treated diabetes, previous cancer, presence of inflammatory bowel disease, Charlson comorbidity score, neuropathy, renal disease, retinopathy, peripheral arteriopathy, aspirin, statins, the number of unique antidiabetic drugs and the total number of unique non-diabetic drugs in the year before cohort entry. 
Supplementary Table 2. Crude and Adjusted HRs for the Association Between the Use of DPP-4 Inhibitors and the Risk of Colorectal Cancer (DPP-4 Type)

\begin{tabular}{|c|c|c|c|c|c|}
\hline Exposure $^{a}$ & Events & Person-years & $\begin{array}{l}\text { Incidence rate }{ }^{b} \\
(95 \% \mathrm{Cl})\end{array}$ & Crude HR & $\begin{array}{l}\text { Adjusted HR (95\% } \\
\text { Cl) }{ }^{c}\end{array}$ \\
\hline Sulfonylureas & 241 & 127,443 & $1.89(1.66,2.15)$ & 1.00 & 1.00 [Reference] \\
\hline Sitagliptin only & 90 & 43,224 & $2.08(1.67,2.56)$ & 1.07 & $1.22(0.95,1.57)$ \\
\hline Saxagliptin only & 13 & 5276 & $2.46(1.31,4.21)$ & 1.27 & $1.38(0.78,2.42)$ \\
\hline Other DPP-4 inhibitors & 14 & 8113 & $1.73(0.94,2.90)$ & 0.88 & $0.93(0.54,1.60)$ \\
\hline
\end{tabular}

Abbreviations: $\mathrm{HR}$, hazard ratio; $\mathrm{Cl}$, confidence interval; DPP-4, dipeptidyl peptidase-4

${ }^{a}$ Use of other anti-diabetic agents types is considered in the model, but not presented in the table.

${ }^{b}$ Per 1000 Person-Years.

${ }^{\mathrm{C}}$ Adjusted for age, sex, year of cohort entry, body mass index (BMI), smoking, alcohol-related disorders (including for example alcoholism, alcoholic cirrhosis of the liver, alcoholic hepatitis and hepatic flexure), hemoglobin A1c, duration of treated diabetes, previous cancer, presence of inflammatory bowel disease, Charlson comorbidity score, neuropathy, renal disease, retinopathy, peripheral arteriopathy, aspirin, statins, the number of unique antidiabetic drugs and the total number of unique non-diabetic drugs in the year before cohort entry. 


\begin{tabular}{|c|c|c|c|c|c|}
\hline Exposure $^{a}$ & Events & Person-years & $\begin{array}{l}\text { Incidence rate }{ }^{b} \\
(95 \% \mathrm{Cl})\end{array}$ & Crude HR & $\begin{array}{l}\text { Adjusted HR (95\% } \\
\mathrm{Cl}^{c}\end{array}$ \\
\hline \multicolumn{6}{|l|}{ Colon cancer } \\
\hline Sulfonylureas & 224 & 151,949 & $1.47(1.29,1.68)$ & 1.00 & 1.00 [Reference] \\
\hline GLP-1 analogues & 22 & 16,135 & $1.36(0.85,2.06)$ & 0.89 & $1.14(0.72,1.81)$ \\
\hline \multicolumn{6}{|l|}{ Rectal cancer } \\
\hline Sulfonylureas & 78 & 151,949 & $0.51(0.41,0.64)$ & 1.00 & 1.00 [Reference] \\
\hline GLP-1 analogues & $S^{d}$ & $S^{d}$ & $0.25(0.07,0.63)$ & 0.48 & $0.71(0.25,1.99)$ \\
\hline
\end{tabular}

Abbreviations: $\mathrm{HR}$, hazard ratio; $\mathrm{Cl}$, confidence interval; GLP-1, glucagon-like peptide-1

${ }^{a}$ Use of other anti-diabetic agents types is considered in the model, but not presented in the table.

${ }^{b}$ Per 1000 Person-Years.

${ }^{\mathrm{C}}$ Adjusted for age, sex, year of cohort entry, body mass index (BMI), smoking, alcohol-related disorders (including for example alcoholism, alcoholic cirrhosis of the liver, alcoholic hepatitis and hepatic flexure), hemoglobin A1c, duration of treated diabetes, previous cancer, presence of inflammatory bowel disease, Charlson comorbidity score, neuropathy, renal disease, retinopathy, peripheral arteriopathy, aspirin, statins, the number of unique antidiabetic drugs and the total number of unique non-diabetic drugs in the year before cohort entry.

${ }^{\mathrm{d}}$ Numbers less than 5 are not displayed, as per the confidentiality policies of the Clinical Practice Research Datalink. 


\begin{tabular}{|c|c|c|c|c|c|}
\hline \multicolumn{6}{|c|}{$\begin{array}{l}\text { Supplementary Table 4. Crude and Adjusted HRs for the Associa } \\
\text { Colorectal Cancer (Colon Cancer and Rectal Cancer Specifically) }\end{array}$} \\
\hline Exposure a & Events & Person-years & $\begin{array}{l}\text { Incidence rate }{ }^{b} \\
(95 \% \mathrm{Cl})\end{array}$ & Crude HR & $\begin{array}{l}\text { Adjusted HR (95\% } \\
\mathrm{Cl})^{c}\end{array}$ \\
\hline \multicolumn{6}{|l|}{ Colon cancer } \\
\hline Sulfonylureas & 177 & 127,443 & $1.4(1.2,1.6)$ & 1.00 & 1.00 [Reference] \\
\hline DPP-4 inhibitors & 87 & 56,613 & $1.5(1.2,1.9)$ & 1.07 & $1.15(0.88,1.51)$ \\
\hline \multicolumn{6}{|l|}{ Rectal cancer } \\
\hline Sulfonylureas & 64 & 127,443 & $0.5(0.4,0.6)$ & 1.00 & 1.00 [Reference] \\
\hline DPP-4 inhibitors & 30 & 56,613 & $0.5(0.4,0.8)$ & 1.05 & $1.31(0.83,2.05)$ \\
\hline
\end{tabular}

Abbreviations: $\mathrm{HR}$, hazard ratio; $\mathrm{Cl}$, confidence interval; DPP-4, dipeptidyl peptidase-4

a Use of other anti-diabetic agents types is considered in the model, but not presented in the table.

${ }^{b}$ Per 1000 Person-Years.

${ }^{\mathrm{c}}$ Adjusted for age, sex, year of cohort entry, body mass index (BMI), smoking, alcohol-related disorders (including for example alcoholism, alcoholic cirrhosis of the liver, alcoholic hepatitis and hepatic flexure), hemoglobin A1c, duration of treated diabetes, previous cancer, presence of inflammatory bowel disease, Charlson comorbidity score, neuropathy, renal disease, retinopathy, peripheral arteriopathy, aspirin, statins, the number of unique antidiabetic drugs and the total number of unique non-diabetic drugs in the year before cohort entry. 


\begin{tabular}{|c|c|c|c|c|c|}
\hline Exposure $^{a}$ & Events & Person-years & $\begin{array}{l}\text { Incidence rate }{ }^{b} \\
(95 \% \mathrm{Cl})\end{array}$ & Crude HR & $\begin{array}{l}\text { Adjusted HR (95\% } \\
\text { Cl) }{ }^{c}\end{array}$ \\
\hline Sulfonylureas & 232 & 111,228 & $2.09(1.83,2.37)$ & 1.00 & 1.00 [Reference] \\
\hline GLP-1 analogues & 17 & 10,958 & $1.55(0.90,2.48)$ & 0.73 & $0.91(0.54,1.52)$ \\
\hline
\end{tabular}

Abbreviations: $\mathrm{HR}$, hazard ratio; $\mathrm{Cl}$, confidence interval; GLP-1, glucagon-like peptide-1

a Use of other anti-diabetic agents types is considered in the model, but not presented in the table.

b Per 1000 Person-Years.

${ }^{\mathrm{c}}$ Adjusted for age, sex, year of cohort entry, body mass index (BMI), smoking, alcohol-related disorders (including for example alcoholism, alcoholic cirrhosis of the liver, alcoholic hepatitis and hepatic flexure), hemoglobin A1c, duration of treated diabetes, previous cancer, presence of inflammatory bowel disease, Charlson comorbidity score, neuropathy, renal disease, retinopathy, peripheral arteriopathy, aspirin, statins, the number of unique antidiabetic drugs and the total number of unique non-diabetic drugs in the year before cohort entry. 
Supplementary Table 6. Crude and Adjusted HRs for the Association Between the Use of DPP-4 Inhibitors and the Risk of Colorectal Cancer (2-year Lag Period)

\begin{tabular}{|c|c|c|c|c|c|}
\hline Exposure $^{a}$ & Events & Person-years & $\begin{array}{l}\text { Incidence rate }{ }^{b} \\
(95 \% \mathrm{Cl})\end{array}$ & Crude HR & $\begin{array}{l}\text { Adjusted HR (95\% } \\
\mathrm{Cl})^{c}\end{array}$ \\
\hline Sulfonylureas & 197 & 95,803 & $2.06(1.78,2.36)$ & 1.00 & 1.00 [Reference] \\
\hline DPP-4 inhibitors & 74 & 36,988 & $2.00(1.57,2.51)$ & 0.95 & $1.05(0.79,1.38)$ \\
\hline
\end{tabular}

Abbreviations: $\mathrm{HR}$, hazard ratio; $\mathrm{Cl}$, confidence interval; DPP-4, dipeptidyl peptidase-4

a Use of other anti-diabetic agents types is considered in the model, but not presented in the table.

${ }^{b}$ Per 1000 Person-Years.

${ }^{\mathrm{C}}$ Adjusted for age, sex, year of cohort entry, body mass index (BMI), smoking, alcohol-related disorders (including for example alcoholism, alcoholic cirrhosis of the liver, alcoholic hepatitis and hepatic flexure), hemoglobin A1c, duration of treated diabetes, previous cancer, presence of inflammatory bowel disease, Charlson comorbidity score, neuropathy, renal disease, retinopathy, peripheral arteriopathy, aspirin, statins, the number of unique antidiabetic drugs and the total number of unique non-diabetic drugs in the year before cohort entry. 
Supplementary Table 7. Crude and Adjusted HRs for the Association Between the Use of GLP-1 Analogues and the Risk of Colorectal Cancer (No Lag Period)

\begin{tabular}{|c|c|c|c|c|c|}
\hline Exposure $^{a}$ & Events & Person-years & $\begin{array}{l}\text { Incidence rate }{ }^{b} \\
(95 \% \mathrm{Cl})\end{array}$ & Crude HR & $\begin{array}{l}\text { Adjusted HR (95\% } \\
\mathrm{Cl})^{c}\end{array}$ \\
\hline Sulfonylureas & 423 & 200,580 & $2.11(1.91,2.32)$ & 1.00 & 1.00 [Reference] \\
\hline GLP-1 analogues & 30 & 22,411 & $1.34(0.90,1.91)$ & 0.62 & $0.92(0.63,1.36)$ \\
\hline
\end{tabular}

Abbreviations: $\mathrm{HR}$, hazard ratio; $\mathrm{Cl}$, confidence interval; GLP-1, glucagon-like peptide-1

a Use of other anti-diabetic agents types is considered in the model, but not presented in the table.

${ }^{b}$ Per 1000 Person-Years.

${ }^{\mathrm{C}}$ Adjusted for age, sex, year of cohort entry, body mass index (BMI), smoking, alcohol-related disorders (including for example alcoholism, alcoholic cirrhosis of the liver, alcoholic hepatitis and hepatic flexure), hemoglobin A1c, duration of treated diabetes, previous cancer, presence of inflammatory bowel disease, Charlson comorbidity score, neuropathy, renal disease, retinopathy, peripheral arteriopathy, aspirin, statins, the number of unique antidiabetic drugs and the total number of unique non-diabetic drugs in the year before cohort entry. 
Supplementary Table 8. Crude and Adjusted HRs for the Association Between the Use of DPP-4 Inhibitors and the Risk of Colorectal Cancer (No Lag Period)

\begin{tabular}{|c|c|c|c|c|c|}
\hline Exposure $^{a}$ & Events & Person-years & $\begin{array}{l}\text { Incidence rate }{ }^{b} \\
(95 \% \mathrm{Cl})\end{array}$ & Crude HR & $\begin{array}{l}\text { Adjusted HR (95\% } \\
\mathrm{Cl})^{c}\end{array}$ \\
\hline Sulfonylureas & 339 & 164,135 & $2.07(1.85,2.30)$ & 1.00 & 1.00 [Reference] \\
\hline DPP-4 inhibitors & 156 & 81,792 & $1.91(1.62,2.23)$ & 0.91 & $1.06(0.87,1.29)$ \\
\hline
\end{tabular}

Abbreviations: $\mathrm{HR}$, hazard ratio; $\mathrm{Cl}$, confidence interval; DPP-4, dipeptidyl peptidase-4

a Use of other anti-diabetic agents types is considered in the model, but not presented in the table.

${ }^{b}$ Per 1000 Person-Years.

${ }^{\mathrm{C}}$ Adjusted for age, sex, year of cohort entry, body mass index (BMI), smoking, alcohol-related disorders (including for example alcoholism, alcoholic cirrhosis of the liver, alcoholic hepatitis and hepatic flexure), hemoglobin A1c, duration of treated diabetes, previous cancer, presence of inflammatory bowel disease, Charlson comorbidity score, neuropathy, renal disease, retinopathy, peripheral arteriopathy, aspirin, statins, the number of unique antidiabetic drugs and the total number of unique non-diabetic drugs in the year before cohort entry. 
Supplementary Table 9. Crude and Adjusted HRs for the Association Between the Use of GLP-1 Analogues and the Risk of Colorectal Cancer (Stratified by Screening)

\begin{tabular}{|c|c|c|c|c|c|}
\hline Exposure $^{a}$ & Events & Person-years & $\begin{array}{l}\text { Incidence rate }{ }^{b} \\
(95 \% \mathrm{Cl})\end{array}$ & Crude HR & $\begin{array}{l}\text { Adjusted HR (95\% } \\
\mathrm{Cl})^{c}\end{array}$ \\
\hline \multicolumn{6}{|l|}{ With screening } \\
\hline Sulfonylureas & 32 & 16,565 & $1.93(1.32,2.73)$ & 1.00 & 1.00 [Reference] \\
\hline GLP-1 analogues & 5 & 1844 & $2.71(0.88,6.33)$ & 1.26 & $1.85(0.67,5.08)$ \\
\hline \multicolumn{6}{|l|}{ Without screening } \\
\hline Sulfonylureas & 270 & 135,384 & $1.99(1.76,2.25)$ & 1.00 & 1.00 [Reference] \\
\hline GLP-1 analogues & 21 & 14,291 & $1.47(0.91,2.25)$ & 0.72 & $0.93(0.59,1.49)$ \\
\hline
\end{tabular}

Abbreviations: $\mathrm{HR}$, hazard ratio; $\mathrm{Cl}$, confidence interval; GLP-1, glucagon-like peptide-1

a Use of other anti-diabetic agents types is considered in the model, but not presented in the table.

${ }^{b}$ Per 1000 Person-Years.

${ }^{\mathrm{c}}$ Adjusted for age, sex, year of cohort entry, body mass index (BMI), smoking, alcohol-related disorders (including for example alcoholism, alcoholic cirrhosis of the liver, alcoholic hepatitis and hepatic flexure), hemoglobin A1c, duration of treated diabetes, previous cancer, presence of inflammatory bowel disease, Charlson comorbidity score, neuropathy, renal disease, retinopathy, peripheral arteriopathy, aspirin, statins, the number of unique antidiabetic drugs and the total number of unique non-diabetic drugs in the year before cohort entry. 
Supplementary Table 10. Crude and Adjusted HRs for the Association Between the Use of DPP-4 Inhibitors and the Risk of Colorectal Cancer (Stratified by Screening)

\begin{tabular}{|c|c|c|c|c|c|}
\hline Exposure $^{a}$ & Events & Person-years & $\begin{array}{l}\text { Incidence rate }{ }^{b} \\
(95 \% \mathrm{Cl})\end{array}$ & Crude HR & $\begin{array}{l}\text { Adjusted HR (95\% } \\
\mathrm{Cl})^{c}\end{array}$ \\
\hline \multicolumn{6}{|l|}{ With screening } \\
\hline DPP-4 inhibitors & 12 & 6940 & $1.73(0.89,3.02)$ & 0.79 & $0.84(0.41,1.72)$ \\
\hline \multicolumn{6}{|c|}{ Without screening } \\
\hline DPP-4 inhibitors & 105 & 49,673 & $2.11(1.73,2.56)$ & 1.11 & $1.24(0.97,1.59)$ \\
\hline
\end{tabular}

Abbreviations: $\mathrm{HR}$, hazard ratio; $\mathrm{Cl}$, confidence interval; DPP-4, dipeptidyl peptidase-4

a Use of other anti-diabetic agents types is considered in the model, but not presented in the table.

b Per 1000 Person-Years.

${ }^{\mathrm{c}}$ Adjusted for age, sex, year of cohort entry, body mass index (BMI), smoking, alcohol-related disorders (including for example alcoholism, alcoholic cirrhosis of the liver, alcoholic hepatitis and hepatic flexure), hemoglobin A1c, duration of treated diabetes, previous cancer, presence of inflammatory bowel disease, Charlson comorbidity score, neuropathy, renal disease, retinopathy, peripheral arteriopathy, aspirin, statins, the number of unique antidiabetic drugs and the total number of unique non-diabetic drugs in the year before cohort entry. 
Supplementary Table 11. Crude and Adjusted HRs for the Association Between the Use of GLP-1 Analogues and the Risk of Malignant Colorectal Cancer

\begin{tabular}{|c|c|c|c|c|c|}
\hline Exposure $^{a}$ & Events & Person-years & $\begin{array}{l}\text { Incidence rate }{ }^{b} \\
(95 \% \mathrm{Cl})\end{array}$ & Crude HR & $\begin{array}{l}\text { Adjusted HR (95\% } \\
\mathrm{Cl})^{c}\end{array}$ \\
\hline Sulfonylurea & 292 & 151,949 & $1.92(1.71,2.16)$ & 1.00 & 1.00 [Reference] \\
\hline
\end{tabular}

Abbreviations: $\mathrm{HR}$, hazard ratio; $\mathrm{Cl}$, confidence interval; GLP-1, glucagon-like peptide-1

a Use of other anti-diabetic agents types is considered in the model, but not presented in the table.

b Per 1000 Person-Months.

${ }^{\mathrm{c}}$ Adjusted for age, sex, year of cohort entry, body mass index (BMI), smoking, alcohol-related disorders (including for example alcoholism, alcoholic cirrhosis of the liver, alcoholic hepatitis and hepatic flexure), hemoglobin A1c, duration of treated diabetes, previous cancer, presence of inflammatory bowel disease, Charlson comorbidity score, neuropathy, renal disease, retinopathy, peripheral arteriopathy, aspirin, statins, the number of unique antidiabetic drugs and the total number of unique non-diabetic drugs in the year before cohort entry. 
Supplementary Table 12. Crude and Adjusted HRs for the Association Between the Use of DPP-4 Inhibitors and the Risk of Malignant Colorectal Cancer

\begin{tabular}{|c|c|c|c|c|c|}
\hline Exposure $^{a}$ & Events & Person-years & $\begin{array}{l}\text { Incidence rate }{ }^{b} \\
(95 \% \mathrm{CI})\end{array}$ & Crude HR & $\begin{array}{l}\text { Adjusted HR (95\% } \\
\mathrm{Cl})^{c}\end{array}$ \\
\hline Sulfonylurea & 234 & 127,443 & $1.84(1.61,2.09)$ & 1.00 & 1.00 [Reference] \\
\hline
\end{tabular}

Abbreviations: $\mathrm{HR}$, hazard ratio; $\mathrm{Cl}$, confidence interval; DPP-4, dipeptidyl peptidase-4

a Use of other anti-diabetic agents types is considered in the model, but not presented in the table.

b Per 1000 Person-Months.

${ }^{\mathrm{C}}$ Adjusted for age, sex, year of cohort entry, body mass index (BMI), smoking, alcohol-related disorders (including for example alcoholism, alcoholic cirrhosis of the liver, alcoholic hepatitis and hepatic flexure), hemoglobin A1c, duration of treated diabetes, previous cancer, presence of inflammatory bowel disease, Charlson comorbidity score, neuropathy, renal disease, retinopathy, peripheral arteriopathy, aspirin, statins, the number of unique antidiabetic drugs and the total number of unique non-diabetic drugs in the year before cohort entry. 
Supplementary Table 13. Crude and Adjusted HRs for the Association Between the Use of GLP-1 Analogues and the Risk of Colorectal Cancer (Stratified by Disease Risk Score)

\begin{tabular}{|c|c|c|c|c|c|}
\hline Exposure $^{a}$ & Events & Person-years & $\begin{array}{l}\text { Incidence rate }{ }^{b} \\
(95 \% \mathrm{Cl})\end{array}$ & Crude HR & $\begin{array}{l}\text { Adjusted HR (95\% } \\
\mathrm{Cl})^{c}\end{array}$ \\
\hline Sulfonylureas & 302 & 151,949 & $1.99(1.77,2.22)$ & 1.00 & 1.00 [Reference] \\
\hline
\end{tabular}

Abbreviations: $\mathrm{HR}$, hazard ratio; $\mathrm{Cl}$, confidence interval; GLP-1, glucagon-like peptide-1

a Use of other anti-diabetic agents types is considered in the model, but not presented in the table.

b Per 1000 Person-Years.

' Stratified by disease risk score deciles. 
Supplementary Table 14. Crude and Adjusted HRs for the Association Between the Use of DPP-4 Inhibitors and the Risk of Colorectal Cancer (Stratified by Disease Risk Score)

\begin{tabular}{|c|c|c|c|c|c|}
\hline Exposure $^{a}$ & Events & Person-years & $\begin{array}{l}\text { Incidence rate }{ }^{b} \\
(95 \% \mathrm{CI})\end{array}$ & Crude HR & $\begin{array}{l}\text { Adjusted HR (95\% } \\
\mathrm{Cl})^{c}\end{array}$ \\
\hline Sulfonylureas & 241 & 127,443 & $1.89(1.66,2.15)$ & 1.00 & 1.00 [Reference] \\
\hline
\end{tabular}

Abbreviations: $\mathrm{HR}$, hazard ratio; $\mathrm{Cl}$, confidence interval; DPP-4, dipeptidyl peptidase-4

a Use of other anti-diabetic agents types is considered in the model, but not presented in the table.

b Per 1000 Person-Years.

'Stratified by disease risk score deciles. 
Supplementary Table 15. Crude and Adjusted HRs for the Association Between the Use of GLP-1 Analogues and the Risk of Colorectal Cancer (Marginal Structure Model)

\begin{tabular}{llllll} 
Exposure $^{\text {a }}$ & Events & Person-months & $\begin{array}{l}\text { Incidence rate } \\
\mathbf{( 9 5 \%} \mathbf{C l})\end{array}$ & $\begin{array}{l}\text { Crude HR in } \\
\text { weighted model }\end{array}$ & $\begin{array}{l}\text { Adjusted HR in weighted } \\
\text { model(95\% Cl) }\end{array}$ \\
\hline Sulfonylureas & 303 & $1,882,171$ & $0.16(0.14,0.18)$ & 1.00 & $1.00[$ Reference] \\
GLP-1 analogues & 26 & 201,203 & $0.13(0.08,0.19)$ & 0.82 & $1.11(0.70,1.75)$ \\
\hline
\end{tabular}

Abbreviations: $\mathrm{HR}$, hazard ratio; $\mathrm{Cl}$, confidence interval; GLP-1, glucagon-like peptide-1

${ }^{a}$ Use of other anti-diabetic agents types is considered in the model, but not presented in the table.

b Per 1000 Person-Months.

${ }^{\mathrm{c}}$ Adjusted for age, sex, year of cohort entry, body mass index (BMI), smoking, alcohol-related disorders (including for example alcoholism, alcoholic cirrhosis of the liver, alcoholic hepatitis and hepatic flexure), hemoglobin A1c, duration of treated diabetes, previous cancer, presence of inflammatory bowel disease, Charlson comorbidity score, neuropathy, renal disease, retinopathy, peripheral arteriopathy, aspirin, statins, the number of unique antidiabetic drugs and the total number of unique non-diabetic drugs in the year before cohort entry. 
Supplementary Table 16. Crude and Adjusted HRs for the Association Between the Use of DPP-4 Inhibitors and the Risk of Colorectal Cancer (Marginal Structural Model)

\begin{tabular}{llllll} 
Exposure $^{\text {a }}$ & Events & Person-months & $\begin{array}{l}\text { Incidence rate }{ }^{\mathbf{b}} \\
\mathbf{( 9 5 \% ~ C l )}\end{array}$ & $\begin{array}{l}\text { Crude HR in } \\
\text { weighted model }\end{array}$ & $\begin{array}{l}\text { Adjusted HR in weighted } \\
\text { model(95\% CI) }\end{array}$ \\
\hline Sulfonylureas & 242 & $1,574,255$ & $0.15(0.13,0.17)$ & 1.00 & $1.00[$ Reference] \\
DPP-4 inhibitors & 117 & 707,188 & $0.17(0.14,0.20)$ & 1.02 & $1.15(0.90,1.46)$ \\
\hline
\end{tabular}

Abbreviations: $\mathrm{HR}$, hazard ratio; $\mathrm{Cl}$, confidence interval; DPP-4, dipeptidyl peptidase-4

a Use of other anti-diabetic agents types is considered in the model, but not presented in the table.

b Per 1000 Person-Months.

${ }^{c}$ Adjusted for age, sex, year of cohort entry, body mass index (BMI), smoking, alcohol-related disorders (including for example alcoholism, alcoholic cirrhosis of the liver, alcoholic hepatitis and hepatic flexure), hemoglobin A1c, duration of treated diabetes, previous cancer, presence of inflammatory bowel disease, Charlson comorbidity score, neuropathy, renal disease, retinopathy, peripheral arteriopathy, aspirin, statins, the number of unique antidiabetic drugs and the total number of unique non-diabetic drugs in the year before cohort entry. 
Supplementary Table 17. Crude and Adjusted HRs for the Association Between the Use of GLP-1 Analogues and the Risk of Colorectal Cancer (Multiple imputation)

\begin{tabular}{|c|c|c|c|c|c|}
\hline Exposure $^{a}$ & Events & Person-years & $\begin{array}{l}\text { Incidence rate }{ }^{b} \\
(95 \% \mathrm{Cl})\end{array}$ & Crude HR & $\begin{array}{l}\text { Adjusted HR (95\% } \\
\mathrm{Cl})^{c}\end{array}$ \\
\hline Sulfonylurea & 302 & 151,949 & $1.99(1.77,2.22)$ & 1.00 & 1.00 [Reference] \\
\hline
\end{tabular}

Abbreviations: $\mathrm{HR}$, hazard ratio; $\mathrm{Cl}$, confidence interval; GLP-1, glucagon-like peptide-1

a Use of other anti-diabetic agents types is considered in the model, but not presented in the table.

b Per 1000 Person-Months.

${ }^{\mathrm{C}}$ Adjusted for age, sex, year of cohort entry, body mass index (BMI), smoking, alcohol-related disorders (including for example alcoholism, alcoholic cirrhosis of the liver, alcoholic hepatitis and hepatic flexure), hemoglobin A1c, duration of treated diabetes, previous cancer, presence of inflammatory bowel disease, Charlson comorbidity score, neuropathy, renal disease, retinopathy, peripheral arteriopathy, aspirin, statins, the number of unique antidiabetic drugs and the total number of unique non-diabetic drugs in the year before cohort entry. 
Supplementary Table 18. Crude and Adjusted HRs for the Association Between the Use of DPP-4 Inhibitors and the Risk of Colorectal Cancer (Multiple imputation)

\begin{tabular}{|c|c|c|c|c|c|}
\hline Exposure $^{a}$ & Events & Person-years & $\begin{array}{l}\text { Incidence rate }{ }^{b} \\
(95 \% \mathrm{CI})\end{array}$ & Crude HR & $\begin{array}{l}\text { Adjusted HR (95\% } \\
\mathrm{Cl})^{c}\end{array}$ \\
\hline Sulfonylurea & 241 & 127,443 & $1.89(1.66,2.15)$ & 1.00 & 1.00 [Reference] \\
\hline
\end{tabular}

Abbreviations: $\mathrm{HR}$, hazard ratio; $\mathrm{Cl}$, confidence interval; DPP-4, dipeptidyl peptidase-4

a Use of other anti-diabetic agents types is considered in the model, but not presented in the table.

b Per 1000 Person-Months.

${ }^{\mathrm{C}}$ Adjusted for age, sex, year of cohort entry, body mass index (BMI), smoking, alcohol-related disorders (including for example alcoholism, alcoholic cirrhosis of the liver, alcoholic hepatitis and hepatic flexure), hemoglobin A1c, duration of treated diabetes, previous cancer, presence of inflammatory bowel disease, Charlson comorbidity score, neuropathy, renal disease, retinopathy, peripheral arteriopathy, aspirin, statins, the number of unique antidiabetic drugs and the total number of unique non-diabetic drugs in the year before cohort entry. 


\section{Chapter 6: General discussion}

This thesis investigated the association between the use of the incretin-based drugs and the incidence of colorectal cancer. In this study, compared with the use of sulfonylureas, the use of GLP-1 analogues and DPP-4 inhibitors was not associated with incident colorectal cancer. There was also no evidence of a duration-response relation by cumulative duration of use or time since initiation. Finally, our findings remained highly consistent across a number of sensitivity analyses meant to address possible sources of bias. To date, there has been one previous observational study on this association. ${ }^{35}$ Though this study had certain limitations, our study provides some additional reassurance into the safety of the incretin-based drugs. Thus, these drugs appear to be unassociated with colorectal cancer in the relative short-term.

The incretin-based drugs are a relatively new class of drugs; they have only been available in the UK for about 10 years. As such, their long-term safety has not been established. Pre-marketing and post-marketing RCTs on drugs are not designed to study adverse events that may occur in the long-term, such as cancer incidence. Therefore, drugs are approved without the complete knowledge of their safety profile. At the present time, roughly 10 years after market release, we are ready to start addressing important long-term safety issues of the incretin-based drugs. Eventually all new drugs will need to go through a process similar to the one outlined in this thesis.

While a well-conducted RCT would provide a definitive answer on this safety question, such a study would likely be unethical to conduct. Moreover, because of the rarity of colorectal cancer, it would require a very large sample size and relatively long follow-up, which would ultimately affect its feasibility. Thus, large population-based observational studies, such as the 
one described in this thesis, can provide critical information on the long-term effects of these drugs in the natural setting of clinical practice.

\subsection{Clinical implications}

When the incretin-based drugs entered the market, they were associated with a number of safety controversies. A major concern was that their use may be associated with acute pancreatitis and pancreatic cancer. ${ }^{27} 28$ Analysis of the FDA adverse event reporting data showed a 6-fold higher pancreatitic event rate among users of exenatide (GLP-1 analogue) and sitagliptin (DPP-4 inhibitor) and a 2.9-fold higher event rate of pancreatic cancer among users of exenatide compared to users of other antidiabetic drugs. ${ }^{59}$ However, a large populationbased case-control study on the association between the use of incretin-based drugs and pancreatic cancer did not find an increased risk. ${ }^{29}$ In this study, compared to the use of sulfonylureas, there was no association between the use of GLP-1 analogues and DPP-4 inhibitors and pancreatic cancer. ${ }^{29}$ Thus, the use of the incretin-based drugs may be unassociated with pancreatic cancer in the relative short term. In terms of other cancers, there has been an association between the use of the incretin-based drugs and breast cancer, but this association is likely a result of detection bias. ${ }^{30}$

Our study further adds to the safety profile of the incretin-based drugs, as we did not observe an association with colorectal cancer. To date, the use of the incretin-based drugs appears to be unrelated to the incidence of several cancers. Though we cannot completely rule out an increased risk as data is currently limited to 10 years, these drugs appear to be safe with respect to cancer risk in the relative short-term. 


\subsection{Future directions}

While the observed lack of association between the use of the incretin-based drugs and colorectal cancer is indicative of their safety in the short-term, it is too soon to conclusively exclude the possibility of an increased risk associated with their use. Before recommendations are given to regulatory agencies, other well conducted observational studies must be conducted to confirm our findings. Further, this association should be reassessed in the future to allow for the accrual of more follow-up time and users.

Other future directions include returning to the cohort of patients randomized in the original RCTs, and extending their follow-up to the present time. With the accrual of more person-time, long-term adverse events can be studied using this design. Though informative, this type of study would not be conclusive on its own. RCTs are limited by small sample size and highly selective patient populations, and thus analyses using these patient populations would be subject to the same limitations. Furthermore, duration of follow-up may be limited if participants discontinue the drug immediately after the RCT concludes. Even though these patients will have long follow-up, if they stop using the drug after a short trial, they may not have had sufficient exposure to reach the critical threshold for cancer incidence. Assessing cancer incidence using this patient population would be an interesting method to corroborate findings of more traditional observational studies. Population-based observational studies remain an important tool to assess the incidence of long-term adverse events using real world data and drug utilization patterns observed in clinical settings.

To conclude, these drugs will require continued surveillance and monitoring to assess their long-term safety. Because there is a plausible biological mechanism supporting an 
increased risk of cancer, ${ }^{82}$ their long-term use must be carefully monitored. Overall, this study supports the safety of the incretin-based drugs in the relative short-term, though we cannot entirely rule out an increased risk of colorectal cancer associated with their use. 


\section{Chapter 7: Conclusion}

Our study illustrated that the use of the incretin-based drugs, GLP-1 analogues and DPP4 inhibitors, was not associated with the incidence of colorectal cancer. In secondary analyses, there was no association by cumulative duration of use or time since initiation. Our study provides some reassurance to regulatory agencies, physicians and patients, as we were able to rule out an increase in the risk of colorectal cancer in the relative short-term.

This study adds important information with respect to the safety profile of incretinbased drugs. Overall, the use of these drugs appears to be unassociated with the incidence of a number of different cancers (pancreatic, breast and colorectal). Future research should investigate possible associations with other cancers, and reassess these known associations in the long-term. 


\section{Chapter 8: References}

1. Diabetes in Canada. Secondary Diabetes in Canada 2016.

2. IDF Diabetes Atlas. Secondary IDF Diabetes Atlas 2015. http://www.diabetesatlas.org/.

3. Schlienger J-L. [Type 2 diabetes complications]. Presse medicale (Paris, France : 1983) 2013;42(5):839-48.

4. Stolar M. Glycemic Control and Complications in Type 2 Diabetes Mellitus. The American Journal of Medicine;123(3):S3-S11.

5. Cannata D, Fierz Y, Vijayakumar A, et al. Type 2 Diabetes and Cancer: What Is the Connection? Mount Sinai Journal of Medicine: A Journal of Translational and Personalized Medicine 2010;77(2):197-213.

6. Larsson SC, Orsini N, Wolk A. Diabetes mellitus and risk of colorectal cancer: a meta-analysis. J Natl Cancer Inst 2005;97(22):1679-87.

7. Giovannucci E, Harlan DM, Archer MC, et al. Diabetes and Cancer: A consensus report. Diabetes Care 2010;33(7):1674-85.

8. Hu FB, Manson JE, Liu S, et al. Prospective Study of Adult Onset Diabetes Mellitus (Type 2) and Risk of Colorectal Cancer in Women. JNCl: Journal of the National Cancer Institute 1999;91(6):542-47.

9. Satija A, Spiegelman D, Giovannucci E, et al. Type 2 diabetes and risk of cancer. BMJ : British Medical Journal 2015;350.

10. Vigneri P, Frasca F, Sciacca L, et al. Diabetes and cancer. Endocr Relat Cancer 2009;16(4):1103-23. 
11. Yerrabothala S, Shaaban H, Capo G, et al. The impact of diabetes mellitus on breast cancer outcomes: a single center retrospective study. Pathol Oncol Res 2014;20(1):209-14.

12. Klil-Drori AJ, Azoulay L, Pollak MN. Cancer, obesity, diabetes, and antidiabetic drugs: is the fog clearing? Nat Rev Clin Oncol 2017;14(2):85-99.

13. Kasper JS, Giovannucci E. A meta-analysis of diabetes mellitus and the risk of prostate cancer. Cancer Epidemiol Biomarkers Prev 2006;15(11):2056-62.

14. Waters KM, Henderson BE, Stram DO, et al. Association of Diabetes With Prostate Cancer Risk in the Multiethnic Cohort. Am J Epidemiol 2009;169(8):937-45.

15. Tuffier T. Diabete et neoplasmes. Archives generales de medecine 1888;7:129-40.

16. Col NF, Ochs L, Springmann V, et al. Metformin and breast cancer risk: a meta-analysis and critical literature review. Breast Cancer Res Treat 2012;135(3):639-46.

17. DeCensi A, Puntoni M, Goodwin P, et al. Metformin and Cancer Risk in Diabetic Patients: A Systematic Review and Meta-analysis. Cancer Prevention Research 2010;3(11):1451-61.

18. Gandini S, Puntoni M, Heckman-Stoddard BM, et al. Metformin and Cancer Risk and Mortality: A Systematic Review and Meta-analysis Taking into Account Biases and Confounders. Cancer Prevention Research 2014;7(9):867-85.

19. Suissa S, Azoulay L. Metformin and the Risk of Cancer: Time-related biases in observational studies. Diabetes Care 2012;35(12):2665-73.

20. Tuccori M, Wu JW, Yin H, et al. The Use of Glyburide Compared With Other Sulfonylureas and the Risk of Cancer in Patients With Type 2 Diabetes. Diabetes Care 2015;38(11):2083-89. 
21. Yang $\mathrm{X}$, So WY, Ma RCW, et al. Use of sulphonylurea and cancer in type 2

diabetes\&\#x2014;The Hong Kong Diabetes Registry. Diabetes Res Clin Pract;90(3):34351.

22. Wu L, Zhu J, Prokop L, et al. Pharmacologic Therapy of Diabetes and Overall Cancer Risk and Mortality: A Meta-Analysis of 265 Studies. Sci Rep 2015;5:10147.

23. Bosetti C, Rosato V, Buniato D, et al. Cancer risk for patients using thiazolidinediones for type 2 diabetes: a meta-analysis. Oncologist 2013;18(2):148-56.

24. Tuccori M, Filion KB, Yin $\mathrm{H}$, et al. Pioglitazone use and risk of bladder cancer: population based cohort study. BMJ 2016;352:i1541.

25. Suissa S, Azoulay L, Dell'Aniello S, et al. Long-term effects of insulin glargine on the risk of breast cancer. Diabetologia 2011;54(9):2254.

26. Drucker DJ, Sherman SI, Gorelick FS, et al. Incretin-Based Therapies for the Treatment of Type 2 Diabetes: Evaluation of the Risks and Benefits. Diabetes Care 2010;33(2):428-33.

27. Gale EAM. GLP-1-Based Therapies and the Exocrine Pancreas: More Light, or Just More Heat? Diabetes 2012;61(5):986-88.

28. Łabuzek K, Kozłowski M, Szkudłapski D, et al. Incretin-based therapies in the treatment of type 2 diabetes - More than meets the eye? Eur J Intern Med;24(3):207-12.

29. Azoulay L, Filion KB, Platt RW, et al. Incretin based drugs and the risk of pancreatic cancer: international multicentre cohort study. BMJ 2016;352:i581.

30. Hicks BM, Yin H, Yu OHY, et al. Glucagon-like peptide-1 analogues and risk of breast cancer in women with type 2 diabetes: population based cohort study using the UK Clinical Practice Research Datalink. BMJ 2016;355. 
31. Summary Review for Regulatory Action SAXENDA (liraglutide [rDNA origin] injection)

32. Leiter L, Teoh H, Mosenzon O, et al. Frequency of cancer events with saxagliptin in the SAVOR-TIMI 53 trial. Diabetes, Obesity and Metabolism 2016;18(2):186-90.

33. Engel SS, Round E, Golm GT, et al. Safety and tolerability of sitagliptin in type 2 diabetes: pooled analysis of 25 clinical studies. Diabetes Ther 2013;4(1):119-45.

34. Liraglutide (injection) for the Treatment of Patients with Type 2 Diabetes Food and Drug Administration.

35. Htoo PT, Buse JB, Gokhale M, et al. Effect of glucagon-like peptide-1 receptor agonists and dipeptidyl peptidase-4 inhibitors on colorectal cancer incidence and its precursors. Eur J Clin Pharmacol 2016;72(8):1013-23.

36. Type 2 diabetes. Secondary Type 2 diabetes 2015. https://www.canada.ca/en/publichealth/services/diseases/type-2-diabetes.html.

37. Doucet G, Beatty M. The Cost of Diabetes in Canada: The Economic Tsunami. Canadian Journal of Diabetes;34(1):27-29.

38. Chen L, Magliano DJ, Zimmet PZ. The worldwide epidemiology of type 2 diabetes mellituspresent and future perspectives. Nat Rev Endocrinol 2012;8(4):228-36.

39. Schram MT, Baan CA, Pouwer F. Depression and Quality of Life in Patients with Diabetes: A Systematic Review from the European Depression in Diabetes (EDID) Research Consortium. Curr Diabetes Rev 2009;5(2):112-19.

40. Scheen AJ. Pathophysiology of type 2 diabetes. Acta Clin Belg 2003;58(6):335-41. 
41. Kahn SE. The relative contributions of insulin resistance and beta-cell dysfunction to the pathophysiology of Type 2 diabetes. Diabetologia 2003;46(1):3-19.

42. Polonsky KS, Burant CF. Chapter 31 - Type 2 Diabetes Mellitus. Williams Textbook of Endocrinology (Thirteenth Edition). Philadelphia: Content Repository Only!, 2016:1385450.

43. Prentki M, Nolan CJ. Islet $\beta$ cell failure in type 2 diabetes. J Clin Invest 2006;116(7):1802-12.

44. Olokoba AB, Obateru OA, Olokoba LB. Type 2 Diabetes Mellitus: A Review of Current Trends. Oman Med J 2012;27(4):269-73.

45. Cox ME, Edelman D. Tests for Screening and Diagnosis of Type 2 Diabetes. Clin Diabetes 2009;27(4):132-38.

46. Saudek CD, Brick JC. The Clinical Use of Hemoglobin A1c. Journal of diabetes science and technology (Online) 2009;3(4):629-34.

47. Chaudhury A, Duvoor C, Reddy Dendi VS, et al. Clinical Review of Antidiabetic Drugs: Implications for Type 2 Diabetes Mellitus Management. Front Endocrinol (Lausanne) 2017;8:6.

48. Hu FB, Manson JE, Stampfer MJ, et al. Diet, lifestyle, and the risk of type 2 diabetes mellitus in women. N Engl J Med 2001;345(11):790-7.

49. Mahler RJ, Adler ML. Type 2 Diabetes Mellitus: Update on Diagnosis, Pathophysiology, and Treatment. The Journal of Clinical Endocrinology \& Metabolism 1999;84(4):1165-71.

50. Lucis O. The status of metformin in Canada. Can Med Assoc J 1983;128(1):24.

51. Keegan MT. Pharmacology and Physiology for Anesthesia: Elsevier 2013:536-58. 
52. Effect of intensive blood-glucose control with metformin on complications in overweight patients with type 2 diabetes (UKPDS 34). UK Prospective Diabetes Study (UKPDS) Group. Lancet 1998;352(9131):854-65.

53. Quianzon CCL, Cheikh IE. History of current non-insulin medications for diabetes mellitus. Journal of Community Hospital Internal Medicine Perspectives 2012;2(3):10.3402/jchimp.v2i3.19081.

54. Azoulay L, Suissa S. Sulfonylureas and the risks of cardiovascular events and death: a methodological meta-regression analysis of the observational studies. Diabetes Care 2017;40(5):706-14.

55. Nissen SE, Wolski K. Effect of Rosiglitazone on the Risk of Myocardial Infarction and Death from Cardiovascular Causes. N Engl J Med 2007;356(24):2457-71.

56. Nauck MA, Vilsbøll T, Gallwitz B, et al. Incretin-Based Therapies Viewpoints on the way to consensus. Diabetes Care 2009;32(suppl 2):S223-S31.

57. Drucker DJ, Nauck MA. The incretin system: glucagon-like peptide-1 receptor agonists and dipeptidyl peptidase-4 inhibitors in type 2 diabetes. The Lancet 2006;368(9548):1696705.

58. McDougall C, McKay GA, Fisher M. Drugs for diabetes: part 5 DPP-4 inhibitors. British Journal of Cardiology 2011;18(3):130.

59. Elashoff M, Matveyenko AV, Gier B, et al. Pancreatitis, pancreatic, and thyroid cancer with glucagon-like peptide-1-based therapies. Gastroenterology 2011;141(1):150-6.

60. Mikhail N. Place of sodium-glucose co-transporter type 2 inhibitors for treatment of type 2 diabetes. World J Diabetes 2014;5(6):854-59. 
61. Rosenstock J, Ferrannini E. Euglycemic Diabetic Ketoacidosis: A Predictable, Detectable, and Preventable Safety Concern With SGLT2 Inhibitors. Diabetes Care 2015;38(9):1638-42.

62. SGLT2 inhibitors: information on potential risk of toe amputation to be included in prescribing information. European Medicines Agency.

63. FDA Drug Safety Communication: FDA confirms increased risk of leg and foot amputations with the diabetes medicine canagliflozin (Invokana, Invokamet, Invokamet XR). Secondary FDA Drug Safety Communication: FDA confirms increased risk of leg and foot amputations with the diabetes medicine canagliflozin (Invokana, Invokamet, Invokamet XR) May 16, 2017. https://www.fda.gov/Drugs/DrugSafety/ucm557507.htm.

64. Quianzon CC, Cheikh I. History of insulin. Journal of Community Hospital Internal Medicine Perspectives 2012;2(2):10.3402/jchimp.v2i2.18701.

65. Swinnen SG, Hoekstra JB, DeVries JH. Insulin Therapy for Type 2 Diabetes. Diabetes Care 2009;32(Suppl 2):S253-S59.

66. Draznin B. Mechanism of the mitogenic influence of hyperinsulinemia. Diabetol Metab Syndr 2011;3(1):10.

67. Papa V, Pezzino V, Costantino A, et al. Elevated insulin receptor content in human breast cancer. J Clin Invest 1990;86(5):1503-10.

68. Weber MM, Fottner C, Liu SB, et al. Overexpression of the insulin-like growth factor I receptor in human colon carcinomas. Cancer 2002;95(10):2086-95.

69. Tsilidis KK, Kasimis JC, Lopez DS, et al. Type 2 diabetes and cancer: umbrella review of metaanalyses of observational studies. BMJ : British Medical Journal 2015;350. 
70. Canadian Cancer Society's Advisory Committee on Cancer Statistics. Secondary Canadian Cancer Society's Advisory Committee on Cancer Statistics 2016. http://www.cancer.ca/en/cancer-information/cancertype/colorectal/statistics/?region=qc.

71. Haggar FA, Boushey RP. Colorectal Cancer Epidemiology: Incidence, Mortality, Survival, and Risk Factors. Clin Colon Rectal Surg 2009;22(4):191-97.

72. Boyle P, Leon ME. Epidemiology of colorectal cancer. Br Med Bull 2002;64(1):1-25.

73. Zauber AG. The Impact of Screening on Colorectal Cancer Mortality and Incidence - Has It Really Made a Difference? Dig Dis Sci 2015;60(3):681-91.

74. Arnold M, Sierra MS, Laversanne M, et al. Global patterns and trends in colorectal cancer incidence and mortality. Gut 2016.

75. Maroun J, Ng E, Berthelot JM, et al. Lifetime costs of colon and rectal cancer management in Canada. Chronic Dis Can 2003;24(4):91-101.

76. Lynch H, Lynch P, Lanspa S, et al. Review of the Lynch syndrome: history, molecular genetics, screening, differential diagnosis, and medicolegal ramifications. Clin Genet 2009;76(1):1-18.

77. de Kort S, Masclee AAM, Sanduleanu S, et al. Higher risk of colorectal cancer in patients with newly diagnosed diabetes mellitus before the age of colorectal cancer screening initiation. Sci Rep 2017;7:46527.

78. Smiechowski B, Azoulay L, Yin H, et al. The Use of Metformin and Colorectal Cancer Incidence in Patients with Type II Diabetes Mellitus. Cancer Epidemiology Biomarkers \&amp; Prevention 2013;22(10):1877-83. 
79. Gupta V. Glucagon-like peptide-1 analogues: An overview. Indian J Endocrinol Metab 2013;17(3):413-21.

80. Pathak R, Bridgeman MB. Dipeptidyl Peptidase-4 (DPP-4) Inhibitors In the Management of Diabetes. Pharmacy and Therapeutics 2010;35(9):509-13.

81. Halfdanarson TR, Pannala R. Incretins and risk of neoplasia. BMJ : British Medical Journal 2013;346.

82. Koehler JA, Baggio LL, Yusta B, et al. GLP-1R agonists promote normal and neoplastic intestinal growth through mechanisms requiring Fgf7. Cell Metab 2015;21(3):379-91.

83. Koehler JA, Kain T, Drucker DJ. Glucagon-like peptide-1 receptor activation inhibits growth and augments apoptosis in murine CT26 colon cancer cells. Endocrinology 2011;152(9):3362-72.

84. Tawfik MK, Mohamed MI. Exenatide suppresses 1,2-dimethylhydrazine-induced colon cancer in diabetic mice: Effect on tumor angiogenesis and cell proliferation. Biomed Pharmacother 2016;82:106-16.

85. Stulc T, Sedo A. Inhibition of multifunctional dipeptidyl peptidase-IV: Is there a risk of oncological and immunological adverse effects? Diabetes Res Clin Pract 2010;88(2):12531.

86. Masur K, Schwartz F, Entschladen F, et al. DPPIV inhibitors extend GLP-2 mediated tumour promoting effects on intestinal cancer cells. Regul Pept 2006;137(3):147-55.

87. Amritha C, KumArAvelu P, ChellAthAi DD. Evaluation of Anti Cancer Effects of DPP-4 Inhibitors in Colon Cancer-An Invitro Study. Journal of clinical and diagnostic research: JCDR 2015;9(12):FC14. 
88. Femia AP, Raimondi L, Maglieri G, et al. Long-term treatment with Sitagliptin, a dipeptidyl peptidase-4 inhibitor, reduces colon carcinogenesis and reactive oxygen species in 1,2dimethylhydrazine-induced rats. Int J Cancer 2013;133(10):2498-503.

89. Santos AM, Jung J, Aziz N, et al. Targeting fibroblast activation protein inhibits tumor stromagenesis and growth in mice. The Journal of Clinical Investigation 2009;119(12):3613-25.

90. Assessment Report for Victoza. European Medicines Agency.

91. Assessment report: Saxenda. European Medicines Agency.

92. Pfeffer MA, Claggett B, Diaz R, et al. Lixisenatide in Patients with Type 2 Diabetes and Acute Coronary Syndrome. N Engl J Med 2015;373(23):2247-57.

93. Marso SP, Bain SC, Consoli A, et al. Semaglutide and Cardiovascular Outcomes in Patients with Type 2 Diabetes. N Engl J Med 2016;375(19):1834-44.

94. Marso SP, Daniels GH, Brown-Frandsen K, et al. Liraglutide and cardiovascular outcomes in type 2 diabetes. N Engl J Med 2016;375(4):311-22.

95. Pi-Sunyer X, Astrup A, Fujioka K, et al. A randomized, controlled trial of $3.0 \mathrm{mg}$ of liraglutide in weight management. N Engl J Med 2015;373(1):11-22.

96. White WB, Cannon CP, Heller SR, et al. Alogliptin after acute coronary syndrome in patients with type 2 diabetes. N Engl J Med 2013;369(14):1327-35.

97. Green JB, Bethel MA, Armstrong PW, et al. Effect of sitagliptin on cardiovascular outcomes in type 2 diabetes. N Engl J Med 2015;373(3):232-42.

98. Herrett E, Gallagher AM, Bhaskaran K, et al. Data Resource Profile: Clinical Practice Research Datalink (CPRD). Int J Epidemiol 2015;44(3):827-36. 
99. Lawrenson R, Williams T, Farmer R. Clinical information for research; the use of general practice databases. J Public Health Med 1999;21(3):299-304.

100. Jick SS, Kaye JA, Vasilakis-Scaramozza C, et al. Validity of the general practice research database. Pharmacotherapy 2003;23(5):686-9.

101. Boggon R, Staa TP, Chapman M, et al. Cancer recording and mortality in the General Practice Research Database and linked cancer registries. Pharmacoepidemiol Drug Saf 2013;22(2):168-75.

102. Hong J-L, Meier CR, Sandler RS, et al. Risk of colorectal cancer after initiation of orlistat: matched cohort study. BMJ : British Medical Journal 2013;347.

103. Vinogradova Y, Coupland C, Hippisley-Cox J. Exposure to bisphosphonates and risk of gastrointestinal cancers: series of nested case-control studies with QResearch and CPRD data. BMJ : British Medical Journal 2013;346.

104. Tsilidis KK, Capothanassi D, Allen NE, et al. Metformin does not affect cancer risk: a cohort study in the U.K. Clinical Practice Research Datalink analyzed like an intention-to-treat trial. Diabetes Care 2014;37(9):2522-32.

105. Yang YX, Hennessy S, Lewis JD. Insulin therapy and colorectal cancer risk among type 2 diabetes mellitus patients. Gastroenterology 2004;127(4):1044-50.

106. Dregan A, Moller H, Murray-Thomas T, et al. Validity of cancer diagnosis in a primary care database compared with linked cancer registrations in England. Population-based cohort study. Cancer Epidemiol 2012;36(5):425-29. 
107. Arbogast PG, Ray WA. Performance of disease risk scores, propensity scores, and traditional multivariable outcome regression in the presence of multiple confounders. Am J Epidemiol 2011;174(5):613-20.

108. Arbogast Patrick GP. Use of disease risk scores in pharmacoepidemiologic studies. Statistical Methods in Medical Research: An International Review Journal;18(1):67-80.

109. Robins JM, Hernan MA, Brumback B. Marginal structural models and causal inference in epidemiology. Epidemiology 2000:550-60.

110. Hernán MÁ, Brumback B, Robins JM. Marginal structural models to estimate the causal effect of zidovudine on the survival of HIV-positive men. Epidemiology 2000;11(5):56170.

111. Cole SR, Hernan MA. Constructing inverse probability weights for marginal structural models. Am J Epidemiol 2008;168(6):656-64.

112. Schafer JL. Analysis of incomplete multivariate data: CRC press, 1997.

113. Rubin DB. Multiple Imputation for Nonresponse in Surveys (Wiley Series in Probability and Statistics). 1987.

114. White IR, Royston P. Imputing missing covariate values for the Cox model. Stat Med 2009;28(15):1982-98.

115. Sterne JA, White IR, Carlin JB, et al. Multiple imputation for missing data in epidemiological and clinical research: potential and pitfalls. BMJ 2009;338:b2393. 